CU-TP-979

\title{
REACTION OPERATOR APPROACH TO NON-ABELIAN ENERGY LOSS
}

\author{
M. Gyulassy ${ }^{1}$, P. Levai ${ }^{1,2}$ and I. Vitev ${ }^{1}$ \\ ${ }^{1}$ Dept. Physics, Columbia University, 538 W 120-th Street, \\ New York, NY 10027, USA \\ ${ }^{2}$ KFKI Research Institute for Particle and Nuclear Physics, \\ P.O. Box 49, Budapest, 1525, Hungary
}

June 8, 2000

\begin{abstract}
A systematic expansion of the induced inclusive gluon radiation associated with jet production in a dense QCD plasma is derived using a reaction operator formalism. Analytic expressions for the transverse momentum and light-cone momentum distributions are derived to all orders in powers of the gluon opacity of the medium, $N \sigma_{g} / A=L / \lambda_{g}$. The reaction operator approach also leads to a simple algebraic proof of the "color triviality" of single inclusive distributions and to a solvable set of recursion relations. The analytic solution generalizes previous continuum solutions (BDMPS) for applications to mesoscopic QCD plasmas. The solution is furthermore not restricted to uncorrelated geometries and allows for evolving screening scales as well as the inclusion of finite kinematic constraints. The later is particularly important because below LHC energies the kinematic constraints significantly decrease the non-abelian energy loss. Our solution for the inclusive distribution also generalizes the finite order exclusive (tagged) distribution case studied previously (GLV1). The form of the analytic solution is well suited for numerical implementation in Monte Carlo event generators to enable more accurate calculations of jet quenching in ultra-relativistic nuclear collisions. Numerical results illustrating the constributions of the first three orders in opacity are compared to the "self-quenching" hard radiation intensity. A surprising result is that the induced gluon radiation intensity is dominated by the (quadratic in $L$ ) first order opacity contribution for realistic geometries and jet energies in nuclear collisions.
\end{abstract}

PACS numbers: $12.38 . \mathrm{Mh} ; 24.85 .+\mathrm{p} ; 25.75 .-\mathrm{q}$

Keywords: Jet Quenching, Non-abelian energy loss, Opacity expansion, Reaction operator

\section{INTRODUCTION AND SUMMARY}

One of the expected signatures of the quark-gluon plasma created at RHIC and LHC energies of $\sqrt{s} \simeq$ $200 \mathrm{AGeV}$ and $\sqrt{s} \simeq 1500 \mathrm{AGeV}$ is jet quenching [ 11]-[28]. At the SPS energies of $\sqrt{s} \simeq 20 \mathrm{AGeV}$, on the other hand, no quenching of moderate $p_{\perp}<4 \mathrm{GeV}$ hadrons [24] was observed even in $P b+P b$.. This may be due to the break-down of pQCD at such low momentum scales and the difficulty of disentangling non-perturbative multiparticle production effects such as the Cronin and intrinsic $k_{T}$ effects [25]. Also finite kinematic constraints may limit the energy loss at low jet energies. In addition, at SPS energies jet propagation in high density matter is limited to at most a few $\mathrm{fm} / \mathrm{c}$ due to rapid longitudinal expansion. This 
motivated our previous work (GLV1) [26, 27] to study the problem of energy loss in "thin" plasmas. In that work we considered the exclusive (tagged) case of energy loss associated with a fixed number of interactions. Rough numerical estimates for nominal $5 \mathrm{GeV}$ jets suggested that at those relative small energies and small opacities, the radiative energy loss may indeed be much smaller than predicted by Baier, Dokshitzer, Mueller, Peigné and Schiff (BDMPS) [ 10, 11, 12, 16] and Zhakharov [13, 14] for asymptotic jet energies.

Several approaches have been advanced to compute non-abelian energy loss. One approach is aimed at treating relatively "thick" targets, which while small compared to the jet coherence length are large enough so that many collisions occur in the medium. This leads to a continuum formulation of the problem. In [ 10, 11, 12, 16] an effective 2D Schroedinger equation formulation was introduced, and in [ 13, 14, 18, 19, 20] a path integral formulation of the problem was developed. Another approach [ 6, 7, 26, 27, 28, which we extend here, aims to address the problem of radiative energy loss in "thin" plasmas, a few mean free paths thick, by computing directly the radiation pattern from the finite number of Feynman diagrams for the case of a few collisions.

The advantage of the former is that it can make direct contact with the conventional Landau-PomeranchukMigdal effect [30, 31] in QED. It has the disadvantage that the continuum solutions obtained for the case of many collisions cannot be directly applied to the case of a few collisions. Also the effects of finite kinematical constraints are difficult to include in such approaches. The advantage of the second approach is that the few collision case can be computed directly from the finite number of amplitudes involved. The disadvantage is that the LPM limit is out of reach, and the work involved in summing diagrams increases exponentially with the number of collisions. In the present paper we introduce a new approach to bridge the gap between these approaches. Our new approach is based on the construction of a suitable reaction operator, $\hat{R}_{n}$, from which recursion relations for the inclusive gluon distribution can be derived and solved analytically at arbitrary order $n$.

For "thin" QCD plasmas that can be formed in nuclear collisions, the gluon radiation intensity can be studied systematically through an expansion in powers of opacity defined by the mean number of collisions in the medium

$$
\bar{n}=\frac{L}{\lambda}=\frac{N \sigma_{e l}}{A_{\perp}}=\int d z \int d^{2} \mathbf{q} \frac{d \sigma_{e l}(z)}{d^{2} \mathbf{q}} \rho\left(z, \tau=\frac{z}{c}\right) \approx \frac{d N}{d y} \frac{\sigma_{e l}}{2 \pi R_{G}^{2}} \log \frac{R_{G}}{\tau_{0}},
$$

where $N$ is the number of targets in the medium of transverse area $A_{\perp}$. An opacity expansion in terms of the path integral formulation was introduced in [ 19, 20, 34]. In [26, 27] we considered the exclusive tagged case where all $N$ target partons interacting with the jet. Here (and [28]) we extend that calculation to the inclusive case where fluctuations of the number of collisions are allowed and only the geometry is fixed.

For a homogeneous rectangular target of thickness, $L$, the density is $\rho=N /\left(L A_{\perp}\right)$, and the mean free path is $\lambda=1 /\left(\rho \sigma_{e l}\right)$. The opacity is then simply $L / \lambda=N \sigma_{e l} / A_{\perp}$. For application to high transverse momentum jets propagating through cylindrical nuclear reaction geometries, we can interpret $L=1.2 A^{1 / 3} \equiv R_{s}$. For more realistic 3+1D Bjorken and transverse expanding Gaussian cylindrical geometry such as

$$
\rho(\overrightarrow{\mathbf{x}}, \tau)=\rho_{0}\left(\frac{\tau_{0}}{\tau}\right) \exp \left(-\frac{\overrightarrow{\mathbf{x}}^{2}+\Delta \tau^{2}}{R_{G}^{2}}\right) I_{0}\left(\frac{2|\overrightarrow{\mathbf{x}}| \Delta \tau}{R_{G}^{2}}\right)
$$

$L$ is replaced by the equivalent rms Gaussian transverse radius $R_{G}=0.75 A^{1 / 3} \mathrm{fm}$. The rightmost expression in (11) is obtained by averaging the number of collisions of a transverse jet in this expanding Gaussian cylinder with the initial jet production coordinate, $\overrightarrow{\mathbf{x}}_{0}$, averaged over $\rho\left(\overrightarrow{\mathbf{x}}_{0}, \tau_{0}\right)$. Here $\tau_{0}$ is the formation time of the plasma. At RHIC energies $(\sqrt{s} \simeq 200 \mathrm{AGeV})$ the expected rapidity density of the gluons is $d N / d y \simeq 1000$ for $A=200$ [29]. For a typical elastic gluon-gluon cross section $\sigma_{e l} \sim 2 \mathrm{mb}$ and a plasma formation time $\sim 0.5 \mathrm{fm} / \mathrm{c}$, the opacity is moderately small $\bar{n}<10$. This suggests that neither the thin nor thick plasma approximations may apply for applications to nuclear collisions. Therefore, an alternate method is needed to handle the intermediate (mesoscopic) case.

An important simplification may be anticipated due the non-abelian analog [ 6, 10, 28] of the LandauPomeranchuk-Migdal (LPM) effect [ 30, 31]. While the number of scatterings is moderately large, the radiation intensity angular distribution and total energy loss are controlled by the combined effect of the number of scatterings $\bar{n}=L / \lambda$ and the formation probability $p_{f} \sim L / l_{f}=L|\mathbf{k}|^{2} /(2 x E)$ of the gluon in the medium. Here $x$ is the light-cone momentum fraction and $|\mathbf{k}| \ll x E$ is the transverse momentum of the radiated gluon. Therefore, the opacity expansion may converge more rapidly than one would first expect. One of the surprising results derived here and summarized in [28] is that the inclusive induced gluon 
radiation intensity is dominated by the (already quadratic in $L$ ) first order opacity contribution for realistic geometries and jet energies in nuclear collisions.

In GLV1 [ 27], we showed in contrast that in the exclusive tagged case, i.e. with all recoiled partons measured in coincidence with the jet and gluon, a much more complex and nonlinear non-abelian radiation pattern emerges. The tagged recoil partons act as additional color dipole antennas that interfere in a nontrivial way with the jet's color dipole antenna. The resulting color algebra cannot be reduced to simple powers of the color Casimirs. We found that the gluon number distribution for the case of $n_{s}=N$ tagged target partons has the form

$$
d N_{g}^{\left(n_{s}\right)}=d N_{g}^{(0)}\left[1+\left((1+R)^{n_{s}}-1\right) f^{\left(n_{s}\right)}(\kappa, \xi)\right] / Z_{n_{s}},
$$

where $f^{\left(n_{s}\right)}$ depends on two dimensionless ratios $\kappa=\mathbf{k}^{2} / \mu^{2}$ and $\xi=\lambda / l_{f}=\lambda \mu^{2} / 2 x E$, and depends only weakly on $n_{s}$. The color dependence enters through a rapidly increasing function of $R=C_{A} / C_{R}$, the ratio of the Casimirs in the adjoint $\left(d_{A}=N_{c}^{2}-1\right.$ dimensional) gluon representation and the Casimir, $C_{R}$, of the $d_{R}$ dimensional jet representation. In GLV1 unitarity was imposed by a wave function renormalization factor, $Z_{n_{s}}$, that was computed perturbatively. For the inclusive case studied here, unitarity is assured by the inclusion of certain contact double Born (Virtual) terms as emphasized in BDMS [12.

To address energy loss of hard probes produced in nucleus-nucleus collisions we concentrate as in [27, 28 on the case of hard jet produced inside the plasma at a finite point $\left(t_{0}, z_{0}, \mathbf{x}_{0}\right)$ rather than on the GunionBertsch problem [32] in which the jet is replaced by a high energy beam of quarks or gluons prepared in the remote past. We employ as in GW [ 6, 27] static color-screened Yukawa potentials to model interactions in a deconfined quark gluon plasma. The Fourier and color structure of those potentials are assumed to have the form

$$
V_{n}=V\left(q_{n}\right) e^{i q_{n} x_{n}}=2 \pi \delta\left(q^{0}\right) v\left(\overrightarrow{\mathbf{q}}_{n}\right) e^{-i \overrightarrow{\mathbf{q}}_{n} \cdot \overrightarrow{\mathbf{x}}_{n}} T_{a_{n}}(R) \otimes T_{a_{n}}(n),
$$

where $\overrightarrow{\mathbf{x}}_{n}$ is the location of the $n^{\text {th }}$ (heavy) target parton and

$$
v\left(\overrightarrow{\mathbf{q}}_{n}\right) \equiv \frac{4 \pi \alpha_{s}}{\overrightarrow{\mathbf{q}}_{n}^{2}+\mu^{2}}=\frac{4 \pi \alpha_{s}}{\left(q_{n z}+i \mu_{n}\right)\left(q_{n z}-i \mu_{n}\right)},
$$

where $\mu_{n}^{2}=\mu_{n \perp}^{2}=\mu^{2}+\mathbf{q}_{n}^{2}$. The small transverse momentum transfer elastic cross section between the jet and target partons in this model is

$$
\frac{d \sigma_{e l}(R, T)}{d^{2} \mathbf{q}}=\frac{C_{R} C_{2}(T)}{d_{A}} \frac{|v(\mathbf{q})|^{2}}{(2 \pi)^{2}}
$$

In our notation transverse $2 \mathrm{D}$ vectors are denoted as $\mathbf{p}, 3 \mathrm{D}$ vectors as $\overrightarrow{\mathbf{p}}=\left(p_{z}, \mathbf{p}\right)$, and four vectors by $p=\left(p^{0}, \overrightarrow{\mathbf{p}}\right)=\left[p^{0}+p^{z}, p^{0}-p^{z}, \mathbf{p}\right]$.

The color exchange bookkeeping with the target parton $n$ is handled by an appropriate $S U\left(N_{c}\right)$ generator, $T_{a}(n)$, in the $d_{n}$ dimensional representation of the target. $\left(\operatorname{Tr} T_{a}(n)=0\right.$ and $\operatorname{Tr}\left(T_{a}(i) T_{b}(j)\right)=$ $\delta_{i j} \delta_{a b} C_{2}(i) d_{i} / d_{A}$. We will assume that all target partons are in the same $d_{T}$ dimensional representation with Casimir $C_{2}(T)$.) We denote the generators in the $d_{R}$ dimensional representation of the jet by $a \equiv t_{a}$ with $a a=C_{R} 1$. The elastic cross section of the jet with any target parton is therefore proportional to the product of Casimirs, $C_{R} C_{2}(T)$.

The analytic results derived below with the reaction operator approach do not depend on the actual form of $v$, but the Yukawa form will be used for numerical estimates. Recall that in a thermally equilibrated medium at temperature $T$, the color screening mass in pQCD is given by $\mu=4 \pi \alpha_{s} T^{2}$. Also there is a cut-off frequency for soft gluon modes $\omega_{p l} \sim \mu$. We take here $\mu \simeq \omega_{p l} \simeq 0.5 \mathrm{GeV}$ for numerical estimates. In perturbation theory, $\mu^{2} / \lambda \approx 4 \pi \alpha_{s}^{3} \rho$ provides a measure of the $\rho$, the density of plasma partons weighed by appropriate color factors.

This paper is organized as follows: in Sec. II we review the effect of unitarity corrections in the simple elastic jet scattering case. We show how the jet probability is conserved up to first order in opacity through the inclusion of contact double Born graphs. This is the simplest example illustrating how a factor $-\frac{1}{2}$ arises from longitudinal momentum contour integrations in the contact limit. In Sec. III we proceed to the case of gluon bremsstrahlung associated hard probes produced in a dense medium. We discuss the basic diagrammatic rules and specify the assumptions and approximations used in this work. We extend the 
algebraic classification of diagrams given in Ref. [27] to include virtual double Born amplitudes needed in the inclusive case. Sec. III C summarizes the rules of diagrammatic calculus that emerge from detailed analysis of diagrams in Appendix A through Appendix E.

In Sec. IV the new reaction operator formalism is developed. First, operators $\hat{D}_{n}, \hat{V}_{n}$ in Eqs. (76, 81) are constructed from the diagrammatic rules. Products of these operators create partial sums of direct and virtual amplitudes from the initial hard vacuum amplitude. Those partial sums, Eq. (60), form $3^{n}$ classes of diagrams that can be conveniently enumerated via a tensor notation and used to construct recursion relations. In Sec. IV B, the reaction operator, $\hat{R}_{n}=\hat{D}_{n}^{\dagger} \hat{D}_{n}+\hat{V}_{n}+\hat{V}_{n}^{\dagger}$, is constructed to relate the $n^{\text {th }}$ order in opacity inclusive radiation probability distribution to classes of diagrams of order $n-1$. The resulting simple recursion relation, Eq. (97), can be solved in closed form. The general solution, Eq. (101), is suitable for implementation in Monte Carlo event generators to study observable consequences of jet quenching in nuclear collisions.

Color triviality of the inclusive distribution is proven to all orders algebraically with Eq. (101). The proof is much simpler and more transparent than in the path integral formulations [ [13, 20, 34] and is not limited to quark jets.

In Sec IV C a compact general expression for the momentum transfer averaged inclusive distributions, Eq. (113) is derived. Appendix F provides an independent check of this solution through second order starting from the amplitude iteration technique. Numerical results comparing angular distributions of gluons up to the first three orders in opacity are presented in Sec. V A. Analytic and numerical results for the angular integrated intensity distributions are compared in Sec. V B. It is shown that the induced intensity is dominated by the first order in opacity result that is already quadratic in $L$.

A brief summary of these results up to second order in opacity was reported in Ref. [28]. The main result of this paper is the new reaction operator derivation of the solutions, Eq. (101,113), that specify the inclusive non-abelian radiation distribution to any order in opacity.

\section{ELASTIC SCATTERING AND UNITARITY}

To illustrate how the double Born graphs cancel direct contributions to preserve unitarity we review here the simplest case of elastic scattering. Consider a wave packet $j(p)$ of a parton prepared at time $t_{0}$ and localized at $\overrightarrow{\mathbf{x}}_{0}=\left(z_{0}, \mathbf{x}_{0}\right)$ in color representation $R$. The (color matrix) amplitude to measure its momentum as $\overrightarrow{\mathbf{p}}$ in the absence of final state interactions is

$$
M_{0} \equiv i e^{i p x_{0}} j(p) \times \mathbf{1} .
$$

Multiplying $\left|M_{0}\right|^{2}$ by the invariant one particle phase space element $d^{3} \overrightarrow{\mathbf{p}} /\left((2 \pi)^{3} 2|\overrightarrow{\mathbf{p}}|\right)$ and taking the color trace gives the unperturbed inclusive distribution of jets in the wave packet:

$$
d^{3} N_{0}=\operatorname{Tr}\left|M_{0}\right|^{2} \frac{d^{3} \overrightarrow{\mathbf{p}}}{2|\overrightarrow{\mathbf{p}}|(2 \pi)^{3}}=|j(p)|^{2} \frac{d_{R} d^{3} \overrightarrow{\mathbf{p}}}{2|\overrightarrow{\mathbf{p}}|(2 \pi)^{3}} .
$$

Consider next the effect of final state elastic interactions with an array of static potentials localized at $\overrightarrow{\mathbf{x}}_{i}=\left(z_{i}, \mathbf{b}_{i}\right)$ using

$$
H_{I}(t)=\int d^{3} \overrightarrow{\mathbf{x}} \sum_{i=1}^{N} v\left(\overrightarrow{\mathbf{x}}-\overrightarrow{\mathbf{x}}_{1}\right) T_{a}(i) \phi^{\dagger}(\overrightarrow{\mathbf{x}}, t) T_{a}(R) \hat{D}(t) \phi(\overrightarrow{\mathbf{x}}, t)
$$

where $\hat{D}(t)=i \stackrel{\leftrightarrow}{\partial_{t}}$ and $\operatorname{Tr} T_{a}(i) T_{b}(j)=\delta_{i j} \delta_{a b} C_{2}(T) d_{T} / d_{A}$. We will compute the three graphs in Fig. 1. The first order, direct amplitude to scatter with one of the (static) target partons is

$$
M_{1}=i e^{i p x_{0}} \int \frac{d^{4} q}{(2 \pi)^{4}} j(p-q) \Delta(p-q) v(q) D(2 p-q) \sum_{j=1}^{N} e^{i q\left(x_{j}-x_{0}\right)} T_{a}(j) T_{a}(R)
$$

where $\Delta(p) \equiv\left(p^{2}+i \epsilon\right)^{-1}$ and $D(p)=p^{0}$. The sum of double Born amplitudes in the same external potential is 


$$
\begin{array}{r}
M_{2}=i e^{i p x_{0}} T_{a}(R) T_{b}(R) \int \frac{d^{4} q_{1}}{(2 \pi)^{4}} \frac{d^{4} q_{2}}{(2 \pi)^{4}} j\left(p-q_{1}-q_{2}\right) \Delta\left(p-q_{1}-q_{2}\right) D\left(2 p-2 q_{2}-q_{1}\right) v\left(q_{1}\right) \times \\
\times \Delta\left(p-q_{2}\right) D\left(2 p-q_{2}\right) v\left(q_{2}\right) \sum_{j=1}^{N} e^{i\left(q_{1}+q_{2}\right)\left(x_{j}-x_{0}\right)} T_{a}(j) T_{b}(j)
\end{array}
$$
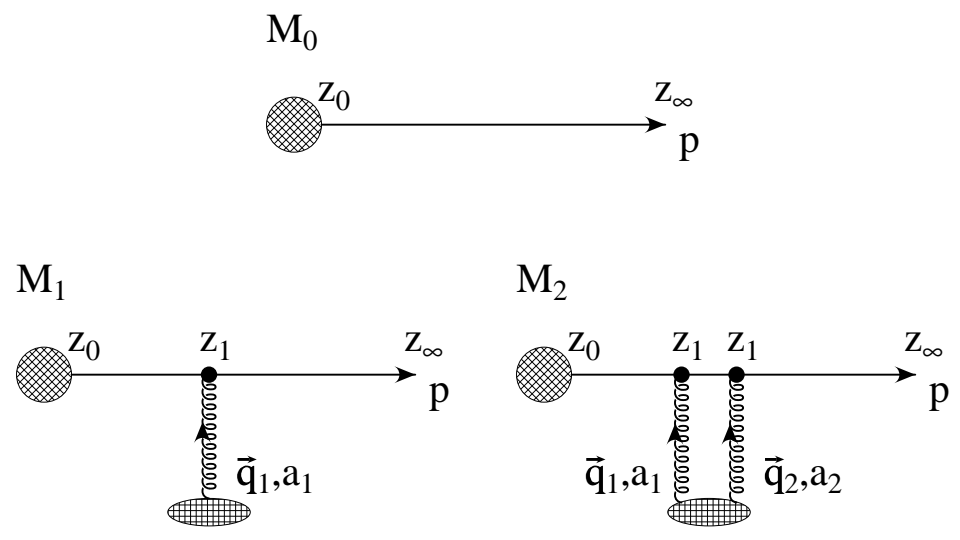

FIG 1. Graphs that produce a jet with $\overrightarrow{\mathbf{p}}$ from an initial wave packet formed at $z_{0}$ followed by single and double Born scattering center located at $z_{1}$.

Double Born with two different centers will not contribute because $\operatorname{Tr} T_{a}(j)=0$. To first order in opacity, the probability distribution of jets is given by

$$
\begin{aligned}
d^{3} N & =\frac{1}{d_{T}} \operatorname{Tr}\left|M_{0}+M_{1}+M_{2}+\cdots\right|^{2} \frac{d^{3} \overrightarrow{\mathbf{p}}}{2|\overrightarrow{\mathbf{p}}|(2 \pi)^{3}} \\
& =d^{3} N_{0}+d^{3} N_{1}+\frac{1}{d_{T}} \operatorname{Tr}\left[2 \operatorname{Re}\left(M_{1} M_{0}^{*}\right)+2 \operatorname{Re}\left(M_{2} M_{0}^{*}\right)\right] \frac{d^{3} \overrightarrow{\mathbf{p}}}{2|\overrightarrow{\mathbf{p}}|(2 \pi)^{3}}+\cdots
\end{aligned}
$$

where we separated the direct $\left(\left|M_{1}\right|^{2}\right)$ and unitary correction contributions with

$$
\begin{aligned}
2|\overrightarrow{\mathbf{p}}|(2 \pi)^{3} \frac{d^{3} N_{1}}{d^{3} \overrightarrow{\mathbf{p}}}= & \int \frac{d^{4} q}{(2 \pi)^{4}} \frac{d^{4} q^{\prime}}{(2 \pi)^{4}} d_{R} j(p-q) j^{*}\left(p-q^{\prime}\right) D(2 p-q) D\left(2 p-q^{\prime}\right) \times \\
& \times \frac{1}{(p-q)^{2}+i \epsilon} \frac{1}{\left(p-q^{\prime}\right)^{2}-i \epsilon} v(q) v^{*}\left(q^{\prime}\right) \frac{C_{R} C_{2}(T)}{d_{A}} \sum_{j=1}^{N} e^{i\left(q-q^{\prime}\right)\left(x_{j}-x_{0}\right)} .
\end{aligned}
$$

To proceed further, we consider a Yukawa potential as in Eqs. (4.5) and assume that all the $x_{j}$ are distributed with the same density

$$
\rho(\overrightarrow{\mathbf{x}})=\frac{N}{A_{\perp}} \bar{\rho}(z)
$$

where $\int d z \bar{\rho}(z)=1$. Second, we assume that the observed $p=(E, E, 0)=\left[E^{+}, 0,0\right]$ is high as compared to the potential screening scale, i.e.

$$
E^{+} \simeq 2 E \gg \mu
$$


We also assume that the distance between the source and scattering centers are large compared to the interaction range:

$$
z_{i}-z_{0} \gg 1 / \mu
$$

Finally, we assume that the source current or packet $j(p)$ varies slowly over the range of momentum transfers supplied by the potential. We can then approximate the $q_{z}$ contour integrals ignoring the $q_{z}$ dependence of the source $j(p \pm q)$. With $(16)$ we can also neglect the contributions to the $q_{z}$ contour integrals due to singularities of the potentials at $\pm i \mu_{\perp}, \mu_{\perp}^{2}=\mu^{2}+\mathbf{q}^{2}$. With these simplifying assumptions, only the residues with $q_{z} \approx-i \epsilon+\mathbf{q}^{2} / E^{+}$and $q_{z}^{\prime} \approx+i \epsilon+\mathbf{q}^{\prime 2} / E^{+}$contribute:

$$
\begin{aligned}
2|\overrightarrow{\mathbf{p}}|(2 \pi)^{3} \frac{d^{3} N_{1}}{d^{3} \overrightarrow{\mathbf{p}}}= & N d_{R} \int \frac{d q_{z} d^{2} \mathbf{q}}{(2 \pi)^{3}} \frac{d q_{z}^{\prime} d^{2} \mathbf{q}^{\prime}}{(2 \pi)^{3}} j(p-q) j^{*}\left(p-q^{\prime}\right) v(\mathbf{q}) v^{*}\left(\mathbf{q}^{\prime}\right) \frac{C_{R} C_{2}(T)}{d_{A}} \times \\
& \times \frac{E^{+}}{E^{+} q_{z}-q_{z}^{2}-\mathbf{q}^{2}+i \epsilon} \frac{E^{+}}{E^{+} q_{z}^{\prime}-q_{z}^{\prime 2}-\mathbf{q}^{\prime 2}-i \epsilon}\left\langle e^{-i\left(\overrightarrow{\mathbf{q}}-\overrightarrow{\mathbf{q}}^{\prime}\right) \cdot\left(\overrightarrow{\mathbf{x}}_{1}-\overrightarrow{\mathbf{x}}_{0}\right)}\right\rangle \\
\approx & N d_{R} \int \frac{d^{2} \mathbf{q}}{(2 \pi)^{2}} \frac{d^{2} \mathbf{q}^{\prime}}{(2 \pi)^{2}} j(p-\mathbf{q}) j^{*}\left(p-\mathbf{q}^{\prime}\right)\left\langle e^{-i\left(\mathbf{q}-\mathbf{q}^{\prime}\right) \cdot\left(\mathbf{x}_{1}-\mathbf{x}_{0}\right)}\right\rangle v(\mathbf{q}) v^{*}\left(\mathbf{q}^{\prime}\right) \frac{C_{R} C_{2}(T)}{d_{A}}
\end{aligned}
$$

A major simplification (that we will also use in the radiation case) occurs if the relative transverse coordinate (impact parameter) $\mathbf{b}=\mathbf{x}_{i}-\mathbf{x}_{0}$ varies over a large transverse area, $A_{\perp}$, relative to the interaction area $1 / \mu^{2}$. In this case, the ensemble average over the scattering center location reduces to an impact parameter average as follows:

$$
\langle\cdots\rangle=\int \frac{d^{2} \mathbf{b}}{A_{\perp}} \cdots
$$

The ensemble average over the phase factor in Eq. (17) then yields

$$
\left\langle e^{-i\left(\mathbf{q}-\mathbf{q}^{\prime}\right) \cdot \mathbf{b}}\right\rangle=\frac{(2 \pi)^{2}}{A_{\perp}} \delta^{2}\left(\mathbf{q}-\mathbf{q}^{\prime}\right)
$$

Note that we ignored a small phase shift in Eq. (17) $\propto \exp \left[-\mathbf{q}^{2}\left(z_{1}-z_{0}\right) / E^{+}\right] \approx 1$ on account of our high energy (eikonal) assumption. The ensemble averaged first order in opacity direct contribution to the jet distribution therefore reduces to the familiar form noting Eq. (6)

$$
\begin{aligned}
\frac{2|\overrightarrow{\mathbf{p}}|(2 \pi)^{3}}{d_{R}} \frac{d^{3} N_{1}}{d^{3} \overrightarrow{\mathbf{p}}} & \approx \frac{N}{A_{\perp}} \int \frac{d^{2} \mathbf{q}}{(2 \pi)^{2}}|j(p-\mathbf{q})|^{2} \frac{d \sigma_{e l}(R, T)}{d^{2} \mathbf{q}} \\
& \approx|j(p)|^{2} \int d^{2} \mathbf{q} \frac{N}{A_{\perp}} \frac{d \sigma_{e l}(R, T)}{d^{2} \mathbf{q}}=\frac{L}{\lambda}|j(p)|^{2}
\end{aligned}
$$

where the last line only holds if the initial packet is very wide in momentum space compared to the momentum transfer scale $\mu$.

Next we turn next to the unitary corrections. The first order term $\left\langle M_{1} M_{0}^{*}\right\rangle=0$ on account of $\operatorname{Tr} T_{a}(j)=0$. A non-vanishing unitarity correction arises however from

$$
\begin{aligned}
\frac{1}{d_{T}} \operatorname{Tr}\left\langle M_{2} M_{0}^{*}\right\rangle= & \int \frac{d^{4} q_{1}}{(2 \pi)^{4}} \frac{d^{4} q_{2}}{(2 \pi)^{4}} j\left(p-q_{1}-q_{2}\right) j^{*}(p) D\left(2 p-q_{2}\right) D\left(2 p-q_{1}-q_{2}\right) \times \\
& \times \frac{V\left(q_{2}\right)}{\left(p-q_{1}-q_{2}\right)^{2}+i \epsilon} \frac{V\left(q_{1}\right)}{\left(p-q_{2}\right)^{2}+i \epsilon} \operatorname{Tr}\left(T_{a}(R) T_{b}(R)\right) \\
& \times\left\langle\sum_{j=1}^{N} e^{i\left(q_{1}+q_{2}\right)\left(x_{j}-x_{0}\right)} \frac{1}{d_{T}} \operatorname{Tr}\left(T_{a}(j) T_{b}(j)\right)\right\rangle .
\end{aligned}
$$

Note that the impact parameter average constrains $\mathbf{q}_{1}+\mathbf{q}_{2}=0$ in this case $\left(q_{1}^{0}=q_{2}^{0}=0\right.$ for static potentials). The phase factor requires that we close the $q_{1 z}$ contour in the lower half-plane. We pick up 
the $-2 \pi i \operatorname{Res}\left(q_{1 z}=-i \epsilon-q_{2 z}\right)$. This results in setting $\overrightarrow{\mathbf{q}}_{1}+\overrightarrow{\mathbf{q}}_{2}=0$ throughout. Again the residue from the second pole in the lower half-plane $q_{z}=-i \mu_{\perp}$ is suppressed by the phase factor. We are left with

$$
\frac{1}{d_{T}} \operatorname{Tr}\left\langle M_{2} M_{0}^{*}\right\rangle \approx-i N d_{R}|j(p)|^{2} \int \frac{d^{2} \mathbf{q}}{(2 \pi)^{2}} \frac{d q_{2 z}}{(2 \pi)} \frac{\left(4 \pi \alpha_{s}\right)^{2}}{A_{\perp}} \frac{C_{R} C_{2}(T)}{d_{A}} \frac{1}{\left(q_{2 z}^{2}+\mu_{\perp}^{2}\right)^{2}} \frac{E^{+}}{E^{+} q_{2 z}-q_{2 z}^{2}-\mathbf{q}^{2}+i \epsilon}
$$

where $\mu_{\perp}^{2}=\mu^{2}+\mathbf{q}^{2}$. The $q_{2 z}$ can be performed yielding

$$
\int \frac{d q_{2 z}}{2 \pi} \frac{1}{\left(q_{2 z}^{2}+\mu_{\perp}^{2}\right)^{2}} \frac{E^{+}}{E^{+} q_{2 z}-q_{2 z}^{2}-\mathbf{q}^{2}+i \epsilon} \approx \frac{-i}{2} \frac{1}{\left(\mathbf{q}^{2}+\mu^{2}\right)^{2}} .
$$

The double Born "contact" contribution to the differential yield at first order in opacity is therefore

$$
\frac{1}{d_{T}} \operatorname{Tr} 2 \operatorname{Re}\left\langle M_{2} M_{0}^{*}\right\rangle \approx-\frac{L}{\lambda}|j(p)|^{2} d_{R} .
$$

The inclusive first order in opacity elastic distribution is therefore

$$
\begin{aligned}
\frac{2|\overrightarrow{\mathbf{p}}|(2 \pi)^{3}}{d_{R}} \frac{d^{3} N}{d^{3} \overrightarrow{\mathbf{p}}} & =|j(p)|^{2}\left(1-\frac{L}{\lambda}\right)+\frac{N}{A_{\perp}} \int \frac{d^{2} \mathbf{q}}{(2 \pi)^{2}}|j(p-\mathbf{q})|^{2} \frac{d \sigma_{e l}(R, T)}{d^{2} \mathbf{q}}+O\left(\frac{L}{\lambda}\right)^{2} \\
& =|j(p)|^{2}+\int d z \rho(z, 0) \int \frac{d^{2} \mathbf{q}}{(2 \pi)^{2}}\left\{\frac{d \sigma_{e l}(R, T)}{d^{2} \mathbf{q}}-\sigma_{e l} \delta^{2}(\mathbf{q})\right\}|j(p-\mathbf{q})|^{2}+O\left(\frac{L}{\lambda}\right)^{2} .
\end{aligned}
$$

This is the first order term in the Glauber multiple scattering series. Note that when integrated over momentum, the double Born term cancels exactly direct contribution and therefore enforces probability conservation. However, for very narrow coordinate space packets, i.e. wide in momentum space so that $|j(p-\mathbf{q})|^{2} \approx|j(p)|^{2}$, we see that the double Born contribution actually cancels approximately the first order direct term differentially.

We derived this cancelation assuming $\mathbf{p}=0$ throughout, but it holds generally since all we needed was that the phase shift $\left(z_{i}-z_{0}\right)(\mathbf{q}-\mathbf{p})^{2} / E^{+} \ll 1$. The point of this brief review was to emphasize that the unitarity cancelation arose due to the factor of $\frac{1}{2}$ that appeared in Eq. (23) from the longitudinal momentum contour integration and from the factor -1 that appeared due to moving the potential from one side of a cut to another. This is a generic property of contact interactions that also holds in the radiation case also as pointed out in [12] and derived here in the Appendix.

\section{SOFT GLUON RADIATION}

\section{A. Kinematics, triple gluon vertices, and color algebra}

Consider a source $J$ that produces a jet that subsequently radiate a gluon with four momentum $k$, polarization $\epsilon(k)$, and emerges with momentum $p$. In light-cone components

$$
k=\left[x E^{+}, k^{-} \equiv \omega_{0}, \mathbf{k}\right], \epsilon(k)=\left[0,2 \frac{\boldsymbol{\epsilon} \cdot \mathbf{k}}{x E^{+}}, \boldsymbol{\epsilon}\right], \quad p=\left[(1-x) E^{+}, p^{-}, \mathbf{p}\right] .
$$

Soft radiation is defined as $x \ll 1$ so that, for example, $p^{+} \gg k^{+}$and $p^{-}=\mathbf{p}^{2} /(1-x) E^{+} \ll k^{-}=\omega_{0}=$ $\mathbf{k}^{2} / x E^{+}$.

We adopt the shorthand notation of Refs. [26, 27]

$$
\omega_{0}=\frac{\mathbf{k}^{2}}{2 \omega}, \omega_{i}=\frac{\left(\mathbf{k}-\mathbf{q}_{i}\right)^{2}}{2 \omega}, \omega_{(i j)}=\frac{\left(\mathbf{k}-\mathbf{q}_{i}-\mathbf{q}_{j}\right)^{2}}{2 \omega}, \omega_{(i \cdots j)}=\frac{\left(\mathbf{k}-\sum_{m=i}^{j} \mathbf{q}_{m}\right)^{2}}{2 \omega} .
$$

In the soft eikonal kinematics, that we consider

$$
E^{+} \gg k^{+} \gg \omega_{(i \cdots j)} \gg \frac{(\mathbf{p}+\mathbf{k})^{2}}{E^{+}} .
$$


The soft gluon and soft rescattering approximations will allow us to simplify analytic results using:

$$
\begin{aligned}
J(p-Q+k) & \approx J(p+k) \approx J(p), \\
\epsilon_{\mu}(k)(p-Q+k)^{\mu} & \approx \frac{\boldsymbol{\epsilon} \cdot \mathbf{k}}{x}, \\
k(p-Q) & \approx k p \approx \frac{\mathbf{k}^{2}}{2 x}, \\
\Delta(p-Q) \Delta(p-Q+k) & \approx \frac{1}{k p}(\Delta(p-Q)-\Delta(p-Q+k)) .
\end{aligned}
$$

Scattering of the jet or radiated gluon with the potential centered at position $\overrightarrow{\mathbf{x}}_{n}$ introduces an integration $\int d^{4} q_{n} /(2 \pi)^{4}$ in the amplitude over the potential $V\left(q_{n}\right)$. We use the GW model potential Eqs. (4), 5 ). If the scattering involves a momentum exchange with the high energy jet, the vertex factor is simply $-i E^{+}$in the eikonal limit. Neglecting the spin of the jet parton, each intermediate jet propagator brings in a factor $+i \Delta(p-Q)$, where $\Delta(p)=1 /\left(p^{2}+i \epsilon\right)$. The gluon emission vertex then gives in this approximation a factor $+i g_{s}(2 p+k-2 Q)^{\mu}$, where $Q$ is the sum of the subsequent momentum transfers.

For scattering of the radiated gluon, consider the triple gluon tensor, $\Gamma^{\alpha 0 \gamma}(k, q)$ for a $g(k-q, \alpha, a)+V(q, \beta=$ $0, b) \rightarrow g(k, \gamma, c)$. In our model, $q_{n}^{0}=0,\left|q_{n z}\right|=\left|q_{n}^{+}\right|=\left|q_{n}^{-}\right| \ll\left|\mathbf{q}_{n}\right|$, and thus the $q_{n}$ correspond to small (almost) transverse momentum transfers. If the last interaction of the gluon is with center $m$, then that last vertex is

$$
\Gamma^{\alpha 0 \gamma}\left(k, q_{m}, k-q_{m}\right)=2 \omega g^{\alpha \gamma}-\left(k+q_{m}\right)^{\alpha} g^{0 \gamma}+\left(2 q_{m}-k\right)^{\gamma} g^{0 \alpha} .
$$

The color factor for the above "clockwise" kinematic convention is $-f^{c b a}$.

The contraction of Eq. (30) with the final polarization gives

$$
\Gamma^{\alpha}\left(k ; q_{m}\right) \equiv \Gamma^{\alpha \gamma}\left(k, q_{m}\right) \epsilon_{\gamma}(k)=2 \omega \epsilon^{\alpha}-\left(k+q_{m}\right)^{\alpha} \epsilon^{0}+2 g^{0 \alpha}\left(q_{m} \epsilon\right) .
$$

For the case of two gluon rescatterings the two vertices combine

$$
\begin{aligned}
\Gamma^{\alpha}\left(k ; q_{n}, q_{m}\right) \equiv & \Gamma^{\alpha \mu}\left(k-q_{m}, q_{n}\right) g_{\mu \nu} \Gamma^{\nu}\left(k ; q_{m}\right) \\
\Gamma^{\alpha}\left(k ; q_{n}, q_{m}\right)= & 4 \omega^{2} \epsilon^{\alpha}-\omega\left(3 k+q_{n}+q_{m}\right)^{\alpha} \epsilon^{0}+g^{0 \alpha}\left(4 \omega\left(q_{n}+q_{m}\right) \epsilon\right) \\
& -g^{0 \alpha}\left(2 q_{n}\left(k-q_{m}\right)+q_{m}^{2}\right) \epsilon^{0}-2\left(k-q_{m}+q_{n}\right)^{\alpha}\left(q_{m} \epsilon\right) .
\end{aligned}
$$

Note that using $+g^{\mu \nu}$ to combine the vertices above requires us to insert $-i \Delta(k-Q)$ for each gluon propagator, where use $\Delta(p)=1 /\left(p^{2}+i \epsilon\right)$.

The gluon vertex factors contracted by emission vertex factors then give

$$
\begin{aligned}
\Gamma_{m} & \equiv\left(2 p+k-q_{m}\right)_{\alpha} \Gamma^{\alpha}\left(k ; q_{m}\right) \approx 2 E^{+}\left(\boldsymbol{\epsilon} \cdot\left(\mathbf{k}-\mathbf{q}_{m}\right)\right), \\
\Gamma_{m n} & \equiv\left(2 p+k-q_{m}-q_{n}\right)_{\alpha} \Gamma^{\alpha}\left(k ; q_{n}, q_{m}\right) \approx 2 E^{+} k^{+}\left(\boldsymbol{\epsilon} \cdot\left(\mathbf{k}-\mathbf{q}_{m}-\mathbf{q}_{n}\right)\right) .
\end{aligned}
$$

We neglected above corrections of $\mathcal{O}(x)$. Eq.(33) also applies approximately if the emission occurs while the jet is off shell with $p-Q$, with $Q^{\mu}=(0, \delta \mathbf{q})$ total momentum transfer still to be acquired from subsequent jet re-interactions. Replacing $p$ by $p-Q$ adds for example $2\left(k^{+}\right)^{2} \boldsymbol{\epsilon} \cdot \mathbf{q}$ which is again $\mathcal{O}\left(x=k^{+} / E^{+}\right)$smaller than the terms retained above.

The full triple glue vertices including coupling and color algebra for producing a final color $c$ and initial color $a$ followed by color potential interactions $a_{m}$ and $a_{m}$ are then given by

$$
\begin{aligned}
\Lambda_{m} & \equiv \Gamma_{m}\left(-f^{c a_{m} a}\right)\left(i g_{s} t_{a}\right)\left(T_{a_{m}}(m)\right) \\
& \approx-2 g_{s} E^{+} \boldsymbol{\epsilon} \cdot\left(\mathbf{k}-\mathbf{q}_{m}\right)\left[c, a_{m}\right] T_{a_{m}}(m) \\
\Lambda_{m n} & \equiv \Gamma_{m n}\left(-f^{c a_{n} e}\right)\left(-f^{e a_{m} a}\right)\left(i g_{s} t_{a}\right)\left(T_{a_{n}}(n)\right)\left(T_{a_{m}}(m)\right) \\
& \approx-2 i g_{s} E^{+} k^{+} \boldsymbol{\epsilon} \cdot\left(\mathbf{k}-\mathbf{q}_{1}-\mathbf{q}_{2}\right)\left[\left[c, a_{n}\right], a_{m}\right]\left(T_{a_{n}}(n) T_{a_{m}}(m)\right) .
\end{aligned}
$$

To complete the Feynman rules for our problem, we note that there is a factor

$$
i J(p+k-Q) e^{i(p+k-Q) x_{0}}
$$


for the jet production vertex at $x_{0}^{\mu}$ if the net subsequent target momentum transfer to the jet plus gluon system is $Q$. The hard jet radiation amplitude to emit a gluon with momentum, polarization, and color $(k, \epsilon, c)$ without final state interactions is then

$$
\begin{aligned}
M_{0} & =i J(p+k) e^{i(p+k) x_{0}}\left(i g_{s}\right)(2 p+k)_{\mu} \epsilon^{\mu}(k) i \Delta(p+k) c \\
& \approx J(p+k) e^{i(p+k) x_{0}}\left(-2 i g_{s}\right) \frac{\boldsymbol{\epsilon} \cdot \mathbf{k}}{k^{2}} c \approx J(p) e^{i p x_{0}}\left(-2 i g_{s}\right) \frac{\boldsymbol{\epsilon} \cdot \mathbf{k}}{k^{2}} e^{i \omega_{0} z_{0}} c,
\end{aligned}
$$

corresponding to Eqs. $(9,10)$ in Ref. [27].

\section{B. Graphical shorthand}

In Ref. [26] we introduced the following notation, $M_{n_{s}, m, l}$ for the direct (tagged) $n_{s}$ scattering center amplitude for emitting a gluon between points $z_{m}$ and $z_{m+1}$ with a final state interaction pattern encoded by $l=\sum_{1}^{n_{s}} \sigma_{i} 2^{i-m-1}$, with $\sigma_{i}=0,1$ depending on whether the interaction was with the jet of the gluon. To include virtual double Born corrections, it convenient to associate a more specific graphical coding to keep track of the many different possibilities..

A graph consists of a gluon emission vertex, $G_{m}$, for emitting a gluon between centers $z_{m}$ and $z_{m+1}$ as before, but also a specific set of direct interactions, $X_{i, \sigma_{i}}$ with $\sigma_{i}=0,1$ for center $i$, and double Born interactions denoted, $O_{j, a_{j}}$, for center $j$. The index $a_{j}=0,1,2$ denotes a contact interaction at center $j$ with the jet, gluon, and both jet+gluon respectively. In this notation

$$
M_{n, m, l}=\left[\prod_{i=0}^{m} X_{i, 0}\right] G_{m}\left[\prod_{j=m+1}^{n} X_{j, \sigma_{j}}\right]
$$

here $\sigma_{j}=0,1$ is the $\mathrm{j}^{\text {th }}$ binary bit in the expansion of $l \times 2^{m+1}$. For example, the graph corresponding to gluon emission before the first scattering center followed by a jet interaction with center " 1 " and the gluon rescattering at center " 2 " is given by $M_{2,0,2}=G_{0} X_{1,0} X_{2,1}$. This is simply an algebraic way of representing the corresponding graph. Similarly, $M_{0,0,0}=G_{0}$ and $M_{1,0,1}=G_{0} X_{1,1}$. This notation is particularly useful to specify more complicated diagrams that arise from multiple direct and double Born interactions. It extends the algebraic approach to the topologically distinct graphs of Ref. [27] by the inclusion of virtual corrections. Any diagram can be written in the form

$$
M=\left[\prod_{i=0}^{m} T_{i, \alpha_{i}}\right] G_{m}\left[\prod_{i=m+1}^{n} T_{j, \beta_{j}}\right], \quad T_{i, \alpha_{i}}, T_{i, \beta_{i}} \in\left(X_{i, \sigma_{i}}, O_{i, a_{i}}\right)
$$

While Eq. (37) can be used to enumerate all "single gluon emission with rescatterings" diagrams arising from a target with $n$ aligned centers, it will prove convenient to group diagrams into classes of graphs that can be iteratively built from a diagrammatic kernel. The two important kernels correspond to the two distinct possibilities for preparing an off-shell parton. In the first case the jet originates asymptotically at infinity and then enters the medium. It needs at least one inelastic interaction to get off-shell. This is the more extensively studied Gunion-Bertsch (GB) limit [10, 11, 32]. Depending on whether the first interaction in the plasma is real or virtual

$$
\operatorname{Ker}^{(G B)}= \begin{cases}B_{1}^{(R)}=-2 i g_{s} \boldsymbol{\epsilon} \cdot\left(\frac{\mathbf{k}}{\mathbf{k}^{2}}-\frac{\mathbf{k}-\mathbf{q}_{1}}{\left(\mathbf{k}-\mathbf{q}_{1}\right)^{2}}\right) e^{i \omega_{0} z_{1}}\left[c, a_{1}\right], & \text { Real initiator } \\ B_{1}^{(V)}=-2 i g_{s} \frac{C_{A}}{2} \boldsymbol{\epsilon} \cdot\left(\frac{\mathbf{k}}{\mathbf{k}^{2}}-\frac{\mathbf{k}-\mathbf{q}_{1}}{\left(\mathbf{k}-\mathbf{q}_{1}\right)^{2}}\right) e^{i \omega_{0} z_{1}} c, & \text { Virtual initiator }\end{cases}
$$

is the effective color current [6, 10] (here given in the eikonal approximation in the small $x$ limit). In Eq. (38) $c$ and $a_{1}$ are the color matrices of the radiated gluon and the soft momentum transfer.

In the second and more experimentally relevant case, the parton is produced inside the medium (e.g., through $A+A \rightarrow q+\bar{q}+X$ with high $Q^{2} \equiv E^{+2}$ ) with accompanying gluon radiation. The kernel or initiator for the hard production vertex is 


$$
\operatorname{Ker}^{(H)}=G_{0}=-2 i g_{s} \frac{\boldsymbol{\epsilon} \cdot \mathbf{k}}{\mathbf{k}^{2}} e^{i \omega_{0} z_{0}} c,
$$

where $c$ the color (generator) of the radiated the gluon. Classes for both Hard and Gunion-Bertsch cases can be constructed starting from the appropriate kernel. We focus here the hard jet case relevant to nuclear collisions with the hard production vertex localized at $z_{0}$, as in Eq. (39). We consider the effect of final state interactions at positions $z_{i}>z_{0}$ along the direction of the jet.

Let $\mathcal{A}$ denote a class of graphs with $N_{\mathcal{A}}$ members in which the last interaction has occured at position $z_{j}<z_{i}$. Each class includes one special graph, denoted $\mathcal{A}_{0}$, where the gluon is emitted after all real and virtual interactions in that class. We can enlarge this class of graphs to include a direct hit at $z_{i}$ and label it by $\mathcal{A} D_{i}$, where $D_{i}$ specifies the direct insertion iteration. This new class contains $2 N_{\mathcal{A}} \operatorname{diagrams} \mathcal{A} X_{i, 0}$ and $\mathcal{A} X_{i, 1}$ obtained by a direct interaction of either the jet or the previously emitted gluon. In addition, there is new special diagram, $\mathcal{A} G^{-1} X_{i, 0} G_{i}=\left(\mathcal{A} D_{i}\right)_{0}$, where all interactions (direct or virtual) including the one at $z_{i}$ are with the jet and the gluon is emitted after all interactions at $z_{i}<z<\infty$. $\left(G^{-1}\right.$ amputates the gluon emission vertex of $\mathcal{A}_{0}$.) The new class with $2 N_{\mathcal{A}}+1$ graphs is constructed operationally as

$$
\mathcal{A} \Rightarrow \mathcal{A} D_{i}=\mathcal{A} X_{i, 0}+\mathcal{A} X_{i, 1}+\mathcal{A} G^{-1} X_{i, 0} G_{i}
$$

Starting with $\mathcal{A}=G_{0}, \mathcal{A} D_{1} \cdots D_{n_{s}}$ is then the set of $2^{n_{s}+1}-1$ direct interactions with centers " 1 " through " $n_{s} "$ are constructed in [27].

Similarly we can consider the possibilities that arise from inserting a double Born hit at location $z_{i}$. This case differs from the above only in that now a new subclass appears because one of the legs can be attached to the jet line and the other one to the gluon line, i.e. $\mathcal{A} O_{i, 2}$. The new class $\mathcal{A} V_{i}$ with $3 N_{\mathcal{A}}+1$ graphs including these virtual interactions at center $i$ are constructed operationally as

$$
\mathcal{A} \Rightarrow \mathcal{A} V_{i}=\mathcal{A} O_{i, 0}+\mathcal{A} O_{i, 1}+\mathcal{A} O_{i, 2}+\mathcal{A} G^{-1} O_{i, 0} G_{i}
$$

The classes and conjugate classes contributing to the gluon radiation distribution in the opacity expansion at order $(L / \lambda)^{n} \propto\left(\sigma_{e l} / A_{\perp}\right)^{n}$ for $n=0,1,2$ are listed in the table below. Each class is obtained through ordered insertions from the hard kernel $\operatorname{Ker}^{(H)}$ indicated by $G_{0}$.

1. $(L / \lambda)^{0} \propto\left(\sigma_{e l} / A_{\perp}\right)^{0}$ contributions

- $1\left(n_{s}=0\right) \times\left(n_{s}=0\right)^{\dagger}$

$$
G_{0} G_{0}^{\dagger} 1 \text { graph }
$$

2. $(L / \lambda)^{1} \propto\left(\sigma_{e l} / A_{\perp}\right)^{1}$ contributions

- $4\left(n_{s}=2\right) \times\left(n_{s}=0\right)^{\dagger}$

$$
G_{0} V_{1} G_{0}^{\dagger} \quad 4 \text { graphs }
$$

- $9\left(n_{s}=1\right) \times\left(n_{s}=1\right)^{\dagger}$

$$
G_{0} D_{1} D_{1}^{\dagger} G_{0}^{\dagger} 9 \text { graphs }
$$

3. $(L / \lambda)^{2} \propto\left(\sigma_{e l} / A_{\perp}\right)^{2}$ contributions

- $13\left(n_{s}=4\right) \times\left(n_{s}=0\right)^{\dagger}$

$$
G_{0} V_{1} V_{2} G_{0}^{\dagger} 13 \text { graphs }
$$

- $57\left(n_{s}=3\right) \times\left(n_{s}=1\right)^{\dagger}$

$$
\begin{array}{ll}
G_{0} D_{1} V_{2} D_{1}^{\dagger} G_{0}^{\dagger} & 30 \text { graphs } \\
G_{0} V_{1} D_{2} D_{2}^{\dagger} G_{0}^{\dagger} & 27 \text { graphs }
\end{array}
$$


- $65\left(n_{s}=2\right) \times\left(n_{s}=2\right)^{\dagger}$

$$
\begin{array}{rr}
G_{0} D_{1} D_{2} D_{2}^{\dagger} D_{1}^{\dagger} G_{0}^{\dagger} & 49 \text { graphs } \\
G_{0} V_{1} V_{2}^{\dagger} G_{0}^{\dagger} & 16 \text { graphs }
\end{array}
$$

The left column sorts contributions in terms of diagrams with fixed number of interactions $n_{s}, n_{s}^{\prime}$ in the amplitude and complex conjugate amplitude. For example, there are 57 contributions involving amplitudes with 3 interactions times complex conjugate aplitudes with 1 interaction that contribute to second order in opacity. Since two of the three interactions in the amplitude must involve the same center, these 57 diagrams subdivide naturally into the two class structures (46,47) indicated on the right column. We note that (42), (44) and (48) correspond to the direct diagrams computed in [27] for the exclusive tagged spectrum.

\section{Diagrammatic calculus}

Whereas the time-ordered perturbation techniques [ 10] used in [27] greatly facilitated the listing of higher order contributions, it is useful to show how those shortcuts emerged from Feynman diagrams especially to check the shortcut rules that apply to the double Born contributions. Detailed diagramatic evaluation from which those rules emerge are presented in Appendix A through Appendix E. They exhaust all possibilities that we can encounter for single and double Born interactions in a potential model with multiple centers. It is important to note that a double Born interaction at position $z_{i}$ can be thought of as the "contact" limit of two direct hits at positions $z_{i}$ and $z_{i+1}$ respectively

$$
\mathcal{A} O_{i, a_{i}} \propto \lim _{z_{i+1} \rightarrow z_{i}} \mathcal{A} X_{i, b_{i}} X_{i+1, b_{i+1}} .
$$

We summarize here the kinematical and color parts of this diagrammatic calculus:

- In all diagrams the jet production factors out in the form

$$
J(p) e^{i p x_{0}}
$$

under the soft-gluon soft-interaction assumption Eq. (29). Therefore the jet distribution can be factored out under the assumption that $J$ is broad.

- For graphs involving direct interactions only, i.e. of class $G_{0} D_{1} D_{2} \cdots D_{i} \cdots$, the Feynman diagrams reproduce the time ordered perturbation results listed in Ref. [27]. The simplest one interaction example is discussed in Appendix A. We note that although Appendix B through Appendix E are aimed at clarifying the contact limits, their intermediate results in the well-separated $z_{i+1}-z_{i}=\lambda \gg 1 / \mu$ case are directly comparable to [27.

- A diagram which involves a contact contribution of the form $\mathcal{A} O_{i, 0}$ or $\mathcal{A} O_{i, 1}$, i.e. both interactions are on a single line, is kinematically identical to a diagram in the well-separated case, $\propto X_{i, 0} X_{i+1,0}$ and $\propto X_{i, 1} X_{i+1,1}$ respectively, but with $z_{i}=z_{i+1}$ (50). This can be seen in from Appendix B for the final state virtual interaction of the radiated gluon and in Appendix $\mathrm{C}$ for a virtual final state scattering of the jet. There is, however, a multiplicative factor of $\frac{1}{2}$ per contact interaction that emerges from contour integrals. Eq. (B8) shows how the strength interpolates between 1 and $\frac{1}{2}$ as the separation varies for a Yukawa potential. Eq. (B15) shows the generality of the factor $\frac{1}{2}$ in the contact limit.

- For the case when one of the legs in the contact contribution is attached to the jet line and the other to the gluon line, i.e. $\mathcal{A} O_{i, 2}$, the amplitude is kinematically identical to a diagram $\mathcal{A} X_{i, 0} X_{i+1,1}$ in the well-separated case with $z_{i}=z_{i+1}$ (50). There are no additional numerical factors for such mixed contact term as shown in Appendix D. We also note that the contact limit of the diagram $\propto X_{i, 1} X_{i+1,0}$ should not be added to avoid over counting (i.e, they are topologically identical in the contact limit).

- We show in Appendix E that the class of diagrams that have the gluon emission vertex between the two momentum transfers of a double Born interaction are suppressed in the "contact" limit by factors $\mathcal{O}\left(k^{+} / E^{+}\right)$and $\mathcal{O}\left(\mu / k^{+}\right)$and thus can be neglected in the framework of our approximations (28). This verifies the naive argument from time-ordered perturbation theory that such diagrams are of zero-measure and vanish in the contact limit. 
- Each power of the potential comes with a weight (elastic scattering amplitude)

$$
(-i) \int \frac{d^{2} \mathbf{q}_{i}}{(2 \pi)^{2}} v\left(0, \mathbf{q}_{i}\right) e^{-i \mathbf{q}_{i} \cdot \mathbf{b}_{i}} T_{a_{i}}(i),
$$

that factors out as well under the assumptions $(29,28)$ and is still to be integrated over. The amplitudes above eventually build into the elastic scattering cross section $\propto \sigma_{e l}$.

- The appropriate color factor for the jet+gluon part can be constructed following Ref. [27]. In the case of a virtual interaction certain simplifications, namely

$$
a_{i} a_{i}=C_{R} \mathbf{1}, \quad a_{i}\left[c, a_{i}\right]=-\frac{C_{A}}{2} c
$$

can occur even at the amplitude level.

- The last critical rule that emerges is that direct terms come with a plus sign while each virtual interaction contributes a minus.

These rules are illustrated to compute the classes of amplitudes needed up to second order in opacity in Appendix F.

\section{Impact parameter averages}

Consider next the averaging over the transverse impact parameters $\mathbf{b}_{i}=\mathbf{x}_{i}-\mathbf{x}_{0}$. A typical term that appears to second order in opacity is $G_{0} X_{1,0} O_{2,2} X_{1,0}^{\dagger} G_{0}^{\dagger}$. This involves one direct interaction with center " 1 " and a double Born with center " 2 ". We consider separately the average over $\mathbf{b}_{i}$ involving direct and virtual interaction centers.

For a scattering center, $i$, that appears in a direct interaction the impact parameter average takes the form

$$
\begin{aligned}
\langle\cdots\rangle_{A_{\perp}} & =\left\langle\cdots \int \frac{d^{2} \mathbf{b}_{i}}{A_{\perp}}(-i) \int \frac{d^{2} \mathbf{q}_{i}}{(2 \pi)^{2}} v\left(0, \mathbf{q}_{i}\right) e^{-i \mathbf{q}_{i} \cdot \mathbf{b}_{i}}(+i) \int \frac{d^{2} \mathbf{q}_{i}^{\prime}}{(2 \pi)^{2}} v^{*}\left(0, \mathbf{q}_{i}^{\prime}\right) e^{+i \mathbf{q}_{i}^{\prime} \cdot \mathbf{b}_{i}} \cdots\right\rangle \\
& =\cdots \int \frac{d^{2} \mathbf{q}_{i}}{(2 \pi)^{2}} \frac{d^{2} \mathbf{q}_{i}^{\prime}}{(2 \pi)^{2}} \frac{(2 \pi)^{2} \delta^{2}\left(\mathbf{q}_{i}-\mathbf{q}_{i}^{\prime}\right)}{A_{\perp}} v\left(0, \mathbf{q}_{i}\right) v\left(0, \mathbf{q}_{i}^{\prime}\right) \cdots \\
& =\cdots \frac{\sigma}{A_{\perp}} \int \frac{d^{2} \mathbf{q}_{i}}{(2 \pi)^{2}}\left|\bar{v}\left(\mathbf{q}_{i}\right)\right|^{2} \int d^{2} \mathbf{q}_{i}^{\prime} \delta^{2}\left(\mathbf{q}_{i}-\mathbf{q}_{i}^{\prime}\right) \cdots,
\end{aligned}
$$

where $|\bar{v}|^{2}$ is defined as the normalized distribution of momentum transfers from the scattering centers. In terms of Eq. (6), it is given by

$$
|\bar{v}(\mathbf{q})|^{2} \equiv \frac{1}{\sigma_{e l}} \frac{d^{2} \sigma_{e l}}{d^{2} \mathbf{q}}=\frac{1}{\pi} \frac{\mu_{e f f}^{2}}{\left(\mathbf{q}^{2}+\mu^{2}\right)^{2}}
$$

where in the Yukawa example, the normalization depends on the kinematic bounds through

$$
\frac{1}{\mu_{e f f}^{2}}=\frac{1}{\mu^{2}}-\frac{1}{\mathbf{q}_{\max }^{2}+\mu^{2}}
$$

and insures that $\int^{\mathbf{q}_{\max }} d^{2} \mathbf{q}|\bar{v}(\mathbf{q})|^{2}=1$. In numerical estimates we take $\mathbf{q}_{\text {max }}^{2}=s / 4 \approx 3 E \mu$. For asymptotic energies $\mu_{\text {eff }} \approx \mu$. Note also that unlike $v$, the barred $\bar{v}$ is independent of color factors and thus applies to both quark and gluon rescattering.

If the interaction with center $i$ is a double Born rather than a direct one, then the only change relative to (52) is that both $\mathbf{q}_{i}$ and $\mathbf{q}_{i}^{\prime}$ come with the same $( \pm i)^{2}=-1$ rather than the $(-i)(+i)=1$ factor and the phase shifts change relative sign. If the double Born interaction is in the amplitude, as in the example noted above, then the impact parameter average leads to 


$$
\begin{aligned}
\langle\cdots\rangle_{A_{\perp}} & =\left\langle\cdots \int \frac{d^{2} \mathbf{b}_{i}}{A_{\perp}}(-i) \int \frac{d^{2} \mathbf{q}_{i}}{(2 \pi)^{2}} v\left(0, \mathbf{q}_{i}\right) e^{-i \mathbf{q}_{i} \cdot \mathbf{b}_{i}}(-i) \int \frac{d^{2} \mathbf{q}_{i}^{\prime}}{(2 \pi)^{2}} v\left(0, \mathbf{q}_{i}^{\prime}\right) e^{-i \mathbf{q}_{i}^{\prime} \cdot \mathbf{b}_{i}} \cdots\right\rangle \\
& =\cdots(-1) \int \frac{d^{2} \mathbf{q}_{i}}{(2 \pi)^{2}} \frac{d^{2} \mathbf{q}_{i}^{\prime}}{(2 \pi)^{2}} \frac{(2 \pi)^{2} \delta^{2}\left(\mathbf{q}_{i}+\mathbf{q}_{i}^{\prime}\right)}{A_{\perp}} v\left(0, \mathbf{q}_{i}\right) v\left(0, \mathbf{q}_{i}^{\prime}\right) \cdots \\
& =\cdots(-1) \frac{\sigma}{A_{\perp}} \int \frac{d^{2} \mathbf{q}_{i}}{(2 \pi)^{2}}\left|\bar{v}\left(\mathbf{q}_{i}\right)\right|^{2} \int d^{2} \mathbf{q}_{i} \delta^{2}\left(\mathbf{q}_{i}+\mathbf{q}_{i}^{\prime}\right) \cdots
\end{aligned}
$$

The same holds true if the double Born is in the conjugate amplitude. The results above is the basis for the last graphical rule of the last section. A contribution to $n^{\text {th }}$ opacity involving $m$ "virtual" hits produces a factor $(-1)^{m}\left(\sigma / A_{\perp}\right)^{n}$.

A key to the analytic derivation in the next section is that the transverse impact parameter averages in Eqs. (52,55) diagonalize the products of amplitudes and complex conjugate amplitudes in the $\mathbf{q}_{i}, \mathbf{q}_{i}^{\prime}$ variables.

The question of which cross section $\sigma$ enters above depends on the details of the color algebra. At this point it is not at all clear since any particular contribution to a given order in opacity has an extremely complicated color structure in general [27]. However, as we prove in the next section the color algebra simplifies drastically in the sum over all possible real and virtual contributions [11, 13]. The color "triviality" of single inclusive distributions proved in the next section via the reaction operator approach, states that after summing all contributions, each integration over $\mathbf{q}_{i}$ is accompanied simply by a factor $\left(C_{A} C_{2}(T) / d_{A}\right)$. This implies that $\sigma=\sigma_{g}$ is the gluon elastic jet cross section that appears in both Eqs. (52,55), rather than the jet one $\left(\propto C_{R} C_{2}(T)\right)$. Recall that

$$
\sigma_{g}=\frac{C_{A}}{C_{R}} \sigma_{e l}(R, T)
$$

and hence

$$
C_{A} \lambda_{g}=C_{R} \lambda .
$$

Color triviality implies that the opacity expansion is in terms of powers of the gluon rather than the jet elastic cross section, i.e. $\propto\left(L / \lambda_{g}\right)^{n}$.

\section{RECURSIVE METHOD FOR OPACITY EXPANSION}

The inclusive gluon distribution to $\mathcal{O}\left((L / \lambda)^{1} \propto\left(\sigma_{e l} / A_{\perp}\right)^{1}\right)$ is a sum of $3^{2}$ direct and $2 \times 4$ virtual cut diagrams. If we want to proceed to second order in opacity $\mathcal{O}\left((L / \lambda)^{2} \propto\left(\sigma_{e l} / A_{\perp}\right)^{2}\right)$ there are $7^{2}$ direct plus $2 \times 86$ contributions. It is therefore useful to develop an recursive procedure for writing the sum of amplitudes in a certain class of diagrams. We will ignore the jet and elastic scattering parts of the diagrams which leaves us with simpler amplitudes similar to the ones discussed in Refs. [26, 27]. We recall the definitions of the Hard, Gunion-Bertsch and Cascade terms

$$
\begin{aligned}
\mathbf{H} & =\frac{\mathbf{k}}{\mathbf{k}^{2}}, & \mathbf{C}_{\left(i_{1} i_{2} \cdots i_{m}\right)}=\frac{\left(\mathbf{k}-\mathbf{q}_{i_{1}}-\mathbf{q}_{i_{2}}-\cdots-\mathbf{q}_{i_{m}}\right)}{\left(\mathbf{k}-\mathbf{q}_{i_{1}}-\mathbf{q}_{i_{2}}-\cdots-\mathbf{q}_{i_{m}}\right)^{2}}, \\
\mathbf{B}_{i} & =\mathbf{H}-\mathbf{C}_{i}, \quad & \mathbf{B}_{\left(i_{1} i_{2} \cdots i_{m}\right)\left(j_{1} j_{2} \cdots i_{n}\right)}=\mathbf{C}_{\left(i_{1} i_{2} \cdots j_{m}\right)}-\mathbf{C}_{\left(j_{1} j_{2} \cdots j_{n}\right)} .
\end{aligned}
$$

\section{A. Amplitude iteration}

There are two basic iteration steps that one has to consider to construct the inclusive distribution of gluon. The first one represents the addition of a "direct" interaction $D_{n}$ that changes the color or momentum of the target parton located at $z_{n}$ as illustrated by Fig. 2 . 

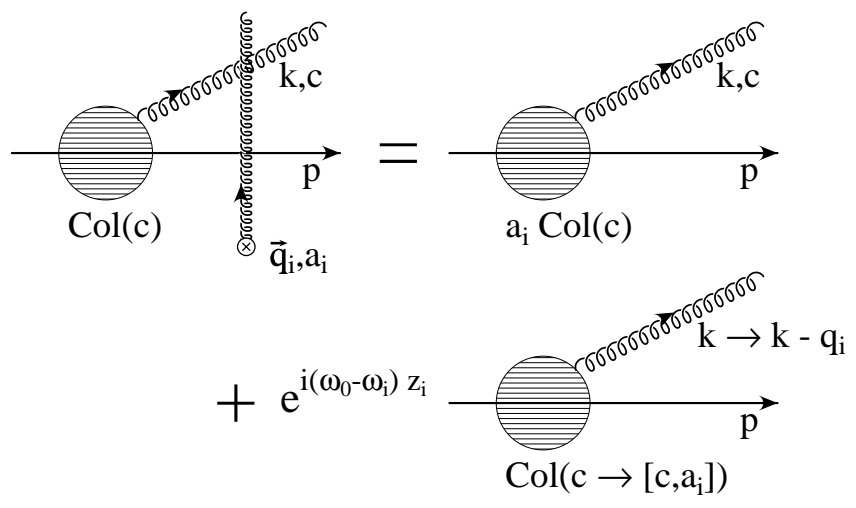

$-\left(-\frac{1}{2}\right)^{\mathrm{N}_{\mathrm{v}}} \mathrm{B}_{\mathrm{i}} \mathrm{e}^{\mathrm{i} \omega_{0} z_{\mathrm{i}}}\left[\mathrm{c}, \mathrm{a}_{\mathrm{i}}\right] \mathrm{T}_{\mathrm{el}}$

FIG 2. Diagrammatic representation of the sum of amplitudes generated by $\mathcal{A} D_{i}=\mathcal{A} X_{i, 0}+\mathcal{A} X_{i, 1}+$ $\mathcal{A} G^{-1} X_{i, 0} G_{i}$.

The second iteration step corresponds to a double Born "virtual" interaction $V_{n}$ that leaves both the color and momentum of the target parton unchanged and is illustrated in Fig. 3.

Our goal here is to construct operators $\hat{D}_{n}, \hat{V}_{n}$ that can be used to construct recursively the new amplitudes.

It is important to note that each new diagram produces a difference of two phase factors with the exception of one special diagram, $\left(\mathcal{A} D_{n}\right)_{0} \equiv \mathcal{A} G^{-1} X_{n, 0} G_{n}$, corresponding to the emission of the gluon after, $z_{n}$. The upper limits of emission is $z=\infty$ in that case, and only the lower bound phase factor, $-e^{i \omega_{0} z_{n}}$ survives on account of the adiabatic damping factor $\exp (-\epsilon|z|)$. Therefore all but one diagram can be divided into two parts which can then be recombined into more easily interpretable Hard, Gunion-Bertsch and Cascade amplitudes (58) as in [26, 27].

The sum of amplitudes in class $\mathcal{A}$ can be denoted by

$$
\mathcal{A}(x, \mathbf{k}, c) \equiv \sum_{\alpha} \mathcal{A}_{\alpha}(x, \mathbf{k}) \operatorname{Col}(c)_{\alpha}
$$

where $\mathcal{A}_{\alpha}(x, \mathbf{k})$ represents the kinematical part, $\operatorname{Col}(c)_{\alpha}$ stands for the color matrix for the distinct graphs in this class enumerated by $\alpha$. There is of course considerable freedom in rearranging the terms of this sum. Since by definition classes are constructed by repeated operations of either of one of three operations, $\hat{\mathbf{1}}, \hat{D}_{i}, \hat{V}_{i}$ it is convenient to enumerate the $3^{n}$ different classes of diagrams via a tensor notation, $\mathcal{A}_{i_{1} \cdots i_{n}}$, where the indices $i_{j}=0,1,2$ specify whether there was no, a direct, or a virtual interaction with the target parton at $z_{j}$. The sum of amplitudes for class, $\mathcal{A}_{i_{1} \cdots i_{n}}$, can be written explicitly in the form

$$
\mathcal{A}_{i_{1} \cdots i_{n}}(x, \mathbf{k}, c)=\prod_{m=1}^{n}\left(\delta_{0, i_{m}}+\delta_{1, i_{m}} \hat{D}_{m}+\delta_{2, i_{m}} \hat{V}_{m}\right) G_{0}(x, \mathbf{k}, c)
$$

Here $G_{0}$ is the color matrix amplitude that corresponds to the initial source or kernel of the jet and gluon in the limit of no final state interactions.

The inclusive induced "probability" distribution at order $n$ in the opacity expansion can then be computed from the following sum of products over the $3^{n}$ classes that contribute at that order:

$$
P_{n}(x, \mathbf{k})=\overline{\mathcal{A}}^{i_{1} \cdots i_{n}}(c) \mathcal{A}_{i_{1} \cdots i_{n}}(c) \equiv \operatorname{Tr} \sum_{i_{1}=0}^{2} \cdots \sum_{i_{n}=0}^{2} \overline{\mathcal{A}}_{i_{1} \cdots i_{n}}^{\dagger}(x, \mathbf{k}, c) A_{i_{1} \cdots i_{n}}(x, \mathbf{k}, c),
$$

where the unique complementary class that contracts with $\mathcal{A}_{i_{1} \cdots i_{n}}$ is defined by 


$$
\begin{aligned}
& \overline{\mathcal{A}}_{i_{1} \cdots i_{n}}(x, \mathbf{k}, c) \equiv \prod_{m=1}^{n}\left(\delta_{0, i_{m}} \hat{V}_{m}+\delta_{1, i_{m}} \hat{D}_{m}+\delta_{2, i_{m}}\right) G_{0}(x, \mathbf{k}, c) \\
& \overline{\mathcal{A}}^{i_{1} \cdots i_{n}}(x, \mathbf{k}, c) \equiv G_{0}^{\dagger}(x, \mathbf{k}, c) \prod_{m=1}^{n}\left(\delta_{0, i_{m}} \hat{V}_{m}^{\dagger}+\delta_{1, i_{m}} \hat{D}_{m}^{\dagger}+\delta_{2, i_{m}}\right)
\end{aligned}
$$

For example, $\overline{\mathcal{A}}_{0210}=\hat{D}_{3} \hat{V}_{2} G_{0}$ for $\mathcal{A}_{2012}=\hat{V}_{4} \hat{D}_{3} \hat{V}_{1} G_{0}$. Note that $\overline{\mathcal{A}} \mathcal{A}$ includes products of classes and complementary classes with different powers of the external coupling, $g_{s}$, but every product in the sum is the same order, $\alpha_{s}^{2 n+1}$. These mixed terms are the unitarity corrections to the direct $\overline{\mathcal{A}}^{1 \cdots 1} \mathcal{A}_{1 \cdots 1}$ term that contribute to inclusive processes when the target recoils are not tagged, i.e. measured exclusively. Note that $P_{n}$ is not positive definite except for $n=0$, and we refer to it as a "probability" only figuratively.

With this tensor classification and construction, it becomes possible to construct $P_{n}$ recursively from lower rank (opacity) classes through the insertion of a "reaction" operator as follows:

$$
P_{n}=\overline{\mathcal{A}}^{i_{1} \cdots i_{n-1}} \hat{R}_{n} \mathcal{A}_{i_{1} \cdots i_{n-1}}, \quad \hat{R}_{n}=\hat{D}_{n}^{\dagger} \hat{D}_{n}+\hat{V}_{n}+\hat{V}_{n}^{\dagger} .
$$

We emphasize that it is possible to write the inclusive probability in this simple form only because the transverse impact parameter averages in Eqs. (52,55) diagonalize the products of amplitudes and complementary conjugate amplitudes in the $\mathbf{q}_{i}, \mathbf{q}_{i}^{\prime}$ variables. If the transverse area of the target were not large in comparison to the cross section, then off diagonal components in those variables would appear that would have to folded with a suitable transverse Glauber profile functions as discussed in [ [6]. The simple algebraic structure of the problem in Eq. (63) therefore hinges on the validity of the transverse averaging via Eqs. (52,55).

To construct $\hat{D}_{n}$, consider first the contribution $\mathcal{A} X_{n, 0}$ from Eq. (40). In this case, a new direct interaction is attached to the jet line at $z_{n}$. For the partial sum of amplitudes in $\mathcal{A}_{i_{1} \cdots i_{n-1}}$ corresponding to emitting the gluon before the last real or virtual interaction at $z_{f} \leq z_{n-1}$, the direct interaction of the jet at $z_{n}$ simply multiplies that partial sum by $a_{n}$. No extra phases are introduced since the energy of the jet is assumed to be very high. As noted before, for every class, there is a term in $\mathcal{A}_{i_{1} \cdots i_{n-1}}$ corresponding to a graph where the gluon is emitted after the last interaction point $z_{f}$. That term has the explicit form

$$
\left(-\frac{1}{2}\right)^{N_{v}\left(\mathcal{A}_{i_{1} \cdots i_{n-1}}\right)} \mathbf{H}\left(-e^{i \omega_{0} z_{f}}\right) c T_{e l}\left(\mathcal{A}_{i_{1} \cdots i_{n-1}}\right),
$$

where the elastic color matrices for that class and its complementary are denoted by

$$
T_{e l}\left(\mathcal{A}_{i_{1} \cdots i_{n-1}}\right) \equiv\left(a_{n-1}\right)^{i_{n-1}} \cdots\left(a_{1}\right)^{i_{1}}, \quad T_{e l}^{\dagger}\left(\overline{\mathcal{A}}^{i_{1} \cdots i_{n-1}}\right) \equiv\left(a_{1}\right)^{2-i_{1}} \cdots\left(a_{n-1}\right)^{2-i_{n-1}} .
$$

For example, $T_{e l}\left(\mathcal{A}_{1021}=\hat{D}_{4} \hat{V}_{3} \hat{D}_{1} G_{0}\right)=a_{4}\left(a_{3} a_{3}\right) a_{1}=C_{R} a_{4} a_{1}$. Eq. (65) implies the following identity holds for elastic color factors:

$$
T_{e l}^{\dagger}\left(\overline{\mathcal{A}}^{i_{1} \cdots i_{n-1}}\right) T_{e l}\left(\mathcal{A}_{i_{1} \cdots i_{n-1}}\right)=C_{R}^{n-1}
$$

valid for all $3^{n-1}$ components of rank $n-1$ classes.

The factor $\left(-\frac{1}{2}\right)^{N_{v}}$ in Eq. (64) arises because every virtual contact interaction in the class acquires a factor $-\frac{1}{2}$ from the the contact limit of the contour integration over longitudinal momentum (see Sec. II and the Appendix). The numbers, $N_{v}, \bar{N}_{v}$, of such contact interactions in class, $\mathcal{A}_{i_{1} \cdots i_{n-1}}$, and its complementary class, $\overline{\mathcal{A}}^{i_{1} \cdots i_{n-1}}$, are

$$
N_{v}=N_{v}\left(\mathcal{A}_{i_{1} \cdots i_{n-1}}\right)=\sum_{m=1}^{n-1} \delta_{2, i_{m}}, \quad \bar{N}_{v}=N_{v}\left(\overline{\mathcal{A}}^{i_{1} \cdots i_{n-1}}\right)=\sum_{m=1}^{n-1} \delta_{0, i_{m}} .
$$

For example $N_{v}=2, \bar{N}_{v}=2$ for $\mathcal{A}_{01202}=\hat{V}_{5} \hat{V}_{3} \hat{D}_{2} G_{0}$. A useful bookkeeping identity that we will need is

$$
\sum_{i_{1}, \cdots, i_{m}}\left(-\frac{1}{2}\right)^{N_{v}\left(\overline{\mathcal{A}}_{i_{1} \cdots i_{m}}\right)}\left(-\frac{1}{2}\right)^{N_{v}\left(\mathcal{A}_{i_{1} \cdots i_{m}}\right)}=\left(-\frac{1}{2}-\frac{1}{2}+1\right)^{m}=0 .
$$

Now consider how a jet interaction at $z_{n}$ changes the graph Eq. (64). Besides being multiplied by $a_{n}$, the only other change is that the phase factor changes into a difference of phase factors as 


$$
-e^{i \omega_{0} z_{f}} \stackrel{X_{n, 0}}{\longrightarrow} e^{i \omega_{0} z_{n}}-e^{i \omega_{0} z_{f}}
$$

The part, $\propto e^{i \omega_{0} z_{f}}$, on the right hand side can be added back to the sum of all the other graphs with emission before $z_{f}$. The modified sum $a_{n} \mathcal{A}_{i_{1} \cdots i_{n-1}}$ therefore includes this part of Eq. (69). In summary, the component of the $\hat{D}_{n}$ operator that takes into account the jet rescattering at $z_{n}$ is simply multiplication by the color matrix, $a_{n}$, in the jet representation. This then identifies the first part of the $\hat{D}_{n}$ operator as

$$
\hat{D}_{n}=\hat{\mathbf{1}} a_{n}+\cdots .
$$

The left over part $\propto e^{i \omega_{0} z_{n}}$ of Eq. (69) can be combined with the graph $\left(\mathcal{A} G^{-1} X_{n, 0} G_{n}\right)$, e.g., $M_{1,1,0}, M_{2,2,0}$ in Appendix A and $\mathrm{C}$, that accounts for gluon emission after $z_{n}$. This combination leads to the following extra term in $\mathcal{A}_{i_{1} \cdots i_{n}}$ :

$$
-\left(-\frac{1}{2}\right)^{N_{v}\left(\mathcal{A}_{i_{1} \cdots i_{n-1}}\right)} \mathbf{H} e^{i \omega_{0} z_{n}}\left[c, a_{n}\right] T_{e l}\left(\mathcal{A}_{i_{1} \cdots i_{n-1}}\right) .
$$

The sum of graphs $\mathcal{A}\left(X_{n, 0}+G^{-1} X_{n, 0} G_{n}\right)$ is therefore $a_{n} \mathcal{A}_{i_{1} \cdots i_{n}}$ plus Eq. (71).

To finish constructing $\hat{D}_{n}$, we need to consider the effect of an elastic scattering of the gluon produced at an earlier point in the medium. The gluon "cascade" interaction graph $\mathcal{A} X_{n, 1}$ (see e.g., $M_{2,0,3}$ in Appendix B) introduces the following changes in the amplitude: First an extra phase shift $\exp \left[i\left(\omega_{0}-\omega_{n}\right) z_{n}\right]$ arises due to the difference of gluon energies before and after the transverse momentum exchange, $\mathbf{q}_{n}$, at $z_{n}$. This is in contrast to the jet rescattering where that energy change was negligible. Second, the gluon color is rotated by $c \rightarrow\left[c, a_{n}\right]$. Finally, the transverse momentum in $\mathcal{A}(x, \mathbf{k}, c)$ is shifted backward by $\mathbf{k} \rightarrow \mathbf{k}-\mathbf{q}_{n}$ in order that same final state $\mathbf{k}$ is reached. This part of the $\hat{D}_{n}$ operator therefore simply transforms

$$
\mathcal{A}_{i_{1} \cdots i_{n-1}}(\mathbf{k}, c) \stackrel{X_{n, 1}}{\longrightarrow} \hat{S}_{n} \mathcal{A}_{i_{1} \cdots i_{n-1}}=e^{i\left(\omega_{0}-\omega_{n}\right) z_{n}} \mathcal{A}_{i_{1} \cdots i_{n-1}}\left(\mathbf{k}-\mathbf{q}_{n},\left[c, a_{n}\right]\right) .
$$

The "shift" or gluon scattering operator that implements gluon rescattering at $z_{n}$ is thus defined by

$$
\hat{S}_{n}=i f^{c a_{n} d} \times e^{i\left(\omega_{0}-\omega_{n}\right) z_{n}} e^{i \mathbf{q}_{n} \cdot \hat{\mathbf{b}}} .
$$

Here, $\hat{\mathbf{b}} \equiv i \overrightarrow{\nabla_{\mathbf{k}}}$ is the impact parameter operator conjugate to $\mathbf{k}$ such that

$$
e^{i \mathbf{q} \cdot \hat{\mathbf{b}}} f(\mathbf{k})=f(\mathbf{k}-\mathbf{q}) .
$$

This transverse momentum shift together with a class independent phase shift and a color rotation (via the structure constants $f^{a b c}$ ) enlarges $\mathcal{A}_{i_{1} \cdots i_{n-1}}$ to include all but one of the gluon final state interaction diagrams with an interaction at $z_{n}$.

The left over graph not included in $(72)$ is the one where the jet scatters elastically up to previous last interaction point $z_{f}$, emits a gluon with transverse momentum $\mathbf{k}-\mathbf{q}_{n}$ between $z_{f}$ and $z_{n}$, and the gluon scatters at $z_{n}$ (see e.g., $M_{1,0,1}$ in Appendix A). The integral over the emission interval leaves a characteristic differences of phases $e^{i \omega_{n} z_{n}}-e^{i \omega_{n} z_{f}}$, while the elastic scattering introduces a phase shift factor $e^{i\left(\omega_{0}-\omega_{n}\right) z_{n}}$ as well. The kinematic part of this cascade amplitude is $\mathbf{C}_{n}$. The part proportional to $e^{i \omega_{n} z_{n}}$ contributes therefore the following extra term

$$
+\left(-\frac{1}{2}\right)^{N_{v}} \mathbf{C}_{n} e^{i\left(\omega_{0}-\omega_{1}\right) z_{n}} e^{i \omega_{1} z_{n}}\left[c, a_{n}\right] T_{e l}\left(\mathcal{A}_{i_{1} \cdots i_{n-1}}\right),
$$

where again $N_{v}$ is the number of contact interactions before $z_{n}$. Recalling that $\mathbf{B}_{n}=\mathbf{H}-\mathbf{C}_{n}$ has the physical interpretation of a Gunion-Bertsch gluon source term at $z_{n}$, the two extra terms from Eq. (74) and Eq. (71) can be conveniently combined to form

$$
\begin{aligned}
\hat{B}_{n} \mathcal{A}_{i_{1} \cdots i_{n-1}}(c) & \equiv-\left(-\frac{1}{2}\right)^{N_{v}\left(\mathcal{A}_{i_{1} \cdots i_{n-1}}\right)} i f^{c a_{n} d} \times \mathbf{B}_{n} e^{i \omega_{0} z_{n}} d T_{e l}\left(\mathcal{A}_{i_{1} \cdots i_{n-1}}\right) \\
& =-\prod_{m=1}^{n-1}\left(-\frac{1}{2}\right)^{\delta_{2, i_{m}}} \mathbf{B}_{n} e^{i \omega_{0} z_{n}}\left[c, a_{n}\right] a_{n-1}^{i_{n-1}} \cdots a_{1}^{i_{1}}
\end{aligned}
$$

Combination of three steps $X_{n, 0}+X_{n, 1}+G^{-1} X_{n, 0} G$ can therefore be summarized by the following operation on rank $n-1$ class elements: 


$$
\begin{aligned}
\hat{D}_{n} \mathcal{A}_{i_{1} \cdots i_{n-1}}(x, \mathbf{k}, c) \equiv & \left(a_{n}+\hat{S}_{n}+\hat{B}_{n}\right) \mathcal{A}_{i_{1} \cdots i_{n-1}}(x, \mathbf{k}, c) \\
= & a_{n} \mathcal{A}_{i_{1} \cdots i_{n-1}}(x, \mathbf{k}, c)+e^{i\left(\omega_{0}-\omega_{n}\right) z_{n}} \mathcal{A}_{i_{1} \cdots i_{n-1}}\left(x, \mathbf{k}-\mathbf{q}_{n},\left[c, a_{n}\right]\right) \\
& -\left(-\frac{1}{2}\right)^{N_{v}\left(\mathcal{A}_{i_{1} \cdots i_{n-1}}\right)} \mathbf{B}_{n} e^{i \omega_{0} z_{n}}\left[c, a_{n}\right] T_{e l}\left(\mathcal{A}_{i_{1} \cdots i_{n-1}}\right)
\end{aligned}
$$

We note that the common factor to all diagrams $2 i g_{s} \epsilon \cdot(\ldots)$ is suppressed for clarity.

We now turn to the second class of diagrams, $\hat{V}_{n} \mathcal{A}_{i_{1} \cdots i_{n-1}}$ created by inserting contact interaction at $z_{n}$. This new class is constructed via Eq. (41) and illustrated in Fig. 3.
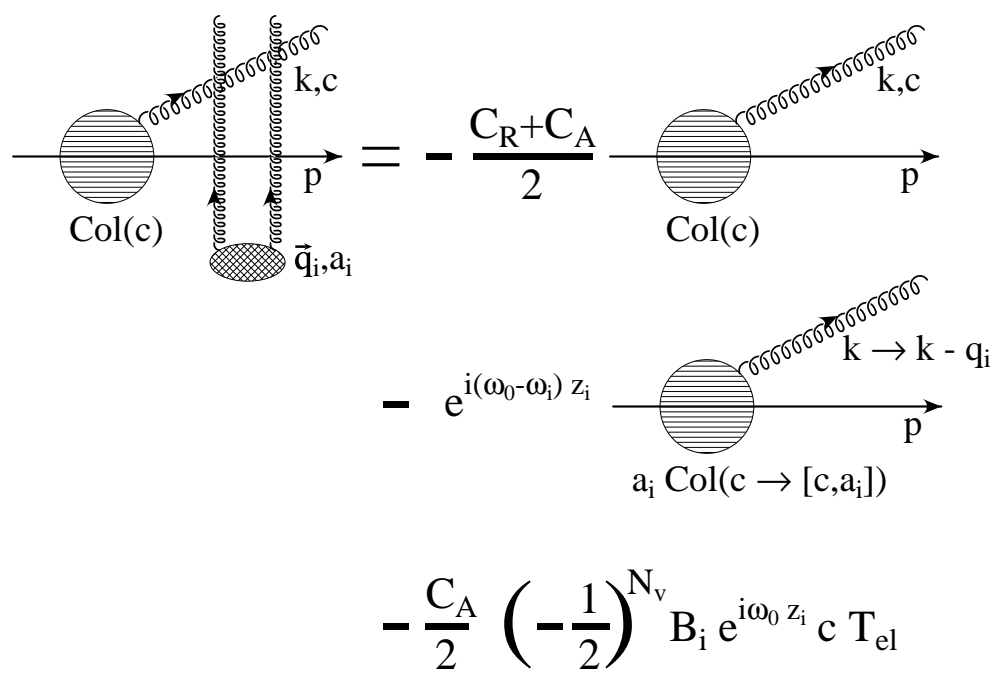

FIG 3. Diagrammatic representation of the sum of amplitudes, generated by $\mathcal{A} V_{i}=\mathcal{A} O_{i, 0}+\mathcal{A} O_{i, 1}+$ $\mathcal{A} O_{i, 2}+\mathcal{A} G^{-1} O_{i, 0} G_{i}$.

For a double Born interaction at $z_{n}$, the transverse coordinate integration can be performed before multiplying by a complementary hermitian conjugate amplitudes since the coordinates of a virtual interaction cannot occur in the complementary class. The transverse coordinate integration on the center of the $i^{\text {th }}$ interaction enforces (see Eq. (19)) that the net momentum transfered in the two legs vanishes via a factor $\delta^{2}\left(\mathbf{q}_{i}+\mathbf{q}_{i}^{\prime}\right)$.

Consider first the sum of graphs where the two legs of the contact interaction are attached to the jet line $\left(\mathcal{A} O_{n, 0}\right)$ (see,e.g. $M_{2,0,0}^{c}$ in Appendix C). The situation is similar to Eq. (70) except that two color factors $a_{n} a_{n}=C_{R}$ multiply the rank $n-1$ amplitudes instead of one. In addition a factor of $-\frac{1}{2}$ appears due to the contact limit of the contour integration. Thus, this part of the virtual interaction simply transforms

$$
\mathcal{A}_{i_{1} \cdots i_{n-1}} \stackrel{O_{n, 0}}{\longrightarrow}-\frac{C_{R}}{2} \mathcal{A}_{i_{1} \cdots i_{n-1}} .
$$

The second term, $\mathcal{A} O_{n, 1}$ in Eq. (41) is the contact with the gluon at $z_{n}$ (see $M_{2,0,3}^{c}$ in Appendix B). Since $i f^{c a_{n} d} i f^{d a_{n} e}=C_{A} \delta_{c, e}$, the color structure of this term is also trivial. In addition, since the total momentum transfer is now zero, no extra phase factor arises and the gluon momentum in $\mathcal{A}_{i_{1} \cdots i_{n-1}}$ remains unchanged. The contact gluon interaction at $z_{n}$ therefore contributes

$$
\mathcal{A}_{i_{1} \cdots i_{n-1}} \stackrel{O_{n, 1}}{\longrightarrow}-\frac{C_{A}}{2} \mathcal{A}_{i_{1} \cdots i_{n-1}} .
$$

The mixed contact interaction $\mathcal{A O}_{i, 2}$ (see, e.g. $M_{2,0,1}^{c}$ in Appendix D) is the most subtle. Even though the net momentum transfer vanishes, in this case both the jet and gluons receive (opposite) non-vanishing 
transverse momentum transfers, $\pm \mathbf{q}_{n}$. This leads to a highly non-local modification of $\mathcal{A}_{i_{1} \cdots i_{n-1}}$. The contact limit of the longitudinal momentum transfer contour integration produces in this case a factor of -1 as shown in the Appendix. The elastic scattering of the gluon produces multiplicative phase factor, $e^{i\left(\omega_{0}-\omega_{n}\right) z_{n}}$. The jet elastic scattering introduces no new phase in the small $x$ limit as before. The transverse momentum exchange, to the gluon requires that $\mathbf{k}$ is again shifted back by $\mathbf{q}_{n}$ as in Eq. (72). The gluon color is also rotated as in (72). Furthermore, the scattering of the jet introduces another color matrix $a_{n}$. This mixed contact interaction therefore simply produces the following variant of (72):

$$
\mathcal{A}_{i_{1} \cdots i_{n-1}}(\mathbf{k}, c) \stackrel{O_{n, 2}}{\longrightarrow} a_{n} \hat{S}_{n} \mathcal{A}_{i_{1} \cdots i_{n-1}}=e^{i\left(\omega_{0}-\omega_{n}\right) z_{n}} a_{n} \mathcal{A}_{i_{1} \cdots i_{n-1}}\left(\mathbf{k}-\mathbf{q}_{n},\left[c, a_{n}\right]\right)
$$

with again one extra term that can be recombined with the amplitude of gluon radiation after the contact interaction at $z_{n}$ to form a virtual Gunion-Bertsch source

$$
-a_{n} \hat{B}_{n} \mathcal{A}_{i_{1} \cdots i_{n-1}}=-\left(-\frac{1}{2}\right)^{N_{v}\left(\mathcal{A}_{i_{1} \cdots i_{n-1}}\right)} \frac{C_{A}}{2} \mathbf{B}_{n} e^{i \omega_{0} z_{n}} c T_{e l}\left(\mathcal{A}_{i_{1} \cdots i_{n-1}}\right),
$$

where we used $a_{n}\left[c, a_{n}\right]=-\frac{1}{2} C_{A} c$ to simplify the color algebra.

The double Born interaction at $z_{n}$ can therefore be implemented by the following operator

$$
\hat{V}_{n}=-\frac{1}{2}\left(C_{A}+C_{R}\right)-a_{n} \hat{S}_{n}-a_{n} \hat{B}_{n}=-a_{n} \hat{D}_{n}-\frac{1}{2}\left(C_{A}-C_{R}\right) .
$$

Specifically, the virtual iteration of a rank $n-1$ class gives

$$
\begin{aligned}
\hat{V}_{n} \mathcal{A}_{i_{1} \cdots i_{n-1}}(x, \mathbf{k}, c)= & -\frac{C_{R}+C_{A}}{2} \mathcal{A}_{i_{1} \cdots i_{n-1}}(x, \mathbf{k}, c)-e^{i\left(\omega_{0}-\omega_{n}\right) z_{n}} a_{n} \mathcal{A}_{i_{1} \cdots i_{n-1}}\left(x, \mathbf{k}-\mathbf{q}_{n},\left[c, a_{n}\right]\right) \\
& -\left(-\frac{1}{2}\right)^{N_{v}} \frac{C_{A}}{2} \mathbf{B}_{n} e^{i \omega_{0} z_{n}} c a_{n-1}^{i_{n-1}} \cdots a_{1}^{i_{1}} .
\end{aligned}
$$

\section{B. Reaction operator recursion to all orders in opacity}

We emphasize that this recursive process is a completely general and applies both the hard emission kernel $\operatorname{Ker}^{(\mathrm{H})}$ Eq. (39) and the asymptotic Gunion-Bertsch $\operatorname{Ker}^{(\mathrm{GB})}$ Eq. (38).

The key to unraveling the interplay between direct and virtual interaction is the operator identity Eq. (81) between $\hat{D}_{n}$ and $\hat{V}_{n}$. This makes it possible to relate the $n^{\text {th }}$ order in opacity gluon emission "probability" distribution $P_{n}$ to the probability at $(n-1)^{\text {th }}$ order by expressing the reaction operator $\hat{R}_{n}$ in Eq. (63) as

$$
\hat{R}_{n}=\left(\hat{D}_{n}-a_{n}\right)^{\dagger}\left(\hat{D}_{n}-a_{n}\right)-C_{A}=\left(\hat{S}_{n}+\hat{B}_{n}\right)^{\dagger}\left(\hat{S}_{n}+\hat{B}_{n}\right)-C_{A} .
$$

A further major simplification occurs because both $\hat{S}$ and $\hat{B}$ involve the same gluon color rotation through $i f^{c a_{n} d}$. We show next how this unravels the color algebra and reduces it to simply powers of $C_{A}$ and $C_{R}$. We therefore prove color "triviality" of the reaction operator, a property that is implicit in the work of Refs. [ 11, 13] and which has been proved in a somewhat more involved way via path integral techniques in [ 34] for quark jets only.

First, we note that the shift operator has the property that

$$
\hat{S}_{n}^{\dagger} \hat{S}_{n}=C_{A} e^{-\mathbf{q}_{n} \overleftarrow{\nabla_{\mathbf{k}}}} e^{-\mathbf{q}_{n} \vec{\nabla}_{\mathbf{k}}}
$$

Therefore $\hat{S}^{\dagger} \hat{S}$ is diagonal in color space. This allows us to compute the effect of an extra gluon cascade interactions through simple transverse momentum shifts

$$
\begin{aligned}
\overline{\mathcal{A}}^{i_{1} \cdots i_{n-1}}\left(\hat{S}_{n}^{\dagger} \hat{S}_{n}-C_{A}\right) \mathcal{A}_{i_{1} \cdots i_{n-1}} & =C_{A}\left(\overline{\mathcal{A}}^{i_{1} \cdots i_{n-1}}\left(\mathbf{k}-\mathbf{q}_{n}, c\right) \mathcal{A}_{i_{1} \cdots i_{n-1}}\left(\mathbf{k}-\mathbf{q}_{n}, c\right)-\overline{\mathcal{A}}^{i_{1} \cdots i_{n-1}}(\mathbf{k}, c) \mathcal{A}_{i_{1} \cdots i_{n-1}}(\mathbf{k}, c)\right) \\
& =C_{A}\left(P_{n-1}\left(\mathbf{k}-\mathbf{q}_{n}\right)-P_{n-1}(\mathbf{k})\right)=C_{A}\left(e^{i \mathbf{q}_{n} \cdot \hat{\mathbf{b}}}-1\right) P_{n-1}(\mathbf{k}) .
\end{aligned}
$$

Next, consider the modulus square of the extra Gunion-Bertsch source for two or more scatterings of the jet+gluon system $(n>1)$ : 


$$
\begin{aligned}
\overline{\mathcal{A}}^{i_{1} \cdots i_{n-1}} \hat{B}_{n}^{\dagger} \hat{B}_{n} \mathcal{A}_{i_{1} \cdots i_{n-1}} & =C_{A}\left|\mathbf{B}_{n}\right|^{2} \sum_{i_{1}, \cdots, i_{n-1}}\left(-\frac{1}{2}\right)^{\bar{N}_{v}}\left(-\frac{1}{2}\right)^{N_{v}} T_{e l}^{\dagger}\left(\overline{\mathcal{A}}^{i_{1} \cdots i_{n-1}}\right) c c T_{e l}\left(\mathcal{A}_{i_{1} \cdots i_{n-1}}\right) \\
& =C_{A} C_{R}^{n}\left|\mathbf{B}_{n}\right|^{2} \sum_{i_{1}, \cdots, i_{n-1}} \prod_{m=1}^{n-1}\left(-\frac{1}{2}\right)^{\delta_{2, i_{m}}}\left(-\frac{1}{2}\right)^{\delta_{0, i_{m}}}(1)^{\delta_{1, i_{m}}}=0
\end{aligned}
$$

where we used Eq. (66), and the bookkeeping identity (68). Therefore, the virtual corrections cancel the direct extra Gunion-Bertsch source term for $n>1$. For the special case with $n=1$, however, this diagonal term survives

$$
G_{0}^{\dagger} \hat{B}_{1}^{\dagger} \hat{B}_{1} G_{0}=C_{A} C_{R}\left|\mathbf{B}_{1}\right|^{2} .
$$

Finally, we have to calculate the interference between the cascade and Gunion-Bertsch source terms in (63). The two gluon color factor $f^{c a_{n} d}$ in Eqs. 73 75) again contract to form a color diagonal factor $C_{A}$. This leaves

$$
2 \operatorname{Re} \overline{\mathcal{A}}^{i_{1} \cdots i_{n-1}} \hat{B}_{n}^{\dagger} \hat{S}_{n} \mathcal{A}_{i_{1} \cdots i_{n-1}}=-2 C_{A} \mathbf{B}_{n} \cdot\left(\operatorname{Re} e^{-i \omega_{n} z_{n}} e^{i \mathbf{q}_{\mathbf{n}} \cdot \hat{\mathbf{b}}} \mathbf{I}_{n-1}\right),
$$

where the $\mathbf{I}_{n-1}$ term in the iteration step is easily seen to be

$$
\begin{aligned}
\mathbf{I}_{n-1} \equiv & \sum_{i_{1} \cdots i_{n-1}}\left(-\frac{1}{2}\right)^{N_{v}\left(\overline{\mathcal{A}}^{i_{1} \cdots i_{n-1}}\right)} T_{e l}^{\dagger}\left(\overline{\mathcal{A}}^{i_{1} \cdots i_{n-1}}\right) c \mathcal{A}_{i_{1} \cdots i_{n-1}} \\
= & \sum_{i_{1} \cdots i_{n-2}}\left(-\frac{1}{2}\right)^{N_{v}\left(\overline{\mathcal{A}}^{i_{1} \cdots i_{n-2}}\right)} T_{e l}^{\dagger}\left(\overline{\mathcal{A}}^{i_{1} \cdots i_{n-2}}\right) \\
& \times \sum_{i_{n-1}=0}^{2}\left\{\left(-\frac{1}{2}\right)^{\delta_{0, i_{n-1}}} a_{n-1}^{2-i_{n-1}}\right\} c\left(\delta_{0, i_{n-1}}+\delta_{1, i_{n-1}} \hat{D}_{n-1}+\delta_{2, i_{n-1}} \hat{V}_{n-1}\right) \mathcal{A}_{i_{1} \cdots i_{n-2}} \\
= & \sum_{i_{1} \cdots i_{n-2}}\left(-\frac{1}{2}\right)^{N_{v}\left(\overline{\mathcal{A}}_{i_{1} \cdots i_{n-2}}\right)} T_{e l}^{\dagger}\left(\overline{\mathcal{A}}_{i_{1} \cdots i_{n-2}}\right)\left(-\frac{1}{2} a_{n-1} a_{n-1} c+a_{n-1} c \hat{D}_{n-1}+c \hat{V}_{n-1}\right) \mathcal{A}_{i_{1} \cdots i_{n-2}} .
\end{aligned}
$$

This contribution builds up on the class of diagrams $\mathcal{A}_{i_{1} \cdots i_{n-2}}$ under the action of an iteration operator which can be reduced using Eqs. (73,81) into the form

$$
\begin{aligned}
-\frac{1}{2} a_{n} a_{n} c+a_{n} c \hat{D}_{n}+c \hat{V}_{n} & =\left[a_{n}, c\right] \hat{D}_{n}-\frac{C_{A}}{2} c=\left[a_{n}, c\right]\left(\hat{S}_{n}+\hat{B}_{n}\right)-C_{A} c \\
& =C_{A}\left(e^{i\left(\omega_{0}-\omega_{n}\right) z_{n}} e^{i \mathbf{q}_{n} \cdot \hat{\mathbf{b}}}-1\right) c+\left[a_{n}, c\right] \hat{B}_{n} .
\end{aligned}
$$

Next, we note that the contributions from $\hat{B}$ actually cancel exactly for $n>2$ in the sum over all classes due to the bookkeeping identity:

$$
\begin{aligned}
& \sum_{i_{1} \cdots i_{n-2}}\left(-\frac{1}{2}\right)^{N_{v}\left(\overline{\mathcal{A}}^{i_{1} \cdots i_{n-2}}\right)} T_{e l}^{\dagger}\left(\overline{\mathcal{A}}^{i_{1} \cdots i_{n-2}}\right)\left[a_{n-1}, c\right] \hat{B}_{n-1} \mathcal{A}_{i_{1} \cdots i_{n-2}} \\
= & -C_{A} C_{R} \mathbf{B}_{n-1} e^{i \omega_{0} z_{n-1}} \sum_{i_{1} \cdots i_{n-2}}\left(-\frac{1}{2}\right)^{N_{v}\left(\overline{\mathcal{A}}_{i_{1} \cdots i_{n-2}}\right)}\left(-\frac{1}{2}\right)^{N_{v}\left(\mathcal{A}_{i_{1} \cdots i_{n-2}}\right)} T_{e l}^{\dagger}\left(\overline{\mathcal{A}}_{i_{1} \cdots i_{n-2}}\right) T_{e l}\left(\mathcal{A}_{i_{1} \cdots i_{n-2}}\right)=0 .
\end{aligned}
$$

For the special case with $n=2$ we can evaluate $\mathbf{I}_{1}$ directly from the definition Eq. (89) and the initial hard amplitude to obtain

$$
\begin{aligned}
\mathbf{I}_{1} & =C_{A}\left(e^{i\left(\omega_{0}-\omega_{1}\right) z_{1}} e^{i \mathbf{q}_{1} \cdot \hat{\mathbf{b}}}-1\right) c G_{0}+\left[a_{1}, c\right] \hat{B}_{1} G_{0}=-C_{A} C_{R}\left(\left(e^{i\left(\omega_{0}-\omega_{1}\right) z_{1}} e^{i \mathbf{q}_{1} \cdot \hat{\mathbf{b}}}-1\right) \mathbf{H} e^{i \omega_{0} z_{0}}+\mathbf{B}_{1} e^{i \omega_{0} z_{1}}\right) \\
& =-C_{A} C_{R}\left(e^{i\left(\omega_{0}-\omega_{1}\right) z_{1}} e^{i \mathbf{q}_{1} \cdot \hat{\mathbf{b}}}-1\right) \mathbf{H}\left(e^{i \omega_{0} z_{0}}-e^{i \omega_{0} z_{1}}\right) .
\end{aligned}
$$


In the last line the Gunion-Bertsch amplitude was rewritten with the help of the shift operator Eq. (73) as being derived from the hard vertex kernel

$$
\mathbf{B}_{1} e^{i \omega_{0} z_{1}}=-\left(e^{i\left(\omega_{0}-\omega_{1}\right) z_{1}} e^{i \mathbf{q}_{1} \cdot \hat{\mathbf{b}}}-1\right) \mathbf{H} e^{i \omega_{0} z_{1}}
$$

Eqs. (89,90,92) therefore imply that $\mathbf{I}_{n}$ obeys the recursion relation

$$
\mathbf{I}_{n}=C_{A}\left(e^{i\left(\omega_{0}-\omega_{n}\right) z_{n}} e^{i \mathbf{q} n \cdot \hat{\mathbf{b}}}-1\right) \mathbf{I}_{n-1}-\delta_{n, 1} C_{A} C_{R} \mathbf{B}_{1} e^{i \omega_{0} z_{1}}
$$

With $\mathbf{I}_{0}=-C_{R} \mathbf{H} e^{i \omega_{0} z_{0}}$ and Eq. (93) for $n \geq 1$ we solve (94) in a closed form

$$
\mathbf{I}_{n}=C_{R} C_{A}^{n}\left\{\prod_{m=1}^{n}\left(e^{i\left(\omega_{0}-\omega_{m}\right) z_{m}} e^{i \mathbf{q}_{m} \cdot \hat{\mathbf{b}}}-1\right)\right\} \mathbf{H}\left(e^{i \omega_{0} z_{1}}-e^{i \omega_{0} z_{0}}\right)
$$

where the product is understood as ordered for left to right in decreasing order in operators labeled by $m$.

Combining Eqs. (35,86,87,88,95) that specify how the reaction operator acts between the contraction of rank $n-1$ classes, we find that the inclusive radiation probability, Eq. (63), obeys a simple recursion relation:

$$
P_{n}(\mathbf{k})=C_{A}\left(P_{n-1}\left(\mathbf{k}-\mathbf{q}_{n}\right)-P_{n-1}(\mathbf{k})\right)-2 C_{A} \mathbf{B}_{n} \cdot\left(\boldsymbol{R e} e^{-i \omega_{n} z_{n}} e^{i \mathbf{q}_{\mathbf{n}} \cdot \hat{\mathbf{b}}} \mathbf{I}_{n-1}\right)+\delta_{n, 1} C_{A} C_{R}\left|\mathbf{B}_{1}\right|^{2}
$$

We can solve Eq. (96) with the initial condition

$$
P_{0}=C_{R} \mathbf{H}^{2}
$$

We introduce the following notation for the separation of the scattering centers $\Delta z_{n} \equiv z_{n}-z_{n-1}$. For $n=1$ the solution is

$$
P_{1}=C_{A} C_{R}\left(\mathbf{C}_{1}^{2}-\mathbf{H}^{2}+\mathbf{B}_{1}^{2}+2 \mathbf{B}_{1} \cdot \mathbf{C}_{1} \cos \left(\omega_{1} \Delta z_{1}\right)\right)=-2 C_{A} C_{R} \mathbf{B}_{1} \cdot \mathbf{C}_{1}\left(1-\cos \left(\omega_{1} \Delta z_{1}\right)\right)
$$

For $n=2$ we need

$$
\begin{aligned}
& \operatorname{Re} e^{-i \omega_{2} z_{2}} I_{1}\left(\mathbf{k}-\mathbf{q}_{2}\right)=-C_{A} C_{R} \operatorname{Re} e^{-i \omega_{2} z_{2}}\left(\mathbf{C}_{(12)}\left(e^{i\left(\omega_{2} z_{1}-\omega_{(12)} \Delta z_{1}\right)}-e^{i \omega_{2} z_{1}}\right)-\mathbf{C}_{2}\left(e^{i \omega_{2} z_{0}}-e^{i \omega_{2} z_{1}}\right)\right) \\
&=C_{A} C_{R}\left[-\mathbf{C}_{(12)}\left(\cos \left(\omega_{2} \Delta z_{2}+\omega_{(12)} \Delta z_{1}\right)-\cos \left(\omega_{2} \Delta z_{2}\right)\right)\right. \\
&+\mathbf{C}_{2}\left(\cos \left(\omega_{2}\left(\Delta z_{1}+\Delta z_{2}\right)-\cos \left(\omega_{2} \Delta z_{2}\right)\right)\right]
\end{aligned}
$$

Therefore, the $n=2$ distribution is given by

$$
\begin{gathered}
P_{2}=2 C_{A}^{2} C_{R}\left[\mathbf{B}_{1} \cdot \mathbf{C}_{1}\left(1-\cos \left(\omega_{1} \Delta z_{1}\right)\right)-\mathbf{B}_{2(12)} \cdot \mathbf{C}_{(12)}\left(1-\cos \left(\omega_{(12)} \Delta z_{1}\right)\right)\right. \\
+\mathbf{B}_{2} \cdot \mathbf{C}_{(12)}\left(\cos \left(\omega_{2} \Delta z_{2}+\omega_{(12)} \Delta z_{1}\right)-\cos \left(\omega_{2} \Delta z_{2}\right)\right) \\
-\mathbf{B}_{2} \cdot \mathbf{C}_{2}\left(\cos \left(\omega_{2}\left(\Delta z_{1}+\Delta z_{2}\right)-\cos \left(\omega_{2} \Delta z_{2}\right)\right)\right] .
\end{gathered}
$$

For $n>1$, we can use (95) to obtain the general solution for the gluon probability at $\mathrm{n}^{\text {th }}$ order in opacity

$$
\begin{aligned}
& P_{n}(\mathbf{k})=C_{A}\left(e^{i \mathbf{q}_{n} \cdot \hat{\mathbf{b}}}-1\right) P_{n-1}(\mathbf{k})-2 C_{R} C_{A}^{n} \mathbf{B}_{n} \cdot \mathbf{R e} e^{-i \omega_{n} z_{n}} e^{i \mathbf{q}_{\mathbf{n}} \cdot \hat{\mathbf{b}}}\left\{\prod_{m=1}^{n-1}\left(e^{i\left(\omega_{0}-\omega_{m}\right) z_{m}} e^{i \mathbf{q} m \cdot \hat{\mathbf{b}}}-1\right)\right\} \mathbf{H}\left(e^{i \omega_{0} z_{1}}-e^{i \omega_{0} z_{0}}\right) \\
&=-2 C_{R} C_{A}^{n} \mathbf{R e} \sum_{i=1}^{n}\left\{\prod_{j=i+1}^{n}\left(e^{i \mathbf{q}_{j} \cdot \hat{\mathbf{b}}}-1\right)\right\} \mathbf{B}_{i} \cdot e^{i \mathbf{q}_{i} \cdot \hat{\mathbf{b}}} e^{-i \omega_{0} z_{i}} \times \\
&\left.\qquad \prod_{m=1}^{i-1}\left(e^{i\left(\omega_{0}-\omega_{m}\right) z_{m}} e^{i \mathbf{q}_{m} \cdot \hat{\mathbf{b}}}-1\right)\right\} \mathbf{H}\left(e^{i \omega_{0} z_{1}}-e^{i \omega_{0} z_{0}}\right)
\end{aligned}
$$


where the last applied to $n=1$ as well. This is therefore the complete solution to the problem and is the central result of this paper. Eq. (101) provides not only an algebraic proof of color triviality of the inclusive gluon distribution $\left(P_{n} \propto C_{R} C_{A}^{n}\right)$, but in fact gives the complete angular dependence for arbitrary $z_{i}$ and $\mathbf{q}_{n}$. It also applies to both quark and gluon jets. The derivation through the reaction operator and recursion relations is furthermore much simpler and transparent than previous derivations [11, 13, 34]. The result is also more general and versatile for applications to nuclear collisions.

In this form, it is for example possible to implement $P_{n}$ directly as an "after-burner" in a Monte Carlo event generator, e.g. ZPC [35, 36] or MPC [37, 38]. Such programs solve relativistic 3+1D transport theory and provide much more realistic distributions of target parton coordinates $z_{i}$ along the path of a high energy jet in nuclear collisions. Eq. (101) also makes it possible to consider evolving potentials since the distribution of the $\mathbf{q}_{n}$ remain unspecified. For example, the screening scale $\mu$, that characterizes the mean $\mathbf{q}_{n}$ transfered at $z_{n}$, could itself depend on $n$ because of the variation of the local density at that point. In this way, jet quenching in realistic, evolving nuclear geometries can be calculated more accurately.

\section{Ensemble average over momentum transfers}

We now consider the integrated gluon distribution over the $\mathbf{q}_{m}$ via the normalized squared scattering potential Eq. (53)

$$
\left\langle P_{n}(\mathbf{k})\right\rangle_{v}=\int \prod_{m=1}^{n}\left\{d \mathbf{q}_{m} \bar{v}_{m}^{2}\left(\mathbf{q}_{m}\right)\right\} P_{n}\left(\mathbf{k} ; \mathbf{q}_{1} \cdots \mathbf{q}_{n}\right)
$$

where we allow for the possibility that the effective potential could change along the path of the jet. We hold the $z_{m}$ fixed as yet. ¿From Eq. (96) we need to consider for example

$$
\int d \mathbf{q}_{n} \bar{v}_{n}^{2}\left(\mathbf{q}_{n}\right)\left(P_{n-1}\left(\mathbf{k}-\mathbf{q}_{n}\right)-P_{n-1}(\mathbf{k})\right)=\int d \mathbf{q}_{n}\left(\bar{v}_{n}^{2}\left(\mathbf{q}_{n}\right)-\delta^{2}\left(\mathbf{q}_{n}\right)\right) P_{n-1}\left(\mathbf{k}-\mathbf{q}_{n}\right) .
$$

Since $\delta^{2}\left(\mathbf{q}_{n}\right) \mathbf{B}_{n} \equiv 0$, we can also replace $\bar{v}_{n}^{2}\left(\mathbf{q}_{n}\right)$ by $\bar{v}_{n}^{2}\left(\mathbf{q}_{n}\right)-\delta^{2}\left(\mathbf{q}_{n}\right)$ in the average over the inhomogeneous term. However, due to the special form of the operators defining $\mathbf{I}_{n}$ in Eq. (95) we can also rewrite the integral over $\mathbf{q}_{i}$ for $i=1, \cdots, n-1$ in analogous form

$$
\begin{aligned}
\int \prod_{j=1}^{n}\left\{d \mathbf{q}_{j} \bar{v}_{j}^{2}\left(\mathbf{q}_{j}\right)\right\} \mathbf{B}_{n} e^{-i \omega_{n} z_{n}} e^{i \mathbf{q}_{\mathbf{n}} \cdot \hat{\mathbf{b}}} \prod_{m=1}^{n-1}\left(e^{i\left(\omega_{0}-\omega_{m}\right) z_{m}} e^{i \mathbf{q} m \cdot \hat{\mathbf{b}}}-1\right) \\
\quad=\int \prod_{j=1}^{n}\left\{d \mathbf{q}_{j}\left[\bar{v}_{j}^{2}\left(\mathbf{q}_{j}\right)-\delta^{2}\left(\mathbf{q}_{j}\right)\right]\right\} \mathbf{B}_{n} e^{-i \omega_{0} z_{n}} \prod_{m=1}^{n} e^{i\left(\omega_{0}-\omega_{m}\right) z_{m}} e^{i \mathbf{q}_{\mathbf{m}} \cdot \hat{\mathbf{b}}},
\end{aligned}
$$

where the product of operators is again path ordered. This product of operators can be simplified as follows

$$
\begin{aligned}
e^{-i \omega_{0} z_{n}} \prod_{j=1}^{n} e^{i\left(\omega_{0}-\omega_{j}\right) z_{j}} e^{i \mathbf{q}_{\mathbf{j}} \cdot \hat{\mathbf{b}}} & =e^{-i \omega_{n} z_{n}} e^{i\left(\omega_{n}-\omega_{(n-1, n)}\right) z_{n-1}} \cdots e^{i\left(\omega_{(2 \cdots n)}-\omega_{(1 \cdots n)}\right) z_{1}} e^{i \mathbf{Q} \cdot \hat{\mathbf{b}}} \\
& =\exp \left(-i \sum_{j=1}^{n} \omega_{(j \cdots n)}\left(z_{j}-z_{j-1}\right)\right) e^{-i \omega_{(1 \cdots n)} z_{0}} e^{i \mathbf{Q} \cdot \hat{\mathbf{b}}}
\end{aligned}
$$

where $\mathbf{Q}=\sum \mathbf{q}_{m}$ is the total momentum transfer, and

$$
\omega_{(m, \cdots, n)}=\frac{\left(\mathbf{k}-\mathbf{q}_{m}-\cdots-\mathbf{q}_{n}\right)^{2}}{2 x E} .
$$

Therefore, we can evaluate

$$
e^{-i \omega_{0} z_{n}} \prod_{j=1}^{n} e^{i\left(\omega_{0}-\omega_{j}\right) z_{j}} e^{i \mathbf{q}_{\mathbf{j}} \cdot \hat{\mathbf{b}}} \mathbf{H}\left(e^{i \omega_{0} z_{1}}-e^{i \omega_{0} z_{0}}\right)=\mathbf{C}_{(1 \cdots n)} e^{i \Phi_{n, n}}\left(e^{i \omega_{(1 \cdots n)}\left(z_{1}-z_{0}\right)}-1\right)
$$


where $\Phi_{n, n}$ is the gluon elastic scattering phase shift from $z_{0}$ to $z_{n}$. The partial (eikonal) phase shift $\left(\int_{z_{m-1}}^{z_{n}} d z\left(k_{z}(z)-x E\right)\right)$ due to gluon rescattering from $z_{m-1}$ to $z_{n}$ is given by

$$
\Phi_{n, m}=-\sum_{k=1}^{m} \omega_{(k \cdots n)}\left(z_{k}-z_{k-1}\right)=-\sum_{k=1}^{m} \omega_{(k \cdots n)} \Delta z_{k} .
$$

Eq. (107) makes it possible to write the recursion relation for the momentum transfer averaged probability for $n>1$ in the form

$$
\begin{aligned}
\left\langle P_{n}\right\rangle_{v}= & C_{A} \int d \mathbf{q}_{n}\left(\bar{v}_{n}^{2}\left(\mathbf{q}_{n}\right)-\delta^{2}\left(\mathbf{q}_{n}\right)\right)\left\langle P_{n-1}\left(\mathbf{k}-\mathbf{q}_{n}\right)\right\rangle_{v} \\
& -2 C_{R} C_{A}^{n} \int \prod_{i=1}^{n}\left\{d \mathbf{q}_{i}\left(\bar{v}_{i}^{2}\left(\mathbf{q}_{i}\right)-\delta^{2}\left(\mathbf{q}_{i}\right)\right)\right\} \mathbf{B}_{n} \cdot \mathbf{C}_{(1 \cdots n)} \operatorname{Re} e^{i \Phi_{n, n}}\left(e^{i \omega_{(1 \cdots n)}\left(z_{1}-z_{0}\right)}-1\right) .
\end{aligned}
$$

With $P_{1}$ given by Eq. (98), the solution for $n>0$ is

$$
\begin{aligned}
\left\langle P_{n}\right\rangle_{v}=-2 C_{R} C_{A}^{n} \int \prod_{i=1}^{n}\{ & \left.d \mathbf{q}_{i}\left(\bar{v}_{i}^{2}\left(\mathbf{q}_{i}\right)-\delta^{2}\left(\mathbf{q}_{i}\right)\right)\right\} \\
& \times \sum_{m=1}^{n} \mathbf{B}_{(m+1, \cdots, n)(m \cdots n)} \cdot \mathbf{C}_{(1 \cdots n)} \mathbf{R e}\left(e^{i \Phi_{n, m}}\left(e^{i \omega_{(1 \cdots n)}\left(z_{1}-z_{0}\right)}-1\right)\right) .
\end{aligned}
$$

where for $m=n, \mathbf{B}_{(n+1 \cdots n)(n)} \equiv \mathbf{B}_{(0)(n)} \equiv \mathbf{B}_{(n)}$.

Recall that we cannot take the contact limit $z_{1}=z_{0}$, which apparently vanishes, because our derivation assumed that $z_{m}-z_{m-1}$ were larger than the range of the force $1 / \mu$. However, it is clear from Eq. (110), that it is the first step phase difference

$$
e^{i \omega_{(1 \cdots n)}\left(z_{1}-z_{0}\right)}-1
$$

that controls the bulk of the LPM destructive interference effect that suppresses radiation when the formation length $1 / \omega_{(1 \cdots n)}$ is long compared to $\left(z_{1}-z_{0}\right)$. The subsequent interactions merely modulate this effect with an elastic scattering phase shift.

Finally, we can restore the proportionality constants by multiplying with $\alpha_{s} / \pi$ for the production vertex, $1 / \pi$ for the $d^{2} \mathbf{k}$ measure, and $\prod_{j}\left(\sigma_{g}(j) / A_{\perp}\right)$ along the path to convert the $\bar{v}_{j}$ back into $v_{j}$ from (53). In addition, we must multiply by the combinatorial factor,

$$
\frac{N !}{n !(N-n) !} \approx \frac{N^{n}}{n !}
$$

that counts the number of ways $n$ target partons out of $N$ can be within the interaction range of the jet+gluon system. Including these factors, the general formula for the induced gluon number distribution can finally be written as

$$
\begin{aligned}
x \frac{d N^{(n)}}{d x d^{2} \mathbf{k}}= & \frac{C_{R} \alpha_{s}}{\pi^{2}} \frac{1}{n !}\left(\frac{L}{\lambda_{g}(1)}\right)^{n} \int \prod_{i=1}^{n}\left\{d \mathbf{q}_{i}\left(\frac{\lambda_{g}(1)}{\lambda_{g}(i)}\right)\left(\bar{v}_{i}^{2}\left(\mathbf{q}_{i}\right)-\delta^{2}\left(\mathbf{q}_{i}\right)\right)\right\} \\
& \times\left[-2 \mathbf{C}_{(1, \cdots, n)} \cdot \sum_{m=1}^{n} \mathbf{B}_{(m+1, \cdots, n)(m, \cdots, n)}\left(\cos \left(\sum_{k=2}^{m} \omega_{(k, \cdots, n)} \Delta z_{k}\right)-\cos \left(\sum_{k=1}^{m} \omega_{(k, \cdots, n)} \Delta z_{k}\right)\right)\right]
\end{aligned}
$$

where $\sum_{2}^{1} \equiv 0$ is understood.

We emphasize that Eq. (113) is not restricted to uncorrelated geometries as in [ 11, 34. Also it allows the inclusion of finite kinematic boundaries on the $\mathbf{q}_{i}$ as well as different functional forms and elastic cross sections $\sigma_{g}(i)$ along the eikonal path. 
For particular models of the target geometry one can proceed further analytically. For a sharp rectangular geometry, the average over the longitudinal target profile with

$$
\bar{\rho}\left(z_{1}, \cdots, z_{n}\right)=\frac{n !}{L^{n}} \theta\left(L-z_{n}\right) \theta\left(z_{n}-z_{n-1}\right) \cdots \theta\left(z_{2}-z_{1}\right)
$$

leads to an oscillatory pattern [34] that is an artifact of the assumed sharp edges.

A somewhat more realistic model [27, 28] utilizes normalized exponential longitudinal distributions of scattering center separations:

$$
\bar{\rho}\left(z_{1}, \cdots, z_{n}\right)=\prod_{j=1}^{n} \frac{\theta\left(\Delta z_{j}\right)}{L_{e}(n)} e^{-\Delta z_{j} / L_{e}(n)},
$$

This converts the oscillating formation physics factors in Eq. 113) into simple Lorentzian factors

$$
\int d \bar{\rho} \cos \left(\sum_{k=j}^{m} \omega_{(k, \cdots, n)} \Delta z_{k}\right)=\operatorname{Re} \prod_{k=j}^{m} \frac{1}{1+i \omega_{(k, \cdots, n)} L_{e}(n)}
$$

In order to fix $L_{e}(n)$, we require that $\left\langle z_{k}-z_{0}\right\rangle=k L /(n+1)$ for both geometries (114, 115). This constrains $L_{e}(n)=L /(n+1)[27,28]$.

Note finally that the ratio of the medium induced to the medium independent factorization gluon distributions vanish at both small and large $|\mathbf{k}|$

$$
\lim _{|\mathbf{k}|=0 \text { and } \infty} \frac{d N^{(n)}}{d N^{(0)}}=0
$$

As $|\mathbf{k}| \rightarrow 0, d N^{(0)} \rightarrow \infty$ while all but one term in $d N^{(n)}$ is finite. The potentially singular term $\propto$ $\left(1 / \mathbf{k}^{2}\right) \int \sum_{j} \mathbf{k} \cdot \mathbf{q}_{j}$ however vanishes due to azimuthal integrations. In the $|\mathbf{k}| \rightarrow \infty$ limit, on the other hand, all Gunion-Bertsch currents $\mathbf{B}_{(. .),(.)} \rightarrow O\left(1 / \mathbf{k}^{4}\right)$ and hence vanish faster than $1 / \mathbf{k}^{2}$ from $d N^{(0)}$. This observation partly justifies neglecting the kinematical $|\mathbf{k}|$ boundaries (120) in analytical approximations.

\section{NUMERICAL ESTIMATES}

\section{A. Angular distributions}

Figs. 4a-c illustrate Eq. (113) for a exponential geometry (115) equivalent to a box of thickness $L=5$ $\mathrm{fm}$ with $\lambda_{g}=1 \mathrm{fm}$ including kinematical constraints appropriate for a $50 \mathrm{GeV}$ jet. We used adaptive Monte-Carlo integration [39, 40] to integrate over the momentum transfers $\mathbf{q}_{1} \cdots \mathbf{q}_{n}$.

The medium induced gluon differential distributions up to third order in opacity are plotted divided by the zeroth order in opacity, hard distribution Eq. (119) for radiated gluons with light-cone momentum fractions $x=0.05,0.2,0.5$. Note that $d N^{(i n d)} / d N^{(0)}$ tends to vanish at both small and large $k$ in accordance with Eq. (117). As $x$ increases the relative magnitude of the medium induced contribution decrease and the overall magnitude is set already by the first order in opacity result. Higher orders redistribute the moderate $k^{2} / \mu^{2} \sim 10$ toward higher values due to elastic rescattering of the gluon, but they also tend to fill in the low $k$ region with additional soft radiation. The overall angular pattern appears somewhat complex, but one must keep in mind that the variation is rather slow since the logarithmic scale varies over several orders of magnitude.

Fig. $4 \mathrm{~d}$ shows the actual rather modest size of the induced radiation contribution on top of the hard $1 / \mathbf{k}^{2}$ distribution for the case of $x=0.05$.

\section{B. Intensity distribution and energy loss to first order in opacity. Analytical approach}




\section{Zero Opacity Limit}

The jet distribution in the absence of final state interactions is given by [27]

$$
d^{3} N_{J}=\rho^{(0)}(\overrightarrow{\mathbf{p}}) \frac{d^{3} \overrightarrow{\mathbf{p}}}{2|\overrightarrow{\mathbf{p}}|(2 \pi)^{3}}=d_{R}|J(|\overrightarrow{\mathbf{p}}|, \overrightarrow{\mathbf{p}})|^{2} \frac{d^{3} \overrightarrow{\mathbf{p}}}{2|\overrightarrow{\mathbf{p}}|(2 \pi)^{3}},
$$

In the Leading Pole Approximation (LPA) approximation [33], the radiation distribution accompanying the such hard processes for a spin $\frac{1}{2}$ jet is given by

$$
x \frac{d N^{(0)}}{d x d \mathbf{k}^{2}} \approx \frac{C_{R} \alpha_{s}}{\pi}\left(1-x+\frac{x^{2}}{2}\right) \frac{1}{\mathbf{k}^{2}},
$$

where in the eikonal approximation the light-cone momentum fraction $x=k^{+} / E^{+} \approx \omega / E$ and $\mathbf{k}$ is assumed to be small compared to $\omega$. For other spin jets, another suitable splitting function replaces the $x$ dependence above.
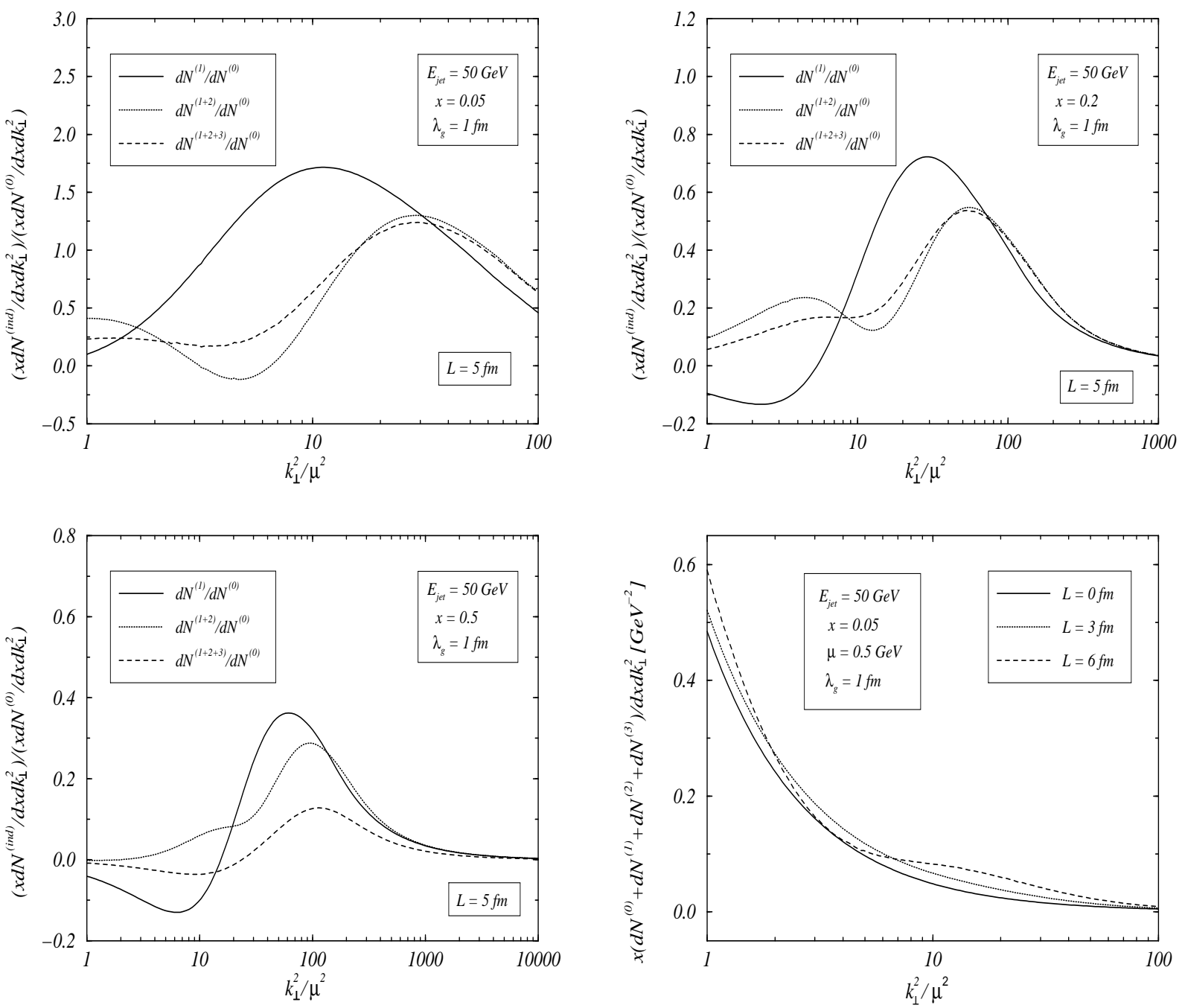
FIG 4. (a)-(c) The medium-induced double differential gluon distributions are plotted vs. $\mathbf{k}^{2} / \mu^{2}$ up to first $\left(d N^{(1)}\right)$, second $\left(d N^{(1+2)}\right)$ and third $\left(d N^{(1+2+3)}\right)$ order in the opacity $\left(L / \lambda_{g}\right)$ expansion, divided by the medium independent hard radiation $\left(d N^{(0)}\right) \propto 1 / k^{2}$. Curves are obtained numerically for exponential geometry with $L / \lambda_{g}=5, E_{j e t}=50 \mathrm{GeV}$ and $\mu=0.5 \mathrm{GeV}$. The first three figures (a)-(c) are for typical soft, semi-soft and hard gluons respectively, $x=0.05,0.2,0.5$. (d) The full gluon differential distribution up to third order in opacity $d N^{(0)}+d N^{(1)}+d N^{(2)}+d N^{(3)}$ is shown for $x=0.05$ for $L=0,3,6 \mathrm{fm}$.

We consider radiation outside a cone with $|\mathbf{k}|>\mu$. and with the upper $|\mathbf{k}|$ bound determined from the three body (jet+jet+glue) kinematics. The gluon kinematic boundaries are therefore

$$
\mathbf{k}_{\min }^{2}=\mu^{2}, \quad \mathbf{k}_{\max }^{2}=\min \left[4 E^{2} x^{2}, 4 E^{2} x(1-x)\right] .
$$

The radiation intensity integrated over this range of $\mathbf{k}$ is

$$
\frac{d I^{(0)}}{d x}=\frac{2 C_{R} \alpha_{s}}{\pi}\left(1-x+\frac{x^{2}}{2}\right) E \log \frac{|\mathbf{k}|_{\max }}{|\mathbf{k}|_{\min }},
$$

where $|\mathbf{k}|_{\max }$ and $|\mathbf{k}|_{\min }$ are given by Eq. (120). Note that the differential intensity is roughly uniform with the exception of the kinematical edges $x \rightarrow 0$ and $x \rightarrow 1$ as seen in Fig. 5a. In the Leading Log of the Leading Pole Approximation (LLA) the radiative total energy loss of a quark jet originating from a hard vertex outside a cone defined by Eq. (120) is given by

$$
\Delta E^{(0)}=\frac{4 C_{R} \alpha_{s}}{3 \pi} E \log \frac{E}{\mu} .
$$

While this overestimates the radiative energy loss in the vacuum (self-quenching), it is important to note that $\Delta E^{(0)} / E \sim 50 \%$ is typically rather large. As shown below, the medium induced energy loss is small by comparison. For a gluon jet one has to replace the quark splitting function $q \rightarrow q g$ by the gluon one $g \rightarrow g g$ in Eq. (121) and use $C_{A} \equiv N_{c} \approx 2 C_{F}$.

The total energy loss should reduce to the medium independent one in the limit of vanishing opacity

$$
\lim _{L \rightarrow 0} \frac{\Delta E^{(t o t)}}{\Delta E^{(0)}}=1
$$

and for jets of asymptotically high energies due to factorization

$$
\lim _{E \rightarrow \infty} \frac{\Delta E^{(t o t)}}{\Delta E^{(0)}}=1
$$

\section{First Order in Opacity Correction}

The first order $(n=1)$ in opacity contribution, $d I^{(1)} / d x$, to the induced radiation intensity can be read of from Eq. (113). The longitudinal coordinate average over the equivalent exponential target profile (115) is done with $L_{e}(2)=L / 2$. Including the $q \rightarrow q g$ splitting function as in (119) we have

$$
\begin{aligned}
\frac{d I^{(1)}}{d x}=\frac{C_{R} \alpha_{s}}{\pi}\left(1-x+\frac{x^{2}}{2}\right) \frac{L}{\lambda_{g}} E \int_{\mathbf{k}_{\min }^{2}}^{\mathbf{k}_{\max }^{2}} \frac{d \mathbf{k}^{2}}{\mathbf{k}^{2}} & \\
& \int_{0}^{\mathbf{q}_{1 \max }^{2}} d^{2} \mathbf{q}_{1} \frac{\mu_{e f f}^{2}}{\pi\left(\mathbf{q}_{1}^{2}+\mu^{2}\right)^{2}} \frac{2 \mathbf{k} \cdot \mathbf{q}_{1}\left(\mathbf{k}-\mathbf{q}_{1}\right)^{2} L^{2}}{16 x^{2} E^{2}+\left(\mathbf{k}-\mathbf{q}_{1}\right)^{4} L^{2}} .
\end{aligned}
$$

To obtain a simple analytic result, we ignore the kinematic boundaries and set $|\mathbf{k}|_{\min }=0,|\mathbf{k}|_{\max }=\infty$ that is motivated by Eq. (117)). We also set $\left|\mathbf{q}_{1}\right|_{\max }=\infty$ (i.e. $\left.\mu_{\text {eff }}^{2}=\mu^{2}\right)$. This allows us to change variables $\mathbf{q}^{\prime} \equiv \mathbf{k}-\mathbf{q}_{1}$ in Eq. 125 ) 


$$
\begin{aligned}
\frac{d I^{(1)}}{d x}=\frac{C_{R} \alpha_{s}}{\pi}\left(1-x+\frac{x^{2}}{2}\right) & \frac{\mu^{2} L}{\lambda_{g}} E \int_{0}^{\infty} d \mathbf{q}^{\prime 2} \frac{\mathbf{q}^{\prime 2} L^{2}}{16 x^{2} E^{2}+\mathbf{q}^{\prime 4} L^{2}} \\
& \int_{0}^{\infty} \frac{d \mathbf{k}^{2}}{\mathbf{k}^{2}} \int_{0}^{2 \pi} \frac{d \phi}{2 \pi} \frac{2 \mathbf{k} \cdot\left(\mathbf{k}+\mathbf{q}^{\prime}\right)}{\left(\mathbf{q}^{\prime 2}+\mathbf{k}^{2}+2\left|\mathbf{q}^{\prime}\right||\mathbf{k}| \cos (\phi)+\mu^{2}\right)^{2}}
\end{aligned}
$$

and express the integrand in the azimuthal $\phi$ integral as a partial derivative with respect to $\mathbf{k}^{2}$

$$
-2 \mathbf{k}^{2} \partial_{\mathbf{k}^{2}} \int_{0}^{2 \pi} \frac{d \phi}{2 \pi} \frac{1}{\left(\mathbf{q}^{\prime 2}+\mathbf{k}^{2}+2\left|\mathbf{q}^{\prime}\right||\mathbf{k}| \cos (\phi)+\mu^{2}\right)}=-2 \mathbf{k}^{2} \partial_{\mathbf{k}^{2}} \frac{1}{\sqrt{\left(\left(\mathbf{k}^{2}+\mathbf{q}^{\prime 2}+\mu^{2}\right)^{2}-4 \mathbf{k}^{2} \mathbf{q}^{\prime 2}\right)}} .
$$

The remaining $\mathbf{q}^{\prime}$ integral

$$
\frac{d I^{(1)}}{d x}=\frac{C_{R} \alpha_{s}}{\pi}\left(1-x+\frac{x^{2}}{2}\right) \frac{L}{\lambda_{g}} E \int_{0}^{\infty} d \mathbf{q}^{\prime 2} \frac{2 \mu^{2}}{\mathbf{q}^{\prime 2}+\mu^{2}} \frac{\mathbf{q}^{\prime 2} L^{2}}{16 x^{2} E^{2}+\mathbf{q}^{\prime 4} L^{2}} .
$$

can be performed then analytically, resulting in

$$
\frac{d I^{(1)}}{d x}=\frac{C_{R} \alpha_{s}}{\pi}\left(1-x+\frac{x^{2}}{2}\right) E \frac{L}{\lambda_{g}} f(\gamma), \quad \gamma=\frac{L \mu^{2}}{4 x E},
$$

where $\gamma$ is a measure of the formation probability. The formation function $f(\gamma)$ is given by

$$
f(\gamma)=\frac{\gamma(\pi+2 \gamma \log \gamma)}{\left(1+\gamma^{2}\right)} \approx \begin{cases}\pi \gamma & \text { if } \quad \gamma \ll 1 \\ 2 \log \gamma & \text { if } \quad \gamma \gg 1\end{cases}
$$

It is the $\gamma \ll 1$ limit of the formation function Eq. (129) in which the the first order in opacity mediuminduced intensity distribution reduces to a simple form with a characteristic quadratic dependence on $L$ :

$$
\frac{d I^{(1)}}{d x} \approx \frac{C_{R} \alpha_{s}}{4} \frac{1-x+\frac{x^{2}}{2}}{x} \frac{L^{2} \mu^{2}}{\lambda_{g}}
$$

This formula breaks down at both $x \rightarrow 0$ and $x \rightarrow 1$ because $|\mathbf{k}|_{\max }$ and $\left|\mathbf{q}_{\mathbf{1}}\right|_{\max }$ cannot be approximated by $\infty$ and because the small $x$ approximations used above break down as $x \rightarrow 1$.

The induced radiative energy loss to first order in opacity in the framework of the above approximations is then given by

$$
\Delta E^{(1)}=\frac{C_{R} \alpha_{s}}{4} \frac{L^{2} \mu^{2}}{\lambda_{g}} \log \frac{E}{\mu} .
$$

This equation is directly comparable to Eq.(??) of Ref. [12].

\section{Induced radiation intensity to higher orders in opacity. Numerical results}

Whereas Eq. (131) displays analytically the main qualitative features of non-abelian energy loss, in practice at the finite energies available kinematical bounds do affect quantitatively the results. This naturally leads to a reduction relative to the analytic estimate.

In Fig. 5a the induced intensity distribution $d I^{(i n d)} / d x$ (sets of dashed curves) is compared to the medium independent $d I^{(0)}$. We consider the example of a $50 \mathrm{GeV}$ quark jet $(\mu=0.5 \mathrm{GeV})$ at RHIC. The hard radiation intensity result is roughly constant with $x$, whereas for most of the $x$ region the induced radiation intensity falls like $1 / x^{\alpha}, \alpha \sim 1$. We note that for relatively thin plasmas $L / \lambda_{g} \leq 3$ the medium induced energy loss remains small compared to $d I^{(0)}$. 

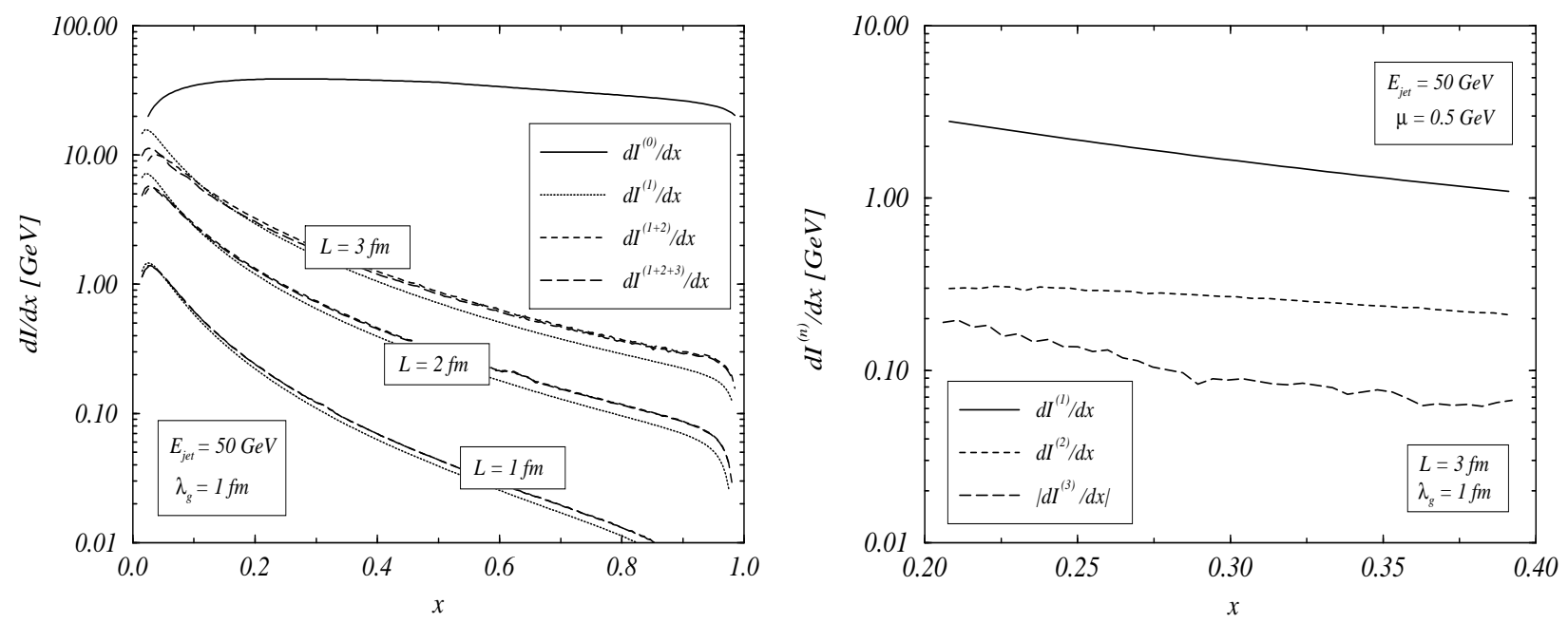

FIG 5. (a) The radiation intensity distribution is plotted vs. the light-cone momentum fraction $x$ of the gluon. We consider a $50 \mathrm{GeV}$ quark jet in a plasma with screening scale $\mu=0.5 \mathrm{GeV}$ and $\lambda_{g}=1$ $\mathrm{fm}$. The solid curve shows the dominant medium-independent radiation intensity. The medium-induced gluon spectrum is plotted for up to third order in opacity $\left(d I^{(1)}, d I^{(1+2)}\right.$ and $\left.d I^{(1+2+3)}\right)$ for opacities $L / \lambda_{g}=1,2,3$. (b) The absolute value of the orders in opacity $d I^{(1)}, d I^{(2)}$ and $d I^{(3)}$ that contribute in part (a) are plotted for the same energy and opacity $L / \lambda_{g}=3$.

It is surprising how much the first order result dominates the induced intensity distribution[28]. Fig. 5b illustrates how rapidly the contributions from higher orders in opacity decrease. While the differential angular distribution continue to show some weak sensitivity to higher order contributions in opacity, the angle integrated intensity is much less affected beyond first order. The third order contribution is almost buried in the "numerical noise". For opacities $L / \lambda_{g} \simeq 6$ some more pronounced probability redistribution can be seen in the double differential level Fig. 4. However, when integrated over $\mathbf{k}^{2}$ and then over $x_{\min }<x<x_{\max }$ to obtain the induced non-abelian energy loss $\Delta E$ (ind), the first order result dominates very strongly as seen in Fig. 6. 


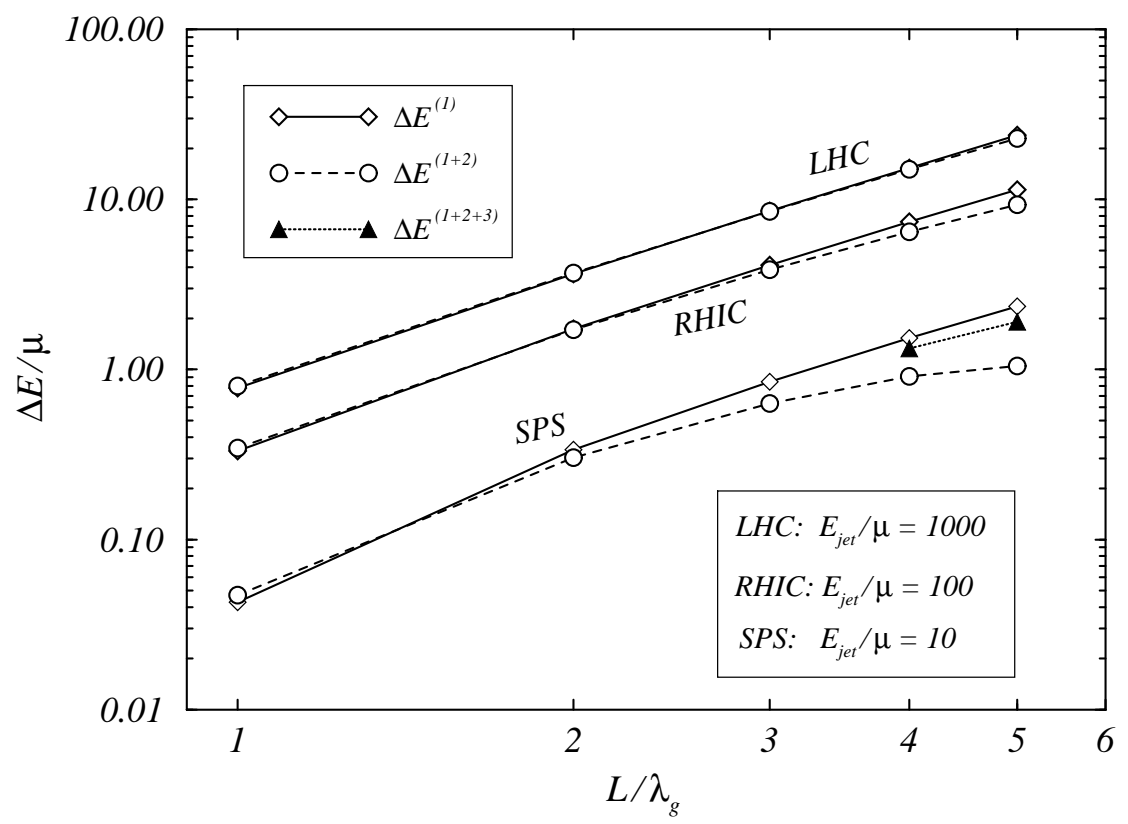

FIG 6. The radiated energy loss of a quark jet with energy $E_{\text {jet }}=5,50,500 \mathrm{GeV}$ (at SPS, RHIC, LHC) is plotted as a function of the opacity $L / \lambda_{g} .\left(\lambda_{g}=1 \mathrm{fm}, \mu=0.5 \mathrm{GeV}\right)$. Solid curves show the first order in opacity results. The dashed curves show results up to second order in opacity, and two third order results are shown by solid triangles for SPS energies.

The induced energy loss shown in Fig. 6 is for quark jets with energies $E_{j e t}=5,50,500 \mathrm{GeV}$ typical for SPS, RHIC and LHC. Higher orders in opacity $\left(L / \lambda_{g}\right)^{n}, \quad n \geq 2$ have little effect except at very low SPS energies. The kinematic bounds at SPS suppress very much the energy loss in comparison to RHIC and LHC energies. An analysis of the slopes as a function of the opacity, $L / \lambda_{g}$, shows that $\Delta E($ ind $) \propto L^{2 \pm 0.1}$ at all energies even with finite kinematic boundaries included. As a measure of the deviation of the simple first order analytic estimate, we generalize Eq. (131) as follows:

$$
\Delta E^{(i n d)}=\frac{C_{R} \alpha_{s}}{N(E)} \frac{L^{2} \mu^{2}}{\lambda_{g}} \log \frac{E}{\mu},
$$

If the kinematic bounds are ignored as in Eq. (130), then $N(E)=4$. Including finite kinematic constraints cause $N(E)$ to deviate considerably from this asymptotic value. We find numerically that $N(E)=7.3,10.1,24.4$ for $E=500,50,5 \mathrm{GeV}$. Together with the logarithmic dependence of energy, these kinematic effects suppress greatly the energy loss at lower (SPS) energies as seen in Fig. 6 . This is in contrast to the asymptotic, energy independent result quoted in Ref. [11] where the finite kinematic bounds were neglected. Our numerical results, however, agree with Ref. [11] near LHC energies as reported in [28].

\section{Acknowledgments}

We thank Al Mueller and Urs Wiedemann for numerous clarifying discussions. This work was supported by the DOE Research Grant under Contract No. De-FG-02-93ER-40764, partly by the US-Hungarian Joint Fund No.652 and OTKA No. T032796. 
APPENDIX A: DIAGRAMS $M_{1,0,0}=G_{0} X_{1,0}, M_{1,1,0}=G_{0} G^{-1} X_{1,0} G_{1}$ AND $M_{1,0,1}=G_{0} X_{1,1}$

To make contact with the results in Ref. [27, 26] we present explicit calculation of the first nontrivial diagrams shown in Fig. 7.
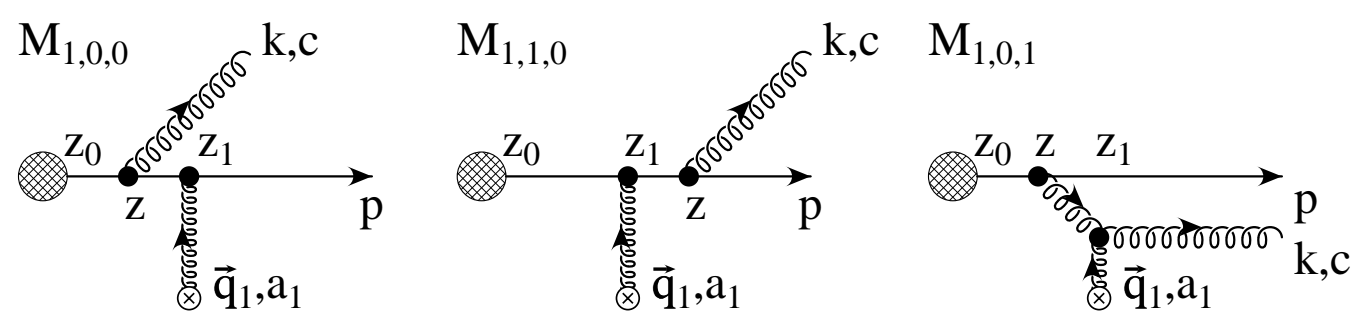

FIG 7. Three "direct" terms $M_{1,0,0}=G_{0} X_{1,0}, M_{1,1,0}=G_{0} G^{-1} X_{1,0} G_{1}$ and $M_{1,0,1}=G_{0} X_{1,1}$ contribute to the soft gluon radiation amplitude to first order in opacity $L / \lambda \propto \sigma_{e l} / A_{\perp}$.

As a first application, consider the one rescattering amplitude $M_{1,0,1}$, in the notation of Ref. [27].

$$
\begin{aligned}
M_{1,0,1}= & \int \frac{d^{4} q_{1}}{(2 \pi)^{4}} i J\left(p+k-q_{1}\right) e^{i\left(p+k-q_{1}\right) x_{0}} \Lambda_{1}\left(p, k, q_{1}\right) V\left(q_{1}\right) e^{i q_{1} x_{1}} \times \\
& \times i \Delta\left(p+k-q_{1}\right)(-i) \Delta\left(k-q_{1}\right) \\
\approx & J(p+k) e^{i(p+k) x_{0}}\left[c, a_{1}\right] T_{a_{1}}(-i) \int \frac{d^{2} \mathbf{q}_{1}}{(2 \pi)^{2}} e^{-i \mathbf{q}_{1} \cdot\left(\mathbf{x}_{1}-\mathbf{x}_{0}\right)} 2 g_{s} \boldsymbol{\epsilon} \cdot\left(\mathbf{k}-\mathbf{q}_{1}\right) \times \\
& \times E^{+} \int \frac{d q_{1 z}}{2 \pi} v\left(q_{1 z}, \mathbf{q}_{1}\right) \Delta\left(p+k-q_{1}\right) \Delta\left(k-q_{1}\right) e^{-i q_{1 z}\left(z_{1}-z_{0}\right)} .
\end{aligned}
$$

The longitudinal momentum transfer integral

$$
I_{1}\left(p, k, \mathbf{q}_{1}, z_{1}-z_{0}\right) \equiv \int \frac{d q_{1 z}}{2 \pi} v\left(q_{1 z}, \mathbf{q}_{1}\right) \Delta\left(p+k-q_{1}\right) \Delta\left(k-q_{1}\right) e^{-i q_{1 z}\left(z_{1}-z_{0}\right)}
$$

can be performed via closing the contour below the real axis since $z_{1}>z_{0}$. In addition to the potential singularities at $\pm i \mu_{1}\left(\mu_{i}^{2} \equiv \mu_{i \perp}^{2}=\mathbf{q}_{i}^{2}+\mu^{2}\right)$, the two propagators have four poles in the $q_{1 z}$ plane, which up to leading correction are located at

$$
\begin{array}{ll}
\bar{q}_{1}=E^{+}+i \epsilon, & \bar{q}_{2}=-\omega_{0}-i \epsilon \\
\bar{q}_{3}=k^{+}-\omega_{1}+i \epsilon, & \bar{q}_{4}=-\omega_{0}+\omega_{1}-i \epsilon .
\end{array}
$$

The residues give

$$
\operatorname{Res}\left(\bar{q}_{2}\right) \approx-v\left(-\omega_{0}, \mathbf{q}_{1}\right) \frac{e^{i \omega_{0}\left(z_{1}-z_{0}\right)}}{E^{+} k^{+} \omega_{1}}, \quad \operatorname{Res}\left(\bar{q}_{4}\right) \approx v\left(\omega_{1}-\omega_{0}, \mathbf{q}_{1}\right) \frac{e^{i\left(\omega_{0}-\omega_{1}\right)\left(z_{1}-z_{0}\right)}}{E^{+} k^{+} \omega_{1}}
$$

while

$$
\operatorname{Res}\left(-i \mu_{1}\right) \approx \frac{4 \pi \alpha_{s} e^{-\mu_{1}\left(z_{1}-z_{0}\right)}}{\left(-2 i \mu_{1}\right) E^{+} k^{+}\left(-i \mu_{1}\right)^{2}}
$$

where we assumed that $k^{+} \gg \mu_{1} \gg \omega_{i}$. In the well-separated case where $\mu\left(z_{1}-z_{0}\right)=\mu \lambda \gg 1$, this potential residue is exponentially suppressed and therefore

$$
\begin{aligned}
I_{1}\left(p, k, \mathbf{q}_{1}, z_{1}-z_{0}\right) & \approx i \frac{e^{i \omega_{0}\left(z_{1}-z_{0}\right)}}{E^{+} k^{+} \omega_{1}}\left(v\left(-\omega_{0}, \mathbf{q}_{1}\right)-v\left(\omega_{1}-\omega_{0}, \mathbf{q}_{1}\right) e^{-i \omega_{1}\left(z_{1}-z_{0}\right)}\right) \\
& \approx i v\left(0, \mathbf{q}_{1}\right) \frac{e^{i \omega_{0}\left(z_{1}-z_{0}\right)}}{E^{+} k^{+} \omega_{1}}\left(1-e^{-i \omega_{1}\left(z_{1}-z_{0}\right)}\right) .
\end{aligned}
$$


We thus recover in this eikonal limit the time ordered perturbation result in [27, 26]

$$
\begin{aligned}
M_{1,0,1}= & J(p) e^{i p x_{0}}(-i) \int \frac{d^{2} \mathbf{q}_{1}}{(2 \pi)^{2}} v\left(0, \mathbf{q}_{1}\right) e^{-i \mathbf{q}_{1} \cdot \mathbf{b}_{1}} \times \\
& \times 2 i g_{s} \frac{\boldsymbol{\epsilon} \cdot\left(\mathbf{k}-\mathbf{q}_{1}\right)}{\left(\mathbf{k}-\mathbf{q}_{1}\right)^{2}} e^{i\left(\omega_{0}-\omega_{1}\right) z_{1}}\left(e^{i \omega_{1} z_{1}}-e^{i \omega_{1} z_{0}}\right)\left[c, a_{1}\right] T_{a_{1}},
\end{aligned}
$$

where $\mathbf{b}_{1}=\mathbf{x}_{1}-\mathbf{x}_{0}$. Similarly, we recover the other two amplitudes for one center, e.g.,

$$
\begin{aligned}
M_{1,1,0}= & J(p) e^{i p x_{0}}(-i) \int \frac{d^{2} \mathbf{q}_{1}}{(2 \pi)^{2}} v\left(0, \mathbf{q}_{1}\right) e^{-i \mathbf{q}_{1} \cdot \mathbf{b}_{1}} \times \\
& \times\left(-2 i g_{s}\right) \frac{\boldsymbol{\epsilon} \cdot \mathbf{k}}{\mathbf{k}^{2}} e^{i \omega_{0} z_{1}} c a_{1} T_{a_{1}} .
\end{aligned}
$$

Note that while the direct $\left\langle M_{1, m, l} M_{1, m^{\prime}, l^{\prime}}^{\dagger}\right\rangle$ ensemble averages lead to non-vanishing modifications to the initial hard spectrum, $\left|M_{0}\right|^{2} \propto|J(p)|^{2} / \mathbf{k}^{2}$, the interference between $M_{0}$ and $M_{1, l, m}$ vanishes because the ensemble average is over a color neutral medium with $\operatorname{Tr} T_{a}(i)=0$.

APPENDIX B: DIAGRAM $M_{2,0,3}=G_{0} X_{1,1} X_{2,1}$

Consider next the gluon two-scatterings amplitude $M_{2,0,3}$ in [26]. Fig. 8 shows that for inclusive processes two interesting cases arise.
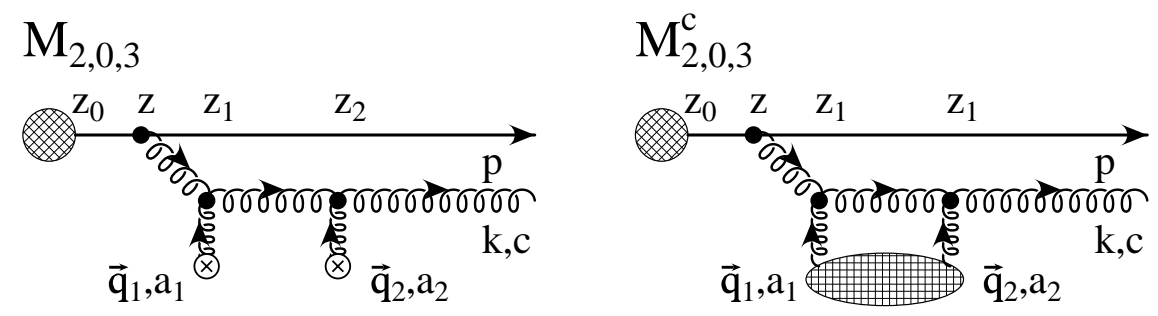

FIG 8. $M_{2,0,3}=G_{0} X_{1,1} X_{2,1}$ "direct" contributes to second order in opacity $\propto\left(\sigma_{e l} / A_{\perp}\right)^{2}$, whereas $M_{2,0,3}^{c}=$ $M_{2,0,3}\left(z_{2}=z_{1}\right) \equiv G_{0} O_{1,1}$ "contact-limit" may contribute to first order in opacity $\propto\left(\sigma_{e l} / A_{\perp}\right)^{1}$ as well.

In the Feynman diagram approach

$$
\begin{aligned}
M_{2,0,3}= & \int \frac{d^{4} q_{1}}{(2 \pi)^{4}} \frac{d^{4} q_{2}}{(2 \pi)^{4}} i J\left(p+k-q_{1}-q_{2}\right) e^{i\left(p+k-q_{1}-q_{2}\right) x_{0}} V\left(q_{1}\right) e^{i q_{1} x_{1}} V\left(q_{2}\right) e^{i q_{2} x_{2}} \times \\
& \times \Lambda_{12}\left(p, k, q_{1}, q_{2}\right) i \Delta\left(p+k-q_{1}-q_{2}\right)(-i) \Delta\left(k-q_{1}-q_{2}\right)(-i) \Delta\left(k-q_{2}\right) \times \\
\approx & J(p+k) e^{i(p+k) x_{0}}\left[\left[c, a_{2}\right], a_{1}\right]\left(T_{a_{2}}(2) T_{a_{1}}(1)\right) \times \\
& \times(-i) \int \frac{d^{2} \mathbf{q}_{1}}{(2 \pi)^{2}}(-i) \int \frac{d^{2} \mathbf{q}_{2}}{(2 \pi)^{2}} 2 i g_{s} \boldsymbol{\epsilon} \cdot\left(\mathbf{k}-\mathbf{q}_{1}-\mathbf{q}_{2}\right) e^{-i \mathbf{q}_{1} \cdot \mathbf{b}_{1}} e^{-i \mathbf{q}_{2} \cdot \mathbf{b}_{2}} \times \\
& \times \int \frac{d q_{1 z}}{(2 \pi)} \frac{d q_{2 z}}{(2 \pi)} \frac{\left(E^{+} k^{+}\right) v\left(\overrightarrow{\mathbf{q}}_{1}\right) v\left(\overrightarrow{\mathbf{q}}_{2}\right) e^{-i q_{1 z}\left(z_{1}-z_{0}\right)} e^{-i q_{2 z}\left(z_{2}-z_{0}\right)}}{\left(\left(p+k-q_{1}-q_{2}\right)^{2}+i \epsilon\right)\left(\left(k-q_{1}-q_{2}\right)^{2}+i \epsilon\right)\left(\left(k-q_{2}\right)^{2}+i \epsilon\right)},
\end{aligned}
$$

where $\mathbf{b}_{i}=\mathbf{x}_{i}-\mathbf{x}_{0}$ are transverse impact parameters, and we used the soft glue and rescattering kinematical simplifications Eqs. (29.34), e.g. $J\left(p+k-q_{1}-q_{2}\right) \approx J(p+k) \approx J(p)$. For the $q_{1 z}$ integral, it is convenient to rewrite the phase as

$$
e^{-i q_{1 z}\left(z_{1}-z_{0}\right)} e^{-i q_{2 z}\left(z_{2}-z_{0}\right)}=e^{-i\left(q_{1 z}+q_{2 z}\right)\left(z_{1}-z_{0}\right)} e^{-i q_{2 z}\left(z_{2}-z_{1}\right)} .
$$


The first longitudinal integral is closely related to Eq. A2

$$
I_{2}\left(p, k, \mathbf{q}_{1}, \overrightarrow{\mathbf{q}}_{2}, z_{1}-z_{0}\right)=\int \frac{d q_{1 z}}{2 \pi} \frac{v\left(q_{1 z}, \mathbf{q}_{1}\right) e^{-i\left(q_{1 z}+q_{2 z}\right)\left(z_{1}-z_{0}\right)}}{\left(\left(p+k-q_{1}-q_{2}\right)^{2}+i \epsilon\right)\left(\left(k-q_{1}-q_{2}\right)^{2}+i \epsilon\right)} .
$$

Since $z_{1}-z_{0} \gg 1 / \mu$, we again close the contour in the lower half $q_{1 z}$ plane and neglect the pole at $-i \mu_{1}$. The remaining $q_{1 z}$ poles are shifted by $-q_{2 z}$ and $\mathbf{q}_{1} \rightarrow \mathbf{q}_{1}+\mathbf{q}_{2}$ relative to Eq. (A3):

$$
\begin{array}{ll}
\bar{q}_{1}=-q_{2 z}+E^{+}+i \epsilon, & \bar{q}_{2}=-q_{2 z}-\omega_{0}-i \epsilon, \\
\bar{q}_{3}=-q_{2 z}+k^{+}-\omega_{(12)}+i \epsilon, & \bar{q}_{4}=-q_{2 z}-\omega_{0}+\omega_{(12)}-i \epsilon,
\end{array}
$$

where now $k^{+} \omega_{(12)}=\left(\mathbf{k}-\mathbf{q}_{1}-\mathbf{q}_{2}\right)^{2}$. The residues at $\bar{q}_{2}, \bar{q}_{4}$ then give

$$
I_{2} \approx i \frac{e^{i \omega_{0}\left(z_{1}-z_{0}\right)}}{E^{+} k^{+} \omega_{(12)}}\left(v\left(-q_{2 z}-\omega_{0}, \mathbf{q}_{1}\right)-v\left(-q_{2 z}-\omega_{0}+\omega_{(12)}, \mathbf{q}_{1}\right) e^{-i \omega_{(12)}\left(z_{1}-z_{0}\right)}\right),
$$

where we have neglected $\mathcal{O}(\exp (-\mu \lambda))$ contributions. This differs from Eq. A6 mainly in that the potential is evaluated near $-q_{2 z}$, which still remains to be integrated over, and $\omega_{1} \rightarrow \omega_{(12)}$.

Next we need the following critical $q_{2 z}$ integral

$$
I_{3}\left(k, \mathbf{q}_{1}, \mathbf{q}_{2}, z_{2}-z_{1}\right) \equiv \int \frac{d q_{2 z}}{2 \pi} \frac{v\left(-q_{2 z}+\delta \omega, \mathbf{q}_{1}\right) v\left(q_{2 z}, \mathbf{q}_{2}\right) e^{-i q_{2 z}\left(z_{2}-z_{1}\right)}}{\left(\left(k-q_{2}\right)^{2}+i \epsilon\right)} .
$$

Fortunately, we are interested in only two extreme limits:

- The limit of well-separated scattering centers $z_{2}-z_{1} \gg 1 / \mu$;

- The special "contact" $z_{2}=z_{1}$ limit to compute unitary contributions.

In the first case, we can proceed ignoring the $q_{2 z}=-i \mu_{1}$ and $-i \mu_{2}$ potential singularities. Then only the pole at $q_{2 z}=\omega_{2}-\omega_{0}-i \epsilon$ contributes and yields

$$
I_{3}\left(k, \mathbf{q}_{1}, \mathbf{q}_{2}, z_{2}-z_{1} \gg 1 / \mu\right) \approx-\frac{i}{k^{+}} v\left(0, \mathbf{q}_{1}\right) v\left(0, \mathbf{q}_{2}\right) e^{-i\left(\omega_{2}-\omega_{0}\right)\left(z_{2}-z_{1}\right)} .
$$

In this case we recover the result quoted in Ref. [27] for $M_{2,0,3}$

$$
\begin{aligned}
M_{2,0,3} \approx & J(p) e^{i p x_{0}}(-i) \int \frac{d^{2} \mathbf{q}_{1}}{(2 \pi)^{2}} v\left(0, \mathbf{q}_{1}\right) e^{-i \mathbf{q}_{1} \cdot \mathbf{b}_{1}}(-i) \int \frac{d^{2} \mathbf{q}_{1}}{(2 \pi)^{2}} v\left(0, \mathbf{q}_{2}\right) e^{-i \mathbf{q}_{2} \cdot \mathbf{b}_{2}} \times \\
& \times\left(2 i g_{s}\right) \frac{\boldsymbol{\epsilon} \cdot\left(\mathbf{k}-\mathbf{q}_{1}-\mathbf{q}_{2}\right)}{\left(\mathbf{k}-\mathbf{q}_{1}-\mathbf{q}_{2}\right)^{2}} e^{i\left(\omega_{0}-\omega_{2}\right) z_{2}} e^{i\left(\omega_{2}-\omega_{(12)}\right) z_{1}}\left(e^{i \omega_{(12)} z_{1}}-e^{i \omega_{(12)} z_{0}}\right) \times \\
& \times\left[\left[c, a_{2}\right], a_{1}\right]\left(T_{a_{2}} T_{a_{1}}\right) .
\end{aligned}
$$

In the general case (including the special contact case with $z_{2}=z_{1}$ ) both $q_{2 z}=-i \mu_{2},-i \mu_{1}$ singularities in the Yukawa potential contribute together with the pole at $q_{2 z}=\omega_{2}-\omega_{0}-i \epsilon$, resulting in

$$
\begin{aligned}
I_{3}\left(k, \mathbf{q}_{1}, \mathbf{q}_{2}, z_{2}-z_{1}\right) \approx & \frac{-i}{k^{+}}\left(v\left(0, \mathbf{q}_{1}\right) v\left(0, \mathbf{q}_{2}\right) e^{-i\left(\omega_{2}-\omega_{0}\right)\left(z_{2}-z_{1}\right)}\right. \\
& \left.\quad-\frac{\left(4 \pi \alpha_{s}\right)^{2}}{2\left(\mu_{1}^{2}-\mu_{2}^{2}\right)}\left(\frac{e^{-\mu_{2}\left(z_{2}-z_{1}\right)}}{\mu_{2}^{2}}-\frac{e^{-\mu_{1}\left(z_{2}-z_{1}\right)} e^{-i \delta \omega\left(z_{2}-z_{1}\right)}}{\mu_{1}^{2}}\right)\right) .
\end{aligned}
$$

For $z_{2}-z_{1}=\lambda \gg 1 / \mu$ this reduces to Eq. B6 . For the special contact contribution $z_{2}-z_{1}=0$ it reduces to

$$
I_{3}\left(k, \mathbf{q}_{1}, \mathbf{q}_{2}, 0\right) \approx \frac{-i}{2 k^{+}} v\left(0, \mathbf{q}_{1}\right) v\left(0, \mathbf{q}_{2}\right),
$$

i.e., exactly $\frac{1}{2}$ of the strength in Eq. (B6). The contact limit of this amplitude is therefore 


$$
\begin{aligned}
M_{2,0,3}^{c} \approx & J(p) e^{i p x_{0}}(-i) \int \frac{d^{2} \mathbf{q}_{1}}{(2 \pi)^{2}} v\left(0, \mathbf{q}_{1}\right) e^{-i \mathbf{q}_{1} \cdot \mathbf{b}_{1}}(-i) \int \frac{d^{2} \mathbf{q}_{2}}{(2 \pi)^{2}} v\left(0, \mathbf{q}_{2}\right) e^{-i \mathbf{q}_{2} \cdot \mathbf{b}_{2}} \times \\
& \times \frac{1}{2}\left(2 i g_{s}\right) \frac{\boldsymbol{\epsilon} \cdot\left(\mathbf{k}-\mathbf{q}_{1}-\mathbf{q}_{2}\right)}{\left(\mathbf{k}-\mathbf{q}_{1}-\mathbf{q}_{2}\right)^{2}} e^{i \omega_{0} z_{1}}\left(1-e^{-i \omega_{(12)}\left(z_{1}-z_{0}\right)}\right)\left[\left[c, a_{2}\right], a_{1}\right]\left(T_{a_{2}} T_{a_{1}}\right) .
\end{aligned}
$$

After this explicit derivation of the factor $\frac{1}{2}$ in the contact limit, we can generalize it to any functional form of the potential as follows. First we assume that a contact interactions only contributes for $\overrightarrow{\mathbf{q}}_{1}=$ $-\overrightarrow{\mathbf{q}}_{2}$, corresponding to no net transverse momentum exchange inside the potential function only, i.e. $v\left(q_{1 z}, \mathbf{q}_{1}\right)=v\left(-q_{2 z},-\mathbf{q}_{2}\right)$. However the distinction has to be kept in the propagators and phases. In this case the potential function $v\left(\overrightarrow{\mathbf{q}}_{1}\right)$, does not appear in the first integral $I_{2}$, Eq. (B2), which is modified to

$$
\begin{aligned}
\bar{I}_{2}\left(p, k, \mathbf{q}_{1}, \overrightarrow{\mathbf{q}}_{2}, z_{1}-z_{0}\right) & =\int \frac{d q_{1 z}}{2 \pi} \frac{e^{-i\left(q_{1 z}+q_{2 z}\right)\left(z_{1}-z_{0}\right)}}{\left(\left(p+k-q_{1}-q_{2}\right)^{2}+i \epsilon\right)\left(\left(k-q_{1}-q_{2}\right)^{2}+i \epsilon\right)} \\
& \approx i \frac{e^{i \omega_{0}\left(z_{1}-z_{0}\right)}}{E^{+} k^{+} \omega_{(12)}}\left(1-e^{-i \omega_{(12)}\left(z_{1}-z_{0}\right)}\right) .
\end{aligned}
$$

This is independent of the functional form of the potential. The second integral, $I_{3}$, then is modified to

$$
\begin{aligned}
\bar{I}_{3}\left(k, \mathbf{q}_{1}=\mathbf{q}_{2}, z_{2}-z_{1}\right) & \equiv \int \frac{d q_{2 z}}{2 \pi} \frac{\left|v\left(q_{2 z}, \mathbf{q}_{2}\right)\right|^{2} e^{-i q_{2 z}\left(z_{2}-z_{1}\right)}}{\left(\left(k-q_{2}\right)^{2}+i \epsilon\right)} \\
& \approx \frac{1}{k^{+}} \int \frac{d q_{2 z}}{2 \pi} \frac{\left|v\left(q_{2 z}, \mathbf{q}_{2}\right)\right|^{2} e^{-i q_{2 z}\left(z_{2}-z_{1}\right)}}{q_{2 z}-\delta \omega+i \epsilon},
\end{aligned}
$$

where $z_{2}-z_{1} \equiv \lambda$ and we have used the finite range of $\left|v\left(q_{z}, q\right)\right|^{2}$, i.e. $v$ is limited within $(-\mu,+\mu)$, to neglect the residue at $q_{2 z} \approx k^{+}$. For large $z_{2}-z_{1}$ compared to the range of $v$, we close below and obtain the dominant residue

$$
\bar{I}_{3} \approx-\frac{i}{k^{+}}\left|v\left(0, \mathbf{q}_{2}\right)\right|^{2} e^{-i\left(z_{2}-z_{1}\right) \delta \omega}
$$

independent approximately of the actual form of $v$ as long as all singularities of $v$ in the lower half plane have imaginary parts $-i \mu_{i}$ with $\mu_{i} \lambda=\mu_{i}\left(z_{2}-z_{1}\right) \gg 1$.

To extract the contact limit, $z_{2}-z_{1}=0$, we can integrate along the real $q_{2 z}$ axis and use the Dirac representation of a pole approaching the real axis:

$$
\frac{1}{q_{2 z}-\delta \omega+i \epsilon}=\frac{q_{2 z}-\delta \omega}{\left(q_{2 z}-\delta \omega\right)^{2}+\epsilon^{2}}-i \pi \delta\left(q_{2 z}-\delta \omega\right)
$$

This gives

$$
k^{+} e^{i \delta \omega\left(z_{2}-z_{1}\right)} \bar{I}_{3} \approx-\frac{i}{2}\left|v\left(\delta \omega, \mathbf{q}_{2}\right)\right|^{2}+\int_{-\infty}^{\infty} \frac{d \tilde{q}_{z}}{2 \pi}\left|v\left(\tilde{q}_{z}+\delta \omega, \mathbf{q}_{2}\right)\right|^{2} \frac{\tilde{q}_{z} \cos \left(\tilde{q}_{z} \lambda\right)-i \tilde{q}_{z} \sin \left(\tilde{q}_{z} \lambda\right)}{\tilde{q}_{z}^{2}+\epsilon^{2}},
$$

where we have introduced $\tilde{q}_{z}=q_{2 z}-\delta \omega$. For our high energy eikonal applications $\delta \omega \ll \mu$, and therefore we can now expand $|v|^{2}$ around $\delta \omega=0$. The correction of $\mathcal{O}(\delta \omega / \mu)$ can be neglected. This leads to a vanishing real part since that part of the integrand is odd. On the other hand, the imaginary part has a finite $\epsilon=0$ limit

$$
-i \int_{-\infty}^{\infty} \frac{d \tilde{q}_{z}}{2 \pi}\left|v\left(\tilde{q}_{z}, \mathbf{q}_{2}\right)\right|^{2} \frac{\sin \left(\tilde{q}_{z} \lambda\right)}{\tilde{q}_{z}}= \begin{cases}0 & \text { if } \mu \lambda=\mu\left(z_{2}-z_{1}\right) \rightarrow 0 \\ -\frac{i}{2}\left|v\left(0, \mathbf{q}_{2}\right)\right|^{2} & \text { if } \mu \lambda=\mu\left(z_{2}-z_{1}\right) \rightarrow \infty\end{cases}
$$

where $\mu$ is the range of the potential $v$ as indicated above. We see that while the detailed interpolation between the asymptotic limits depends on the actual functional form of $v$, the limits Eqs. (B6, B9) and the factor of $\frac{1}{2}$ reduction in the contact $(\lambda=0)$ limit is very general.

We thus confirm that $O_{i, 1}$ diagrams where the two legs are attached to the gluon line can be obtained from the higher order direct diagram by setting $z_{i+1}=z_{i}$ and reducing the strength twice (i.e. times $\frac{1}{2}$ ). 
APPENDIX C: DIAGRAMS $M_{2,0,0}=G_{0} X_{1,0} X_{2,0}$ AND $M_{2,2,0}=G_{0} X_{1,0} G^{-1} X_{2,0} G_{2}$

In those graphs it is the jet rather than the gluon that suffers two sequential scatterings as seen from Fig. 9 .
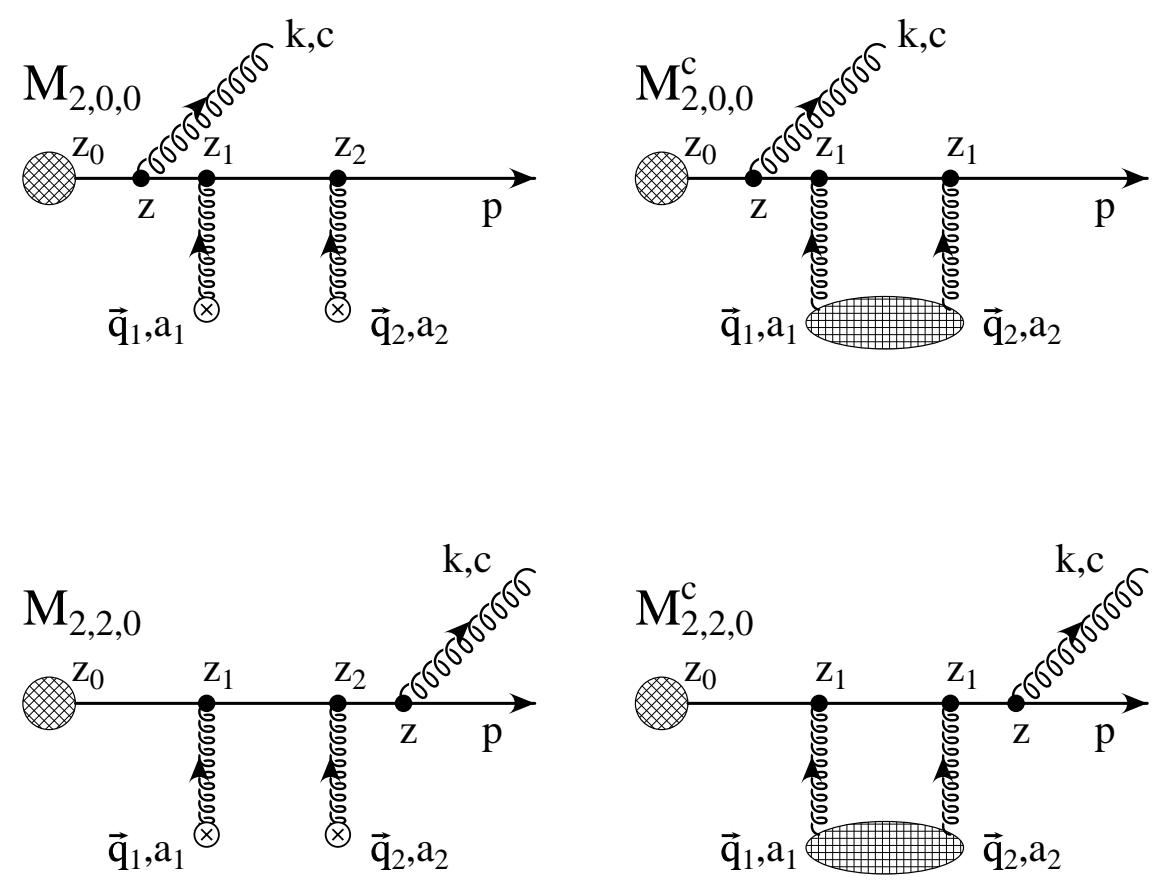

FIG 9. $M_{2,0,0}=G_{0} X_{1,0} X_{2,0}$ and $M_{2,2,0}=G_{0} X_{1,0} G^{-1} X_{2,0} G_{2}$ graphs in the well-separated case together with their $z_{2}=z_{1} \operatorname{limits} M_{2,0,0}^{c} \equiv G_{0} O_{1,0}, M_{2,2,0}^{c} \equiv G_{0} G^{-1} O_{1,0} G_{1}$.

It is straightforward to write the expression for the amplitude

$$
\begin{aligned}
M_{2,0,0}= & \int \frac{d^{4} q_{1}}{(2 \pi)^{4}} \frac{d^{4} q_{2}}{(2 \pi)^{4}} i J\left(p+k-q_{1}-q_{2}\right) e^{i\left(p+k-q_{1}-q_{2}\right) x_{0}} V\left(q_{1}\right) e^{i q_{1} x_{1}} V\left(q_{2}\right) e^{i q_{2} x_{2}} \times \\
& \times\left(-i E^{+}\right)^{2} i g_{s}(2 p+k)_{\mu} \epsilon^{\mu} i \Delta\left(p+k-q_{1}-q_{2}\right) i \Delta\left(p-q_{1}-q_{2}\right) i \Delta\left(p-q_{2}\right) \times \\
& \times a_{2} a_{1} c\left(T_{a_{2}} T_{a_{1}}\right) \\
\approx & J(p) e^{i p x_{0}}(-i) \int \frac{d^{2} \mathbf{q}_{1}}{(2 \pi)^{2}} e^{-i \mathbf{q}_{1} \cdot \mathbf{b}_{1}}(-i) \int \frac{d^{2} \mathbf{q}_{2}}{(2 \pi)^{2}} e^{-i \mathbf{q}_{2} \cdot \mathbf{b}_{2}} \times \\
& \times \frac{2 i g_{s}(\boldsymbol{\epsilon} \cdot \mathbf{k})}{x} e^{i \omega_{0} z_{0}} a_{2} a_{1} c\left(T_{a_{2}} T_{a_{1}}\right)\left(E^{+}\right)^{2} \times \\
& \times \int \frac{d q_{1 z}}{(2 \pi)} \frac{d q_{2 z}}{(2 \pi)} \frac{v\left(\overrightarrow{\mathbf{q}}_{1}\right) v\left(\overrightarrow{\mathbf{q}}_{2}\right) e^{-i q_{1 z}\left(z_{1}-z_{0}\right)} e^{-i q_{2 z}\left(z_{2}-z_{0}\right)}}{\left(\left(p+k-q_{1}-q_{2}\right)^{2}+i \epsilon\right)\left(\left(p-q_{1}-q_{2}\right)^{2}+i \epsilon\right)\left(\left(p-q_{2}\right)^{2}+i \epsilon\right)} .
\end{aligned}
$$

In this case we define

$$
I_{2}\left(p, k, \mathbf{q}_{1}, \overrightarrow{\mathbf{q}}_{2}, z_{1}-z_{0}\right)=\int \frac{d q_{1 z}}{2 \pi} \frac{v\left(q_{1 z}, \mathbf{q}_{1}\right) e^{-i\left(q_{1 z}+q_{2 z}\right)\left(z_{1}-z_{0}\right)}}{\left(\left(p+k-q_{1}-q_{2}\right)^{2}+i \epsilon\right)\left(\left(p-q_{1}-q_{2}\right)^{2}+i \epsilon\right)} . .
$$

Since $z_{1}-z_{0} \gg 1 / \mu$, we neglect the pole at $-i \mu_{1}$. The remaining $q_{1 z}$ poles are

$$
\begin{array}{llrl}
\bar{q}_{1}=-q_{2 z}+E^{+}+k^{+}+i \epsilon, & \bar{q}_{2}=-q_{2 z}-\omega_{0}-i \epsilon, \\
\bar{q}_{3}=-q_{2 z}+E^{+}+i \epsilon, & \bar{q}_{4}=-q_{2 z}-i \epsilon,
\end{array}
$$


where we discarded $\left(\mathbf{p}+\mathbf{k}-\mathbf{q}_{1}-\mathbf{q}_{2}\right)^{2} / E^{+}$relative to $\omega_{0}$. The $\bar{q}_{2}, \bar{q}_{4}$ residues then give

$$
I_{2} \approx-i \frac{v\left(-q_{2 z}, \mathbf{q}_{1}\right)}{\left(E^{+}\right)^{2} \omega_{0}}\left(e^{i \omega_{0}\left(z_{1}-z_{0}\right)}-1\right) .
$$

Not that $\omega_{0}$ has been neglected in the potential relative to $\mu_{1}$. The second integral, $I_{3}$, is then modified to

$$
\begin{aligned}
\bar{I}_{3}\left(p, \mathbf{q}_{1}, \mathbf{q}_{2}, z_{2}-z_{1}\right) & \equiv \int \frac{d q_{2 z}}{(2 \pi)} \frac{v\left(-q_{2 z}, \mathbf{q}_{1}\right) v\left(q_{2 z}, \mathbf{q}_{2}\right) e^{-i q_{2 z}\left(z_{2}-z_{1}\right)}}{\left(\left(p-q_{2}\right)^{2}+i \epsilon\right)} \\
& \approx \frac{i}{E^{+}} v\left(0, \mathbf{q}_{1}\right) v\left(0, \mathbf{q}_{2}\right) \times\left\{\begin{array}{ll}
1 & \text { if } \mu \lambda=\mu\left(z_{2}-z_{1}\right) \rightarrow \infty \\
\frac{1}{2} & \text { if } \mu \lambda=\mu\left(z_{2}-z_{1}\right) \rightarrow 0
\end{array} .\right.
\end{aligned}
$$

Note that $E^{+} \omega_{0}=\mathbf{k}^{2} / x$. With the help of Eqs. (C4.C5) in the case of well-separated scattering centers we obtain

$$
\begin{aligned}
M_{2,0,0}= & J(p) e^{i p x_{0}}(-i) \int \frac{d^{2} \mathbf{q}_{1}}{(2 \pi)^{2}} v\left(0, \mathbf{q}_{1}\right) e^{-i \mathbf{q}_{1} \cdot \mathbf{b}_{1}}(-i) \int \frac{d^{2} \mathbf{q} 2}{(2 \pi)^{2}} v\left(0, \mathbf{q}_{2}\right) e^{-i \mathbf{q}_{2} \cdot \mathbf{b}_{2}} \times \\
& \times \frac{2 i g_{s}(\boldsymbol{\epsilon} \cdot \mathbf{k})}{\mathbf{k}^{2}}\left(e^{i \omega_{0} z_{1}}-e^{i \omega_{0} z_{0}}\right) a_{2} a_{1} c\left(T_{a_{2}} T_{a_{1}}\right) .
\end{aligned}
$$

We thus recover the result from [27]. In the contact limit there is extra factor of $\frac{1}{2}$ relative to the naive expectation and Eq. (C6). Analogous calculation leads to the expected result for $M_{2,2,0}$.

We thus arrive at a conclusion similar to the one in Appendix B, i.e. in the case when the two legs of the virtual contribution are attached to the quark line, $O_{i, 0}$, there is an additional factor of $\frac{1}{2}$ relative to just setting $z_{i+1}=z_{i}$ in the higher order well-separated case amplitudes.

\section{APPENDIX D: DIAGRAMS $M_{2,0,1}=G_{0} X_{1,1} X_{2,0}$ AND $M_{2,0,2}=G_{0} X_{1,0} X_{2,1}$}

In the case when one of the hits is on the parent parton and the other hit is on the radiated gluon we encounter a different situation. Explicit calculation shows this on the example of $M_{2,0,1}^{c}=M_{2,0,1}\left(z_{2}=z_{1}\right)$ in Fig. 10.
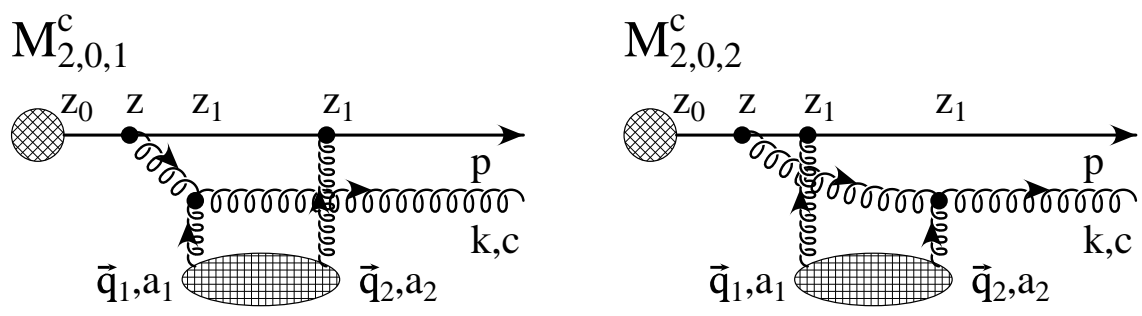

FIG 10. $M_{2,0,1}^{c}=G_{0} O_{1,2}$ and $M_{2,0,2}^{c}=G_{0} O_{1,2}$ topologically indistinct contact diagrams. There are no additional factors of $\frac{1}{2}$ arising from the integration in taking the $z_{2}=z_{1}$ limit in $M_{2,0,1}=G_{0} X_{1,1} X_{2,0}$ and $M_{2,0,2}=G_{0} X_{1,0} X_{2,1}$.

$$
\begin{aligned}
M_{2,0,1}= & \int \frac{d^{4} q_{1}}{(2 \pi)^{4}} \frac{d^{4} q_{2}}{(2 \pi)^{4}} i J\left(p+k-q_{1}-q_{2}\right) e^{i\left(p+k-q_{1}-q_{2}\right) x_{0}} V\left(q_{1}\right) e^{i q_{1} x_{1}} V\left(q_{2}\right) e^{i q_{2} x_{2}} \times \\
& \times\left(-i E^{+}\right) \Lambda_{1} i \Delta\left(p+k-q_{1}-q_{2}\right)(-i) \Delta\left(k-q_{1}\right) i \Delta\left(p-q_{2}\right) \times \\
\approx & J(p) e^{i p x_{0}}(-i) \int \frac{d^{2} \mathbf{q}_{1}}{(2 \pi)^{2}} e^{-i \mathbf{q}_{1} \cdot \mathbf{b}_{1}}(-i) \int \frac{d^{2} \mathbf{q}_{2}}{(2 \pi)^{2}} e^{-i \mathbf{q}_{2} \cdot \mathbf{b}_{2}} \times \\
& \times 2 i g_{s}\left(\boldsymbol{\epsilon} \cdot\left(\mathbf{k}-\mathbf{q}_{1}\right)\right) e^{i \omega_{0} z_{0}} a_{2}\left[c, a_{1}\right]\left(T_{a_{2}} T_{a_{1}}\right)\left(E^{+}\right)^{2} \times
\end{aligned}
$$




$$
\times \int \frac{d q_{1 z}}{(2 \pi)} \frac{d q_{2 z}}{(2 \pi)} \frac{v\left(\overrightarrow{\mathbf{q}}_{1}\right) v\left(\overrightarrow{\mathbf{q}}_{2}\right) e^{-i q_{1 z}\left(z_{1}-z_{0}\right)} e^{-i q_{2 z}\left(z_{2}-z_{0}\right)}}{\left(\left(p+k-q_{1}-q_{2}\right)^{2}+i \epsilon\right)\left(\left(k-q_{1}\right)^{2}+i \epsilon\right)\left(\left(p-q_{2}\right)^{2}+i \epsilon\right)} .
$$

We perform the $q_{1 z}$ integral first

$$
I_{2}\left(p, k, \mathbf{q}_{1}, \overrightarrow{\mathbf{q}}_{2}, z_{1}-z_{0}\right)=\int \frac{d q_{1 z}}{2 \pi} \frac{v\left(q_{1 z}, \mathbf{q}_{1}\right) e^{-i\left(q_{1 z}+q_{2 z}\right)\left(z_{1}-z_{0}\right)}}{\left(\left(p+k-q_{1}-q_{2}\right)^{2}+i \epsilon\right)\left(\left(k-q_{1}\right)^{2}+i \epsilon\right)} .
$$

The pole at $-i \mu_{1}$ is again exponentially suppressed. The poles of interest in the lower half plane are $q_{1 z}=-q_{2 z}-\omega_{0}-i \epsilon$ and $q_{1 z}=-\omega_{0}+\omega_{1}-i \epsilon$. Taking the residues leaves us with

$$
I_{2}=i \frac{e^{i \omega_{0}\left(z_{1}-z_{0}\right)}}{E^{+} k^{+}\left(q_{2 z}+\omega_{1}\right)}\left(v\left(-q_{2 z}-\omega_{0}, \mathbf{q}_{1}\right)-v\left(\omega_{1}-\omega_{0}, \mathbf{q}_{1}\right) e^{-i\left(q_{2 z}+\omega_{1}\right)\left(z_{1}-z_{0}\right)}\right) .
$$

It is important to notice that there is no pole at $q_{2 z}=-\omega_{1}$ in Eq. (D3). The remaining integral over $q_{2 z}$ is

$$
\begin{aligned}
I_{3}\left(p, k, \mathbf{q}_{1}, \mathbf{q}_{2}, z_{1}-z_{0}, z_{2}-z_{1}\right)=\int & \frac{d q_{2 z}}{2 \pi} \frac{1}{q_{2 z}+\omega_{1}}\left(\frac{e^{-i q_{2 z}\left(z_{2}-z_{1}\right)}}{\left(p-q_{2}\right)^{2}+i \epsilon} v\left(-q_{2 z}-\omega_{0}, \mathbf{q}_{1}\right) v\left(q_{2 z}, \mathbf{q}_{2}\right)\right. \\
& \left.-\frac{e^{-i\left(q_{2 z}\left(z_{2}-z_{0}\right)+\omega_{1}\left(z_{1}-z_{0}\right)\right)}}{\left(p-q_{2}\right)^{2}+i \epsilon} v\left(\omega_{1}-\omega_{0}, \mathbf{q}_{1}\right) v\left(q_{2 z}, \mathbf{q}_{2}\right)\right) .
\end{aligned}
$$

Unlike in previous examples, we now show that no factor of $\frac{1}{2}$ arises in the contact limit $z_{2}=z_{1}>z_{0}$. The poles in the lower half plane are $q_{2 z}=-i \epsilon, q_{2 z}=-i \mu_{2}$, and $q_{2 z}=-\omega_{0}-i \mu_{2}$ and the corresponding residues contribute

$$
\begin{aligned}
\operatorname{Res}(-i \epsilon) & \approx \frac{v\left(0, \mathbf{q}_{1}\right) v\left(0, \mathbf{q}_{2}\right)}{E^{+} \omega_{1}}\left(1-e^{-i \omega_{1}\left(z_{1}-z_{0}\right)}\right), \\
\operatorname{Res}\left(-i \mu_{2}\right) & \approx \frac{\left(4 \pi \alpha_{s}\right)^{2} e^{-\mu_{2}\left(z_{2}-z_{1}\right)}}{E^{+} \mu_{2}^{2}\left(2 i \mu_{2}\right)\left(\mu_{1}^{2}-\mu_{2}^{2}-2 i \omega_{0} \mu_{2}\right)}, \\
\operatorname{Res}\left(-i \mu_{1}-\omega_{0}\right) & \approx \frac{\left(4 \pi \alpha_{s}\right)^{2} e^{-\mu_{1}\left(z_{2}-z_{1}\right)} e^{+i \omega_{0}\left(z_{2}-z_{1}\right)}}{E^{+} \mu_{1}^{2}\left(2 i \mu_{1}\right)\left(\mu_{2}^{2}-\mu_{1}^{2}+2 i \omega_{0} \mu_{1}\right)}
\end{aligned}
$$

The exponentially suppressed contributions $\propto \exp \left[-\mu_{2}\left(z_{1}-z_{0}\right)\right]$ in the second and third residues have been neglected, and we made use of the approximation $\omega_{i} \ll \mu_{j}$. In the limit of well separated scattering centers, the second and third residues are also exponentially suppressed. In the contact limit, on the other hand, both the second and third residues in Eq. (D5) contribute. However, impact parameter averaging via Eq. (19) sets $\mu_{1}=\mu_{2}$, and the those two contributions cancel exactly. The result is then

$$
\begin{aligned}
M_{2,0,1}^{c}= & J(p) e^{i p x_{0}}(-i) \int \frac{d^{2} \mathbf{q}_{1}}{(2 \pi)^{2}} e^{-i \mathbf{q}_{1} \cdot \mathbf{b}_{1}} v\left(0, \mathbf{q}_{1}\right)(-i) \int \frac{d^{2} \mathbf{q}_{2}}{(2 \pi)^{2}} e^{-i \mathbf{q}_{2} \cdot \mathbf{b}_{2}} v\left(0, \mathbf{q}_{2}\right) \times \\
& \times 2 i g_{s} \frac{\boldsymbol{\epsilon} \cdot\left(\mathbf{k}-\mathbf{q}_{1}\right)}{\left(\mathbf{k}-\mathbf{q}_{1}\right)^{2}} e^{i\left(\omega_{0}-\omega_{1}\right) z_{1}}\left(e^{i \omega_{1} z_{1}}-e^{i \omega_{1} z_{0}}\right) a_{2}\left[c, a_{1}\right]\left(T_{a_{2}} T_{a_{1}}\right) .
\end{aligned}
$$

We emphasize that in this case, there is no factor of $\frac{1}{2}$ emerging in the $z_{2}=z_{1}$ contact limit $O_{i, 2}$ when one of the legs is attached to the quark line and the other on to the gluon line.

Similarly, the diagram $M_{2,0,2}$ leads to the result quoted in Ref. [27]. In the contact limit, it reduces to $M_{2,0,2}\left(z_{2}=z_{1}\right)=M_{2,0,1}\left(z_{2}=z_{1}\right)$. However, it is important to point out that in the contact limit only one of the diagrams must be taken into account in order to avoid over counting. This can be directly seen from the expansion in the powers of the interaction Lagrangian. Alternatively, we get the correct contact answer by taking the limit of both cross contact diagrams and multiplying each by $\frac{1}{2}$. 


\section{APPENDIX E: ZERO MEASURE CONTACT LIMIT OF \\ $M_{2,1,0}=X_{1,0} G_{1} X_{2,0}$ AND $M_{2,1,1}=X_{1,0} G_{1} X_{2,1}$}

In calculating the different contributions coming from two interactions with the same potential centered around $\overrightarrow{\mathbf{x}}_{1}$ we have to take into account the two graphs given in Fig. 11, where one of the hits occurs before the gluon emission vertex and the other one after.
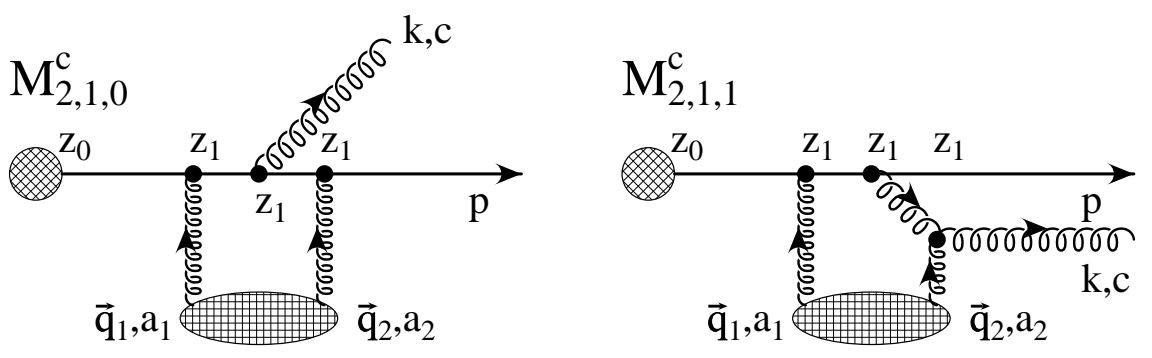

FIG 11. Diagrams $M_{2,1,0}^{c}=X_{1,0} G_{1} X_{2,0}\left(z_{2}=z_{1}\right)$ and $M_{2,1,1}^{c} X_{1,0} G_{1} X_{2,1}\left(z_{2}=z_{1}\right)$ of $\mathcal{O}(0)$ according to the time-ordered perturbation theory.

In the framework of time-ordered perturbation theory from [27, 26] the graphs are identically zero in the contact limit because $\int_{t_{1}}^{t_{1}} d t \cdots \equiv 0$. We here present a more detailed study of the validity of this argument. In the contact limit $M_{2,1,0} \rightarrow M_{2,1,0}^{c}=X_{1,0} G_{1} X_{1,0}$. We will show that this contribution vanishes in the small $x=k^{+} / E^{+}$limit

$$
\begin{aligned}
M_{2,1,0}^{c}= & \int \frac{d^{4} q_{1}}{(2 \pi)^{4}} \frac{d^{4} q_{2}}{(2 \pi)^{4}} i J\left(p+k-q_{1}-q_{2}\right) e^{i\left(p+k-q_{1}-q_{2}\right) x_{0}} V\left(q_{1}\right) e^{i q_{1} x_{1}} V\left(q_{2}\right) e^{i q_{2} x_{1}} \times \\
& \times\left(-i E^{+}\right)^{2} i g_{s}\left(2\left(p-q_{2}\right)+k\right)_{\mu} \epsilon^{\mu} i \Delta\left(p+k-q_{1}-q_{2}\right) i \Delta\left(p+k-q_{2}\right) i \Delta\left(p-q_{2}\right) \times \\
& \times a_{2} c a_{1}\left(T_{a_{2}} T_{a_{1}}\right) \\
\approx & J(p) e^{i p x_{0}}(-i) \int \frac{d^{2} \mathbf{q}_{1}}{(2 \pi)^{2}} e^{-i \mathbf{q}_{1} \cdot \mathbf{b}_{1}}(-i) \int \frac{d^{2} \mathbf{q}_{2}}{(2 \pi)^{2}} e^{-i \mathbf{q}_{2} \cdot \mathbf{b}_{1}} \times \\
& \times 2 i g_{s} \frac{\boldsymbol{\epsilon} \cdot \mathbf{k}}{\mathbf{k}^{2}} e^{i \omega_{0} z_{0}} a_{2} c a_{1}\left(T_{a_{2}} T_{a_{1}}\right)\left(E^{+}\right)^{2} \int \frac{d q_{1 z}}{(2 \pi)} \frac{d q_{2 z}}{(2 \pi)} v\left(\overrightarrow{\mathbf{q}}_{1}\right) v\left(\overrightarrow{\mathbf{q}}_{2}\right) \times \\
& \times \frac{e^{-i\left(q_{1 z}+q_{2 z}\right)\left(z_{1}-z_{0}\right)}}{\left(p+k-q_{1}-q_{2}\right)^{2}+i \epsilon}\left(\frac{1}{\left(p-q_{2}\right)^{2}+i \epsilon}-\frac{1}{\left(p+k-q_{2}\right)^{2}+i \epsilon}\right) .
\end{aligned}
$$

In writing this we have made use the simplifying soft gluon and weak interaction approximations in Eq. (29).

We can now perform the $q_{1 z}$ integral, which brings a factor of $1 / E^{+}$and sets the overall phase to 0 . We have to now perform

$$
I_{3}=\int \frac{q_{2 z}}{2 \pi} v\left(-q_{2 z}, \mathbf{q}_{1}\right) v\left(q_{2 z}, \mathbf{q}_{2}\right)\left(\frac{1}{\left(p-q_{2}\right)^{2}+i \epsilon}-\frac{1}{\left(p+k-q_{2}\right)^{2}+i \epsilon}\right) .
$$

We close in the upper half plane where there are four poles $q_{2 z}=i \mu_{1}, q_{2 z}=i \mu_{2}, q_{2 z}=E^{+}+i \epsilon, q_{2 z}=$ $E^{+}+k^{+}+i \epsilon$. The residues at the poles involving $E^{+}$are suppressed by a factor $\left(1 / E^{+}\right)^{4}$ and have negligible contribution in the high energy approximation. The remaining two residues give

$$
\begin{aligned}
& \operatorname{Res}\left(i \mu_{1}\right) \approx \frac{\left(4 \pi \alpha_{s}\right)^{2}}{E^{+}\left(2 \mu_{1}^{2}\right)\left(\mu_{2}^{2}-\mu_{1}^{2}\right)}\left(-\frac{k^{+}}{E^{+}}\right), \\
& \operatorname{Res}\left(i \mu_{2}\right) \approx \frac{\left(4 \pi \alpha_{s}\right)^{2}}{E^{+}\left(2 \mu_{2}^{2}\right)\left(\mu_{1}^{2}-\mu_{2}^{2}\right)}\left(-\frac{k^{+}}{E^{+}}\right) .
\end{aligned}
$$


We have discarded $\omega_{0}$ relative to $\mu_{i}$ and carried the $k^{+} / E^{+}$expansion to first order. It is important to notice the sum of the residues is not singular for the special case of interest when the contact limit contribution is averaged over the transverse position of the scatterer, i.e. $\mu_{1}=\mu_{2}$. The above contribution is suppressed by an $\mathcal{O}\left(k^{+} / E^{+}\right)$factor relative to the other graphs. In the high energy $E^{+} \rightarrow \infty$ limit, where the time-ordered perturbation theory works, $M_{2,1,0}\left(z_{2}=z_{1}\right) \approx 0$. Similar calculation for $M_{2,1,1} \rightarrow M_{2,1,1}^{c} X_{1,0} G_{1} X_{1,1}$ shows suppression of the order $\mathcal{O}\left(\mu / k^{+}\right)$and is approximately zero according to the $k^{+} \gg \mu$ regime (28) in which the problem is set up.

This leads us to the conclusion that the naive "zero measure" argument for such graphs is approximately valid for our kinematics.

\section{APPENDIX F: AMPLITUDE ITERATION TECHNIQUE TO SECOND ORDER}

To illustrate the general iteration procedure and check the general iteration results using the reaction operator approach, we construct the classes for rank 1 and 2 explicitly.

For the two rank 1 classes, we apply the operators $\hat{D}_{1}$ and $\hat{V}_{1}$ on the hard vertex amplitude Eq. (39) once to obtain

$$
\begin{aligned}
G_{0} D_{1} & =-\mathbf{H} e^{i \omega_{0} z_{0}} a_{1} c-\mathbf{C}_{1} e^{i\left(\omega_{0} z_{1}-\omega_{1} z_{10}\right)}\left[c, a_{1}\right]-\mathbf{B}_{1} e^{i \omega_{0} z_{1}}\left[c, a_{1}\right], \\
G_{0} V_{1} & =\frac{C_{R}+C_{A}}{2} \mathbf{H} e^{i \omega_{0} z_{0}} c-\frac{C_{A}}{2} \mathbf{C}_{1} e^{i\left(\omega_{0} z_{1}-\omega_{1} z_{10}\right)} c-\frac{C_{A}}{2} \mathbf{B}_{1} e^{i \omega_{0} z_{1}} c .
\end{aligned}
$$

We recall that $z_{i j} \equiv z_{i}-z_{j}, \Delta z_{i} \equiv z_{i}-z_{i-1}$. These are all amplitudes needed for the first order in opacity $L / \lambda$ calculation.

Some of the rank two classes are obtained from the rank 1 classes Eq. (F2) through relabeling, i.e. $G_{0} D_{2} \equiv G_{0} D_{1}(1 \rightarrow 2), G_{0} V_{2} \equiv G_{0} V_{1}(1 \rightarrow 2)$. The rest are readily derived from Eqs. ([F1, F2 $)$ through our iteration scheme Eqs. (76, 82)

$$
\begin{aligned}
G_{0} D_{1} D_{2}= & -\mathbf{H} e^{i \omega_{0} z_{0}} a_{2} a_{1} c-\mathbf{C}_{1} e^{i\left(\omega_{0} z_{1}-\omega_{1} z_{10}\right)} a_{2}\left[c, a_{1}\right]-\mathbf{C}_{2} e^{i\left(\omega_{0} z_{2}-\omega_{2} z_{20}\right)} a_{1}\left[c, a_{2}\right] \\
& -\mathbf{B}_{1} e^{i \omega_{0} z_{1}} a_{2}\left[c, a_{1}\right]-\mathbf{B}_{2} e^{i \omega_{0} z_{2}}\left[c, a_{2}\right] a_{1} \\
& -\mathbf{C}_{(12)} e^{i\left(\omega_{0} z_{2}-\omega_{2} z_{21}-\omega_{(12)} z_{10}\right)}\left[\left[c, a_{2}\right], a_{1}\right]-\mathbf{B}_{2(12)} e^{i\left(\omega_{0} z_{2}-\omega_{2} z_{21}\right)}\left[\left[c, a_{2}\right], a_{1}\right] \\
G_{0} D_{1} V_{2}= & \frac{C_{R}+C_{A}}{2} \mathbf{H} e^{i \omega_{0} z_{0}} a_{1} c \\
& +\frac{C_{R}+C_{A}}{2} \mathbf{C}_{1} e^{i\left(\omega_{0} z_{1}-\omega_{1} z_{10}\right)}\left[c, a_{1}\right]+\mathbf{C}_{2} e^{i\left(\omega_{0} z_{2}-\omega_{2} z_{20}\right)} a_{2} a_{1}\left[c, a_{2}\right] \\
& +\frac{C_{R}+C_{A}}{2} \mathbf{B}_{1} e^{i \omega_{0} z_{1}}\left[c, a_{1}\right]-\frac{C_{A}}{2} \mathbf{B}_{2} e^{i \omega_{0} z_{2}} c a_{1} \\
& +\mathbf{C}_{(12)} e^{i\left(\omega_{0} z_{2}-\omega_{2} z_{21}-\omega_{(12)} z_{10}\right)} a_{2}\left[\left[c, a_{2}\right], a_{1}\right]+\mathbf{B}_{2(12)} e^{i\left(\omega_{0} z_{2}-\omega_{2} z_{21}\right)} a_{2}\left[\left[c, a_{2}\right], a_{1}\right] \\
G_{0} V_{1} D_{2}= & \frac{C_{R}+C_{A}}{2} \mathbf{H} e^{i \omega_{0} z_{0}} a_{2} c \\
& -\frac{C_{A}}{2} \mathbf{C}_{1} e^{i\left(\omega_{0} z_{1}-\omega_{1} z_{10}\right)} a_{2} c+\frac{C_{R}+C_{A}}{2} \mathbf{C}_{2} e^{i\left(\omega_{0} z_{2}-\omega_{2} z_{20}\right)}\left[c, a_{2}\right] \\
& -\frac{C_{A}}{2} \mathbf{B}_{1} e^{i \omega_{0} z_{1}} a_{2} c+\frac{C_{R}}{2} \mathbf{B}_{2} e^{i \omega_{0} z_{2}}\left[c, a_{2}\right] \\
& -\frac{C_{A}}{2} \mathbf{C}_{(12)} e^{i\left(\omega_{0} z_{2}-\omega_{2} z_{21}-\omega_{(12)} z_{10}\right)}\left[c, a_{2}\right]-\frac{C_{A}}{2} \mathbf{B}_{2(12)} e^{i\left(\omega_{0} z_{2}-\omega_{2} z_{21}\right)}\left[c, a_{2}\right] \\
G_{0} V_{1} V_{2}= & -\frac{\left(C_{R}+C_{A}\right)^{2}}{4} \mathbf{H} e^{i \omega_{0} z_{0}} c
\end{aligned}
$$




$$
\begin{aligned}
& +\frac{C_{A}\left(C_{A}+C_{R}\right)}{4} \mathbf{C}_{1} e^{i\left(\omega_{0} z_{1}-\omega_{1} z_{10}\right)} c+\frac{C_{A}\left(C_{R}+C_{A}\right)}{4} \mathbf{C}_{2} e^{i\left(\omega_{0} z_{2}-\omega_{2} z_{20}\right)} c \\
& +\frac{C_{A}\left(C_{R}+C_{A}\right)}{4} \mathbf{B}_{1} e^{i \omega_{0} z_{1}} c+\frac{C_{R} C_{A}}{4} \mathbf{B}_{2} e^{i \omega_{0} z_{2}} c \\
& -\frac{C_{A}^{2}}{4} \mathbf{C}_{(12)} e^{i\left(\omega_{0} z_{2}-\omega_{2} z_{21}-\omega_{(12)} z_{10}\right)} c-\frac{C_{A}^{2}}{4} \mathbf{B}_{2(12)} e^{i\left(\omega_{0} z_{2}-\omega_{2} z_{21}\right)} c .
\end{aligned}
$$

With this explicit construction of the relevant classes, we can compute the differential gluon probability up to second order in the opacity expansion. Similar calculations can be performed for the Gunion-Bertsch case as well.

To first order in opacity $L / \lambda$ the probability to radiate a gluon from either quark or gluon jets is proportional to

$$
\begin{gathered}
\frac{1}{d_{R}}\left\langle\operatorname{Tr}\left[G_{0} D_{1} D_{1}^{\dagger} G_{0}^{\dagger}+2 \operatorname{Re}\left(G_{0} V_{1} G_{0}^{\dagger}\right)\right]\right\rangle_{v}=C_{R} C_{A}\left\langle\left[\left(-2 \mathbf{C}_{1} \cdot \mathbf{B}_{1}\right)\left(1-\cos \left(\omega_{1} \Delta z_{1}\right)\right)\right]\right\rangle_{v} \\
=C_{R} C_{A} \int d \mathbf{q}_{1}\left(\bar{v}^{2}\left(\mathbf{q}_{1}\right)-\delta^{2}\left(\mathbf{q}_{1}\right)\right)\left[\left(-2 \mathbf{C}_{1} \cdot \mathbf{B}_{1}\right)\left(1-\cos \left(\omega_{1} \Delta z_{1}\right)\right)\right],
\end{gathered}
$$

where the 17 terms in Eqs. $(43,44)$ collapse to a single term with color trivial factor $C_{R} C_{A} d_{R}$ interpretable as gluon final state interaction only. We note that if the normalized potential $\bar{v}^{2}\left(\mathbf{q}_{1}\right)$ is replaced by $\bar{v}^{2}\left(\mathbf{q}_{1}\right)-$ $\delta^{2}\left(\mathbf{q}_{1}\right)$ the result of Eq. (F7) remains unchanged.

To second order in opacity we find that

$$
\begin{aligned}
& \frac{1}{d_{R}}\left\langle\operatorname { T r } \left[ G_{0} D_{1} D_{2} D_{2}^{\dagger} D_{1}^{\dagger} G_{0}^{\dagger}+\left(G_{0} V_{1} D_{2} D_{2}^{\dagger} G_{0}^{\dagger}+\text { h.c. }\right)+\left(G_{0} D_{1} V_{2} D_{1}^{\dagger} G_{0}^{\dagger}+\text { h.c. }\right)\right.\right. \\
& \left.\left.+\left(G_{0} V_{1} V_{2} G_{0}^{\dagger}+\text { h.c. }\right)+\left(G_{0} V_{1} V_{2}^{\dagger} G_{0}^{\dagger}+\text { h.c. }\right)\right]\right\rangle_{v} \\
& =C_{R} C_{A}^{2}\left\langle\left[ 2 \mathbf{C}_{1} \cdot \mathbf{B}_{1}\left(1-\cos \left(\omega_{1} \Delta z_{1}\right)\right)+2 \mathbf{C}_{2} \cdot \mathbf{B}_{2}\left(\cos \left(\omega_{2} \Delta z_{2}\right)-\cos \left(\omega_{2}\left(\Delta z_{1}+\Delta z_{2}\right)\right)\right.\right.\right. \\
& -2 \mathbf{C}_{(12)} \cdot \mathbf{B}_{2(12)}\left(1-\cos \left(\omega_{(12)} \Delta z_{1}\right)\right) \\
& \left.\left.-2 \mathbf{C}_{(12)} \cdot \mathbf{B}_{2}\left(\cos \left(\omega_{2} \Delta z_{2}\right)-\cos \left(\omega_{(12)} \Delta z_{1}+\omega_{2} \Delta z_{2}\right)\right)\right]\right\rangle_{v} \\
& =C_{R} C_{A}^{2} \prod_{i=1,2} \int d \mathbf{q}_{i}\left(\bar{v}^{2}\left(\mathbf{q}_{i}\right)-\delta^{2}\left(\mathbf{q}_{i}\right)\right)\left[-2 \mathbf{C}_{(12)} \cdot \mathbf{B}_{2(12)}\left(1-\cos \left(\omega_{(12)} \Delta z_{1}\right)\right)\right. \\
& \left.-2 \mathbf{C}_{(12)} \cdot \mathbf{B}_{2}\left(\cos \left(\omega_{2} \Delta z_{2}\right)-\cos \left(\omega_{(12)} \Delta z_{1}+\omega_{2} \Delta z_{2}\right)\right)\right] .
\end{aligned}
$$

Eqs. ( $\mathrm{F7}, \mathrm{F} 8)$ have simple physical interpretation. We showed that for both quark and gluon jets a simple color trace $\propto C_{A}^{n}$ survives. In general this can be seen from Eqs. (76, 82, 81). Thus the distribution of the radiated gluons is interpretable as the interference of the Cascade amplitude that has the knowledge of all final state interactions with the effective color currents, i.e. the Gunion-Bertsch amplitudes, originating at the scattering centers and also having all possible subsequent interactions with the gluon line. The formation physics effects arise from phase differences that store the information on the cumulative formation lengths before the effective color current emission. The averaging over the momentum transfers is seen to be effectively over a modified potential that vanishes on integration.

[1] J. D. Bjorken, FERMILAB-PUB-82-59-THY unpublished.

[2] M. Gyulassy and M. Plümer, Phys. Lett. B 243 (1990) 432.

[3] M. H. Thoma and M. Gyulassy, Nucl. Phys. B 351 (1991) 491.

[4] M. Gyulassy, M. Plümer, M.H. Thoma and X.-N. Wang, Nucl. Phys. A 538 (1992) 37c. 
[5] X.-N. Wang and M. Gyulassy, Phys. Rev. Lett. 68 (1992) 1480.

[6] M. Gyulassy and X.-N. Wang, Nucl. Phys. B 420 (1994) 583.

[7] X. Wang, M. Gyulassy and M. Plumer, Phys. Rev. D51, 3436 (1995) hep-ph/9408344].

[8] X. Wang, Z. Huang and I. Sarcevic, Phys. Rev. Lett. 77, 231 (1996) hep-ph/9605213.

[9] X. Wang, Phys. Rept. 280, 287 (1997) hep-ph/9605214.

[10] R. Baier, Yu.L. Dokshitzer, A.H. Mueller, S. Peigné and D. Schiff, Nucl. Phys. B 483 (1997) 291; [hep$\mathrm{ph} / 9607355$.

[11] R. Baier, Yu.L. Dokshitzer, A.H. Mueller, S. Peigné and D. Schiff, Nucl. Phys. B 484 (1997) 265.

[12] R. Baier, Yu.L. Dokshitzer, A.H. Mueller and D. Schiff, Nucl. Phys. B 531 (1998) 403.

[13] B.G. Zhakharov, JETP Letters 63 (1996) 952.

[14] B.G. Zhakharov, JETP Letters 65 (1997) 615.

[15] I. P. Lokhtin and A. M. Snigirev, Phys. Lett. B440, 163 (1998).

[16] R. Baier, Y. L. Dokshitzer, A. H. Mueller and D. Schiff, Phys. Rev. C60, 064902 (1999)

[17] I. P. Lokhtin and A. M. Snigirev, hep-ph/0004176.

[18] B. Z. Kopeliovich, A. V. Tarasov and A. Schafer, Phys. Rev. C59, 1609 (1999) hep-ph/9808378.

[19] U.A. Wiedemann, M. Gyulassy, Nucl. Phys. B 560 (1999) 345; hep-ph/9906257.

[20] U. A. Wiedemann, hep-ph/0003021.

[21] X. Wang, Phys. Rev. C61, 064910 (2000) nucl-th/9812021.

[22] X. Guo and X. Wang, hep-ph/0005044.

[23] S. Bass et al., Nucl. Phys. A 661 (1999) 205c.

[24] X.-N. Wang, Phys. Rev. Lett. 81 (1998) 2655.

[25] M. Gyulassy and P. Levai, Phys. Lett. B 442, (1998) 1.

[26] M. Gyulassy, P. Lévai, I. Vitev, Nucl. Phys. A 661 (1999) 637c.

[27] M. Gyulassy, P. Lévai, I. Vitev, Nucl. Phys. B 571 (2000) 197.

[28] M. Gyulassy, P. Lévai, I. Vitev, CU-TP-976; nucl-th/0005032.

[29] K.J. Eskola, K. Kajantie, P.V. Ruuskanen, K. Tuominen, Nucl. Phys. B 570 (2000) 379.

[30] L.D. Landau and I.J. Pomeranchuk, Dolk. Akad. Nauk. SSSR 92 (1953) 92.

[31] A.B. Migdal, Phys. Rev. 103 (1956) 1811.

[32] J.F. Gunion and G. Bertsch, Phys. Rev. D 25 (1982) 746.

[33] R. Field, Applications of Perturbative QCD, Addison-Wesley, 1989.

[34] U. A. Wiedemann, hep-ph/0005129.

[35] B. Zhang, Comput. Phys. Commun. 109, 193 (1998).

[36] M. Gyulassy, Y. Pang and B. Zhang, Nucl. Phys. A626, (1997) 999.

[37] D. Molnar and M. Gyulassy, nucl-th/0005051.

[38] D. Molnár, MPC0.1.2., code available at site http://nt3.phys.columbia.edu/people/molnard.

[39] A.C. Genz and A.A. Malik, J. Comput. Appl. Math. 6 (1980) 295.

[40] A. van Doren and L. de Ridder, J. Comput. Appl. Math. 2 (1976) 207. 